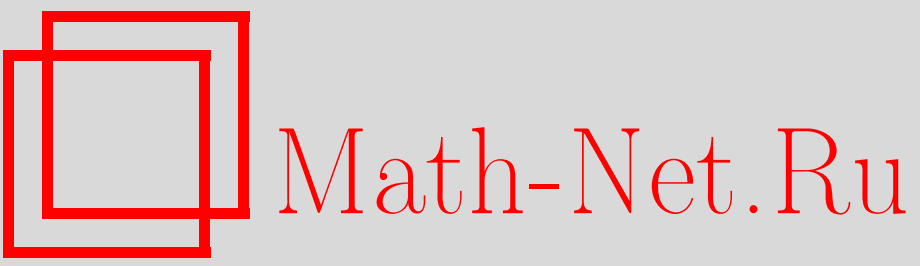

А. В. Маршаков, Струны, суперсимметричные калибровочные теории и интегрируемые системы, ТМФ, 1999, том 121, номер 2, 179-243

DOI: https://doi.org/10.4213/tmf804

Использование Общероссийского математического портала Math-Net.Ru подразумевает, что вы прочитали и согласны с пользовательским соглашением

http://www.mathnet.ru/rus/agreement

Параметры загрузки:

IP: 3.80 .181 .102

26 апреля 2023 г., 14:17:06

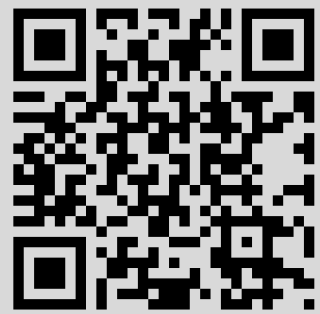




\section{СТРУНЫ, СУПЕРСИММЕТРИЧНЫЕ КАЛИБРОВОЧНЫЕ ТЕОРИИ И ИНТЕГРИРУЕМЫЕ СИСТЕМЫ ${ }^{1)}$}

Рассматриваются суперсимметричные калибровочные теории Янга-Миллса в рамках непертурбативной теории струн или М-теории и обсуждаются компактификации, отвечающие хорошо известным точным эффективным теориям Виттена-Зайберга. Подробно обсуждаются возникающие при этом параллели с формулировкой теории Виттена-Зайберга в терминах интегрируемых систем и демонстрируется, как связь с интегрируемыми системами возникает в контексте теории струн.

\section{СОДЕРЖАНИЕ}

1. Введение. Суперсимметричные калибровочные теории

1.1. Суперсимметричные калибровочные теории как вакуумы теории

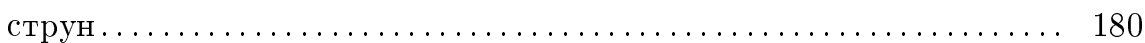

1.2. Струны, М-теория, компактификации и D-браны . . . . . . . . . . . . 182

1.3. Теория струн и системы Янга-Миллса-Хиггса . . . . . . . . . . . . . . 185

1.4. Эффективная теория Виттена-Зайберга . . . . . . . . . . . . . . . . 188

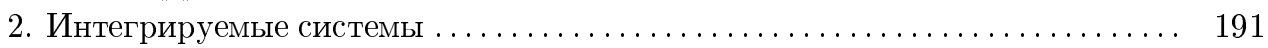

2.1. Конечнозонные решения: конструкция Кричевера . . . . . . . . . . . . 191

2.2. Системы Хитчина . . . . . . . . . . . . . . . . . . . . . . . . . 195

2.3. Деформации конечнозонных решений и тау-функция иерархии Уизема 198

3. Струны и теория Виттена-Зайберга . . . . . . . . . . . . . . . . . . 203

3.1. Комплексные кривые как часть геометрии пространства-времени . . . 203

3.2. D-браны и интегрируемые системы . . . . . . . . . . . . . . . . . . . 204

3.3. $\mathcal{N}=1$ спектральные кривые и солитоны . . . . . . . . . . . . . 208

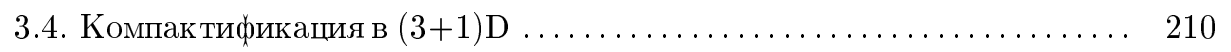

4. Интегрируемые уравнения в топологических струнных моделях и теории

Виттена-Зайберга ................................. 212

\footnotetext{
${ }^{1)}$ Статья написана по заказу Редколлегии.

${ }^{*}$ Физический институт им. П.Н. Лебедева РАН, Институт теоретической и экспериментальной физики, Москва, Россия. E-mail: mars@lpi.ac.ru, andrei@heron.itep.ru, marshakov@nbivms.nbi.dk
} 
4.1. Топологические теории: препотенциал и уравнения Виттена-Дийк-

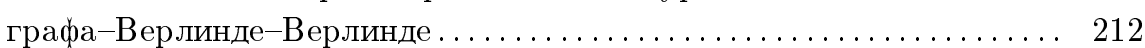

4.2. Топологические теории струн и $2 \mathrm{D}$ квантовая гравитация . . . . . . . 220

4.3. Теория Виттена-Зайберга, иерархии Уизема и уравнения ренормгруп-

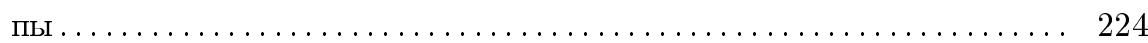

5. Заключение......................................... 240

\section{1. ВВЕДЕНИЕ. СУПЕРСИММЕТРИЧНЫЕ КАЛИБРОВОЧНЫЕ ТЕОРИИ}

Калибровочные теории, или теории безмассовых векторных полей описывают все взаимодействия, кроме гравитационного. Более того, если рассматривать калибровочные поля как безмассовые возбуждения открытых струн, то даже гравитационное взаимодействие нельзя считать вполне независимым. Общий подход к теории калибровочных полей как “производной” от теории струн ведет к ее более глубокому пониманию и уже позволил получить множество замечательных новых результатов.

В частности, в последние годы наметился серьезный прогресс в понимании структуры суперсимметричных калибровочных теорий на больших расстояниях или при малых энергиях. Эта структура существенно непертурбативна и не может быть получена традиционными методами квантовой теории поля. Оказывается, однако, что с точки зрения непертурбативной теории струн (или на современном языке М-теории) по крайней мере некоторые непертурбативные свойства калибровочных теорий становятся более или менее объяснимыми. Более того, связь непертурбативных суперсимметричHux теорий струн и калибровочных теорий основана на тех же принципах, что и связь пространств модулей калибровочных теорий с комплексной геометрией и теорией интегрируемых систем. Тем самым струнное происхождение теории полей Янга-Миллса оказывается ключевым местом в проблеме понимания до сих пор загадочной связи низкоэнергетических суперсимметричных калибровочных теорий с интегрируемыми системами.

Цель данной работы - обсудить именно эти проблемы. Мы начнем с обшего введения в суперсимметричную калибровочную теорию, рассматриваемую как низкоэнергетический предел теории струн. Затем в разделе 2 приведем некоторые полезные сведения из теории интегрируемых систем. В разделе 3 мы обратимся непосредственно к проблеме построения низкоэнергетической структуры суперсимметричных калибровочных теорий из геометрических представлений М-теории и покажем, как на этом пути возникают хорошо знакомые интегрируемые системы. Наконец, в разделе 4 мы обсудим непосредственно интегрируемые уравнения, возникающие в контексте теории Виттена-Зайберга, и продемонстрируем их сходство с интегрируемыми структурами двумерных топологических моделей.

1.1. Суперсимметричные калибровочные теории как вакуумы теории струн. Мы начнем с того давно известного наблюдения теории струн, что теория одномерных протяженных объектов - (открытых) струн - естественным образом содержит векторные поля. Квантовая механика струн и требование репараметризационной инва- 
риантности относительно замен “внутренних" струнных координат приводят к безмассовости векторных полей (массы других полей спектра струны измеряются в единицах массы Планка или струнной длины $\sqrt{\alpha^{\prime}}$ ), т.е. к появлению калибровочных теорий в полевом пределе теории струн $\alpha^{\prime} \rightarrow 0$, когда масса остальных полей становится бесконечной и их возбуждения можно не учитьвать. Спектр струны содержит также тахион, с которым связаны дополнительные трудности; наиболее эффективным инструментом их преодоления является введение суперсимметрии (подробнее см. книгу [1] и приведенную в ней библиографию). Векторное поле $A_{\mu}(x)$ легко превращается в неабелево, если приписать концам струны индексы фундаментального (кварк-антикваркового) представления; при этом поле становится матрицей $\left\|\mathbf{A}^{i j}\right\|$ в присоединенном представлении соответствуюшей калибровочной группы (рис. 1). В суперсимметричной теории струн безмассовый сектор содержит векторные супермультиплеты, в которых остальные поля достраиваются по суперсимметрии; в низкоэнергетическом пределе это приводит к появлению суперсимметричной теории полей Янга-Миллса как возможного вакуума теории струн. Согласно современным общим представлениям, квантовые теории поля (в частности, суперсимметричные калибровочные теории) могут рассматриваться как различные вакуумы теории струн. Эти вакуумы могут быть связаны друг с другом преобразованиями дуальности - дискретными преобразованиями, переводящими друг в друга различные вакуумы теории струн, а следовательно, и различные квантовые теории поля.

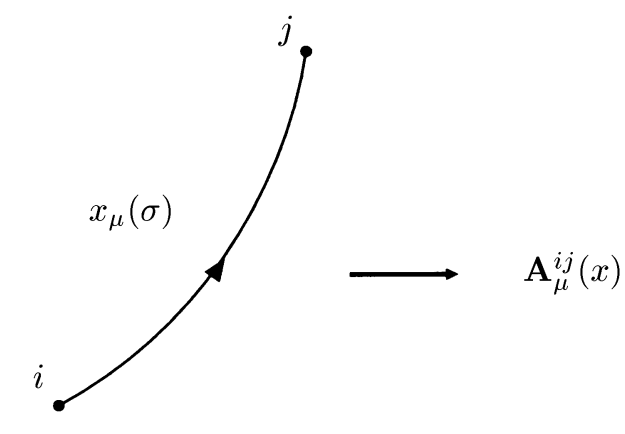

Рис. 1. Безмассовое неабелево векторное поле как открытая струна с кварками на концах.

Основным примером дуальности является симметрия в теории струн в пространстве-времени с компактными направлениями (на окружности) $\varphi \sim \varphi+2 \pi R n$, где $n \in \mathbb{Z}-$ любое целое число. Спектр такой теории и однопетлевая статсумма инвариантны относительно преобразования дуальности $R \leftrightarrow \alpha^{\prime} / R[2]$. Эта инвариантность является следствием того, что наряду со стандартным дискретным спектром частиц на окружности $p \sim n / R, n \in \mathbb{Z}$, имеющимся, естественно, в обычной квантовой теории поля с компактными измерениями, существует другой тип чисто струнных возбуждений: струна может "намотаться" на окружность, дав при этом вклад в спектр $p \sim m R / \alpha^{\prime}, m \in \mathbb{Z}$. В пределе "декомпактификации" $R \rightarrow \infty$, первая часть спектра станет непрерывной 
(как и в обычной квантовой теории поля), в то время как струнные возбуждения становятся бесконечно тяжелыми, их вклад в статсумму - пренебрежимо малым. Однако полный спектр

$$
E_{n, m}=\left(\frac{n}{R}\right)^{2}+\left(\frac{m R}{\alpha^{\prime}}\right)^{2} \quad \forall n, m
$$

очевидно инвариантен относительно замены $R \leftrightarrow \alpha^{\prime} / R$.

Теперь, если рассмотреть эффективное действие для теории струн, скажем в $(D+1)$-мерном пространстве, и редуцировать его в $D$-мерное, то размер компактного измерения появится как множитель перед эффективным ( $D$-мерным) действием, который можно интерпретировать как константу связи. Это дает возможность превратить дуальность $R \leftrightarrow \alpha^{\prime} / R$ в соотношение между эффективными теориями, одна из которых находится в режиме сильной, а другая - слабой связи. Мы не будем вдаваться в подробности этой достаточно сложной процедуры, их можно найти, например, в работах [3, 4]. Результатом является предположение, что некоторая квантовая теория поля на данном многообразии и/или в режиме слабой связи эквивалентна ${ }^{2)}$ другой квантовой теории, вообше говоря, на другом многообразии и/или в режиме сильной связи. Удивительно, что, применяя аргументы такого рода к определенным суперсимметричным калибровочным теориям, иногда удается сделать явные предсказания о точном спектре и виде эффективного действия.

1.2. Струны, M-теория, компактификации и D-браны. Обсудим теперь более детально способ построения суперсимметричных четырехмерных (4D) калибровочных теорий исходя из теории струн. Отправной точкой является некоторая суперсимметричная теория струн без аномалий. Существует несколько примеров таких теорий, определенных первоначально как пертурбативные разложения в терминах поляковского континуального интеграла. Их общим свойством является то, что они существуют в 10-мерном пространстве и обладают по крайней мере $\mathcal{N}=1$ десятимерной пространственно-временной суперсимметрией. Более того, оказывается, что эти разные струнные модели можно считать различными пертурбативными фазами некоторой единой теории, связанными преобразованиями дуальности. Кроме того, вне рамок теории возмущения эта единая теория - так называемая М-теория - ведет себя скорее как $11 \mathrm{D}$ теория в пространстве-времени с одним компактным измерением [4].

Чтобы получить макроскопически 4D суперсимметричную калибровочную теорию, следует найти способ редуцирования в 4D пространство. В теории струн существует стандартный путь, восходящий к идее Калуцы-Клейна: полное пространство-время представляется как прямое произведение $4 \mathrm{D}$ евклидова пространства $\mathbb{R}^{4}$ и некоторого компактного многообразия $K$. "Внутреннее" пространство $K$ определяет "цветовые" свойства теории, число 4D суперзарядов и т.п. Суперсимметрия требует, чтобы компактное многообразие $K$ было $3 \mathbb{C}^{-м е р н ы м ~}\left(3_{\mathbb{C}} \mathrm{D}\right)$ комплексным многообразием в $10 \mathrm{D}$ картине или произведением $3_{\mathbb{C}} \mathrm{D}$ комплексного многообразия на $S^{1}$ в $11 \mathrm{D}$ картине. Более

\footnotetext{
2) Эквивалентна в указанном выше смысле. Обычно эквивалентность подразумевает совпадение (частично) спектра и некоторых корреляционных функций в дуальных теориях.
} 
того, оказывается, что в некоторых случаях нетривиальная часть этого $3_{\mathbb{C}} \mathrm{D}$ комплексного многообразия может быть представлена $1_{\mathbb{C}} \mathrm{D}$ комплексной кривой или $2 \mathrm{D}$ вешественной римановой поверхностью $\Sigma$. Начиная с $11 \mathrm{D}$ М-теории следует выбрать конкретную схему компактификации в $4 \mathrm{D}$ так, чтобы полученная теория имела заданную 4D суперсимметрию, требуемую калибровочную группу (в большинстве реальных случаев $S U(N))$ и набор мультиплетов материи. Согласно [5] сушествует сценарий компактификации, в котором комплексная геометрия может быть сформулирована в терминах римановых поверхностей, именно этот сценарий приводит к эффективным теориям Виттена-Зайберга.

Одной из важных составляющих данной конструкции являются так называемые D-бранные конфигурации в непертурбативной теории струн $[3,6]$. D-браны являются классическими ("тяжелыми") объектами, которые могут быть представлены некоторыми гиперповерхностями в пространстве-времени и основной чертой которых является способность взаимодействовать испусканием и поглощением открытых струн (см. рис. 2), даже в теориях, где запрещены открытые струны в “объемном" (bulk) пространстве.

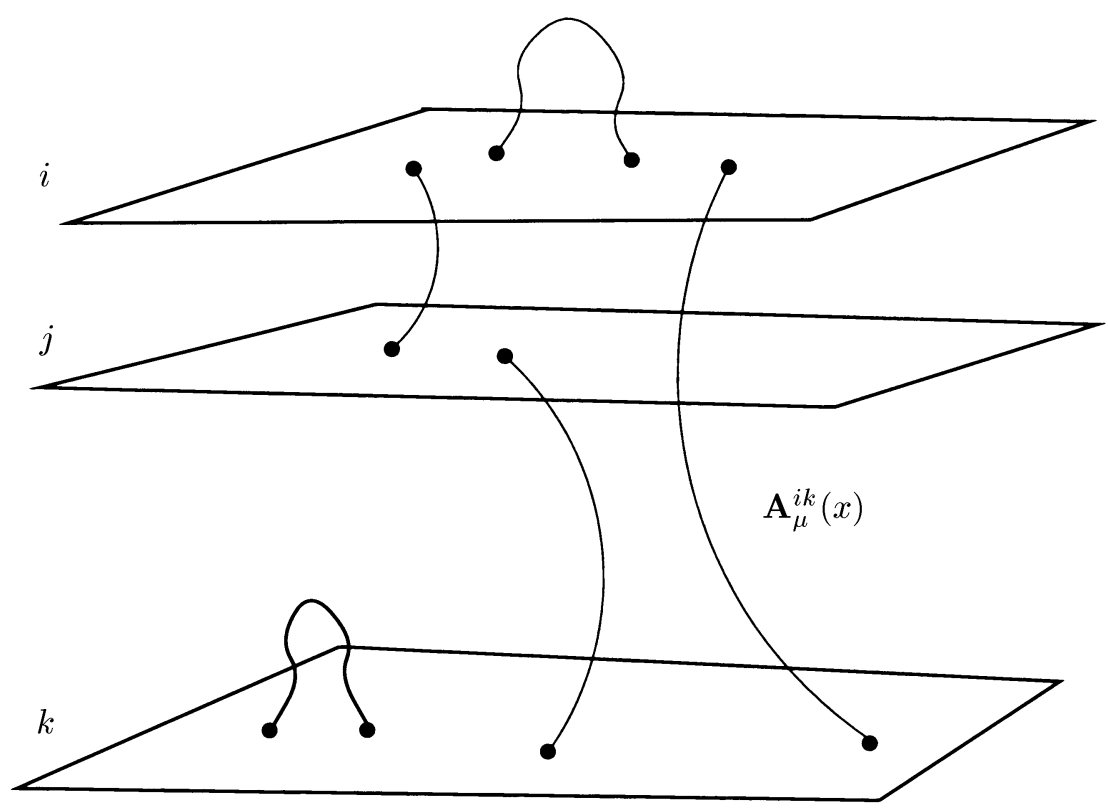

Рис. 2. D-браны. Взаимодействие осуществляется струнами, "приклеенными" концами к различным D-бранам или разным частям одной и той же D-браны. В случае нескольких D-бран естественно возникают именно неабелевы векторные поля в спектре струны, так как векторные поля естественно приобретают дополнительные индексы, нумерующие браны, к которым приклеены соответствующие струны.

Почти очевидно, что конфигурация $N$ D-бран на рис. 2 приводит естественным образом к теории с калибровочной группой $S U(N)(U(N)=S U(N) \otimes U(1)$ с несушественным для полей в присоединенном представлении $U(1)$-фактором), нарушенной до 
$U(1)^{N-1}$. $N$ параллельных D-бран отвечают именно тому, что нужно в этой ситуации, так как струна, натянутая между $i$-й и $j$-й бранами $(i, j=1, \ldots, N)$ (см. рис.2), содержит в точности векторное поле $\mathbf{A}^{i j}$ в спектре. Более того, масса такого векторного поля пропорциональна длине соответствуюшей струны (так как энергия или масса струны пропорциональны ее длине), т.е. расстоянию между $i$-й и $j$-й бранами. Таким образом, безмассовые поля из $U(1)^{N-1}$ отвечают струнам с обоими концами на одной и той же бране, а поля $\mathbf{A}^{i j}$ при $i \neq j$ приобретут хиггсовы массы, пропорциональные вакуумным средним скалярных полей, где эти вакуумные значения будут определяться поперечными координатами D-бран: $\langle\phi\rangle \sim \sqrt{\vec{x}_{\perp}^{2}} / \alpha^{\prime}$.

Следуюший шаг - из $10 \mathrm{D}$ теории струн $\left(\mathbf{A}=\left\|\mathbf{A}^{i j}\right\|-10 \mathrm{D}\right.$ калибровочное поле в теории струн) получить $4 \mathrm{D}$ теорию калибровочных полей. Естественный способ ограничить число пространственных измерений - ограничить теорию на мировой объем $\mathrm{D}$-браны. Мировой объем Дирихле $p$-браны (D $p$-браны) имеет размерность $p+1$, т.е. чтобы получить $4 \mathrm{D}$ калибровочную теорию, следует рассмотреть параллельные D3браны. Этот сценарий вполне возможен и приводит к $\mathcal{N}=4$ суперсимметрии; чтобы получить менее тривиальные теории с $\mathcal{N}=2$ (или даже $\mathcal{N}=1$ ), лучше воспользоваться другой возможностью - так называемой “лестничной” конфигурацией Диаконеску-Ханани-Виттена $[7,8,5]$, когда $N$ параллельных $\mathrm{D} 4$-бран протянуты между двумя вертикальными стенками (рис. 3), так что наивно 5D теория на D4-бране макрокопически (в секторе легких полей) становится четырехмерной, как любая теория Калуцы-Клейна в "ящике" или на окружности.

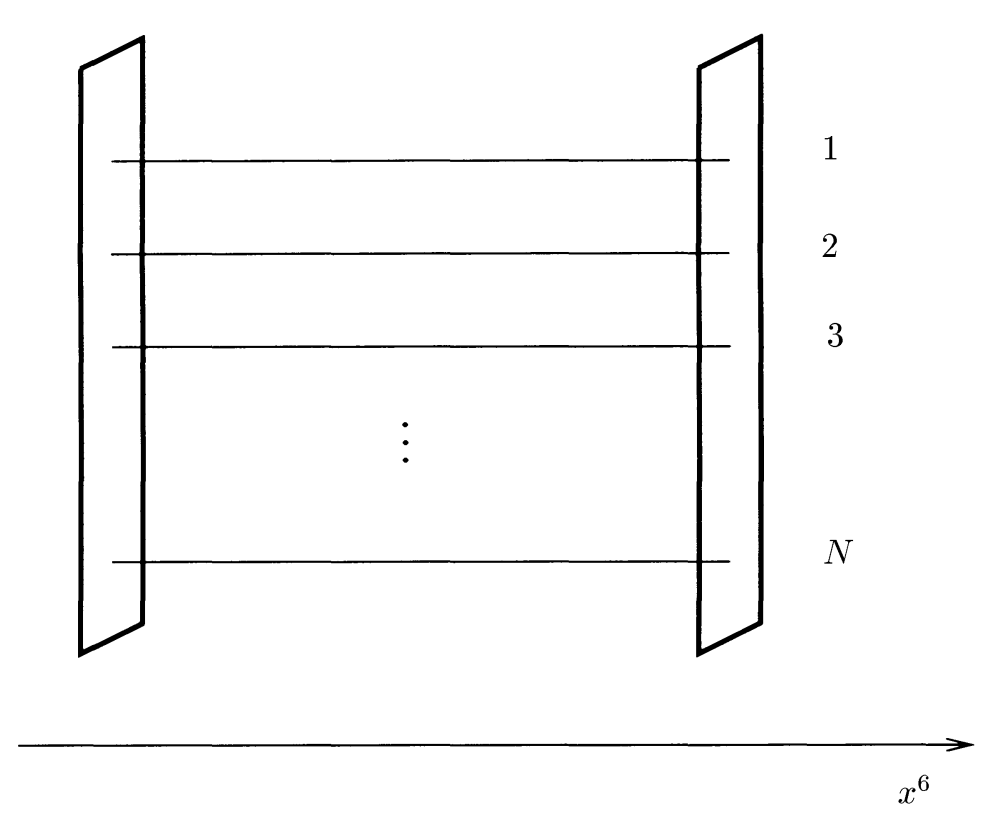

Рис.3. D4-браны, ограниченные 5-бранами до конечного размера (в горизонтальном $x^{6}$-направлении), приводят к макроскопически $4 \mathrm{D}$ теории. 
В роли вертикальных стенок лучше всего взять 5-браны [5], тогда размерные соображения приводят к логарифмическому поведению макроскопической константы связи это обеспечивается в первом приближении тем, что соответствующая компактная координата $\left(x^{6}\right)$ является логарифмической функцией поперечных направлений, поскольку удовлетворяет двумерному уравнению Лапласа, где эффективное двумерие - положение “конщов" D4-бран на 5-бранах.

Представление D4- и 5-бранами в 10D пространстве верно буквально лишь в (квази)классическом приближении. В частности, оно имеет сингулярности в точках, где D4-браны пересекаются с 5-бранами. Эти сингулярности были красиво разрешены в [5], где было предложено “поднять” всю конфигурацию в пространство-время 11D М-теории и считать D4-браны 5-браной М-теории, компактифицированной на окружность 11-го измерения с координатой $x^{10}$. При этом изображенная на рис. 3 конфигурация преврашается в поверхность “шведской стенки” и, если забыть про макроскопические направления $x^{0}, \ldots, x^{3}$, устроена как (некомпактная) риманова поверхность с весьма специальными свойствами (рис. 4). Другими словами, мы получили 5-брану, параметризованную $\left(x^{0}, x^{1}, x^{2}, x^{3}, x^{6}, x^{10}\right)$, которая, если отбросить четыре плоских направления $\left(x^{0}, x^{1}, x^{2}, x^{3}\right)$, устроена как $N$ цилиндров $R \times S^{1}$, расположенных в пространстве-времени вдоль, скажем, координат $\left(x^{6}, x^{10}\right.$ ) (мы будем также использовать соответствующую комплексную координату $\left.z=x^{6}+i x^{10}\right)$. Цилиндры "раздвинуты" в пространстве $V^{\perp}=\left(x^{4}, x^{5}, x^{7}, x^{8}\right)$, но склеены вместе (см. рис. 4), эффективное 2D подпространство $V^{\perp}$ мы будем описывать комплексной координатой $\lambda=x^{4}+i x^{5}$.

1.3. Теория струн и системы Янга-Миллса-Хиггса. Как следует из общей D-бранной картины, пространства модулей суперсимметричных калибровочных теорий могут быть описаны на языке (собственных значений) скалярных полей из супермультиплетов и вильсоновских петель калибровочных полей, когда в пространстве-времени имеются компактные направления. В струнной картине скалярные поля возникают как положения бран (гиперповерхностей) в некоторых дополнительных направлениях, а калибровочные теории на мировых объемах бран являются следствием взаимодействия открытых струн, оканчивающихся на бранах. Квантовые пространства модулей бран описываются полными матрицами (а не только их собственными значениями) [6] с потенциалом ${ }^{3}$

$$
V(\boldsymbol{\Phi})=\operatorname{Tr} \sum_{i<j}\left[\Phi_{i}, \Phi_{j}\right]^{2}
$$

который возникает в результате компактификации $10 \mathrm{D} \mathcal{N}=1$ суперсимметричной теории Янга-Миллса

$$
\operatorname{Tr} \int_{d^{10} x} \mathbf{F}_{M N}^{2}+\text { фермионы }
$$

\footnotetext{
3) Пространство модулей может быть также описано с помощью голоморфного суперпотенциала, например, для шести вешественных полей

$$
W(\boldsymbol{\Phi})=\operatorname{Tr} \epsilon_{i j k} \widetilde{\Phi}_{i}\left[\widetilde{\Phi}_{j}, \widetilde{\Phi}_{k}\right]
$$

где $\widetilde{\Phi}_{j}=\Phi_{j_{1}}+i \Phi_{j_{2}}-$ комплексные скаляры.
} 


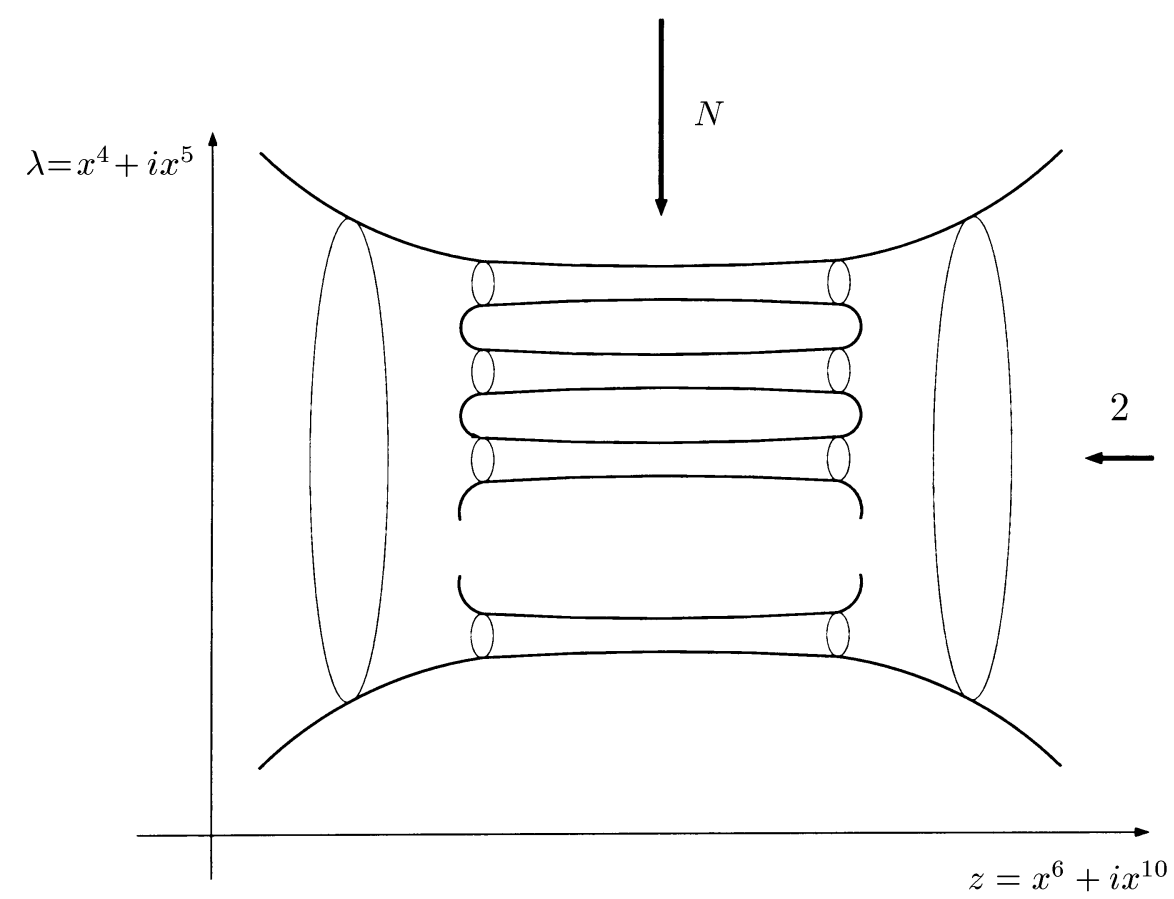

Рис.4. Конфигурация бран, представляющая результат разрешения “лестничной” конфигурации (рис. 3); “лестница" превращается в "шведскую стенку" - гиперэллиптическую риманову поверхность, являюшуюся одновременно $N$-кратным накрытием горизонтального цилиндра.

до 4D $(\mathcal{N}=4$ суперсимметричной $)$ теории

$$
\operatorname{Tr} \int_{d^{4} x} \mathbf{F}_{\mu \nu}^{2}+\left(D_{\mu} \Phi_{i}\right)^{2}+\sum_{i<j}\left[\Phi_{i}, \Phi_{j}\right]^{2}+\text { фермионы, }
$$

где $M=(\mu, i)$, т.е. $A_{\mu}=A_{M}$ для $M=0, \ldots, 3$ и $\Phi_{i}=A_{M}$ при $M=4, \ldots, 9$. В вакуумном секторе фермионными членами в (5) можно пренебречь и, следовательно, говорить о теории Янга-Миллса-Хиггса. Минимумы потенциала (3) отвечают $\left[\Phi_{i}, \Phi_{j}\right]=0$ или одновременно диагонализуемым матрицам

$$
\Phi_{i}=\operatorname{diag}\left(\phi_{1}^{(i)}, \ldots, \phi_{N}^{(i)}\right) .
$$

Разные собственные значения отвечают бранам, разведенным в дополнительных пространственных измерениях. Как обсуждалось выше, в обшей ситуации это отвечает калибровочной теории с группой $U(1)^{N-1}$, когда собственные числа $\Phi_{i}$ совпадают, калибровочная симметрия восстанавливается до $S U(N)$. Например, действие (5) буквально отвечает системе $N$ параллельных D3-бран или (бозонному сектору) $\mathcal{N}=4$ суперсимметричной теории полей Янга-Миллса. Шесть скалярных полей $\Phi_{i}$ (макроскопические измерения обозначены $X_{0}, \ldots, X_{3}$ ) могут иметь ненулевые вакуумные средние, 
т.е. размерность пространства модулей $6(N-1)$. D3-браны можно рассматривать как D5-браны $X_{0}, \ldots, X_{5}$, компактифицированные на двумерный тор, если, скажем, измерения $X_{4}$ и $X_{5}$ считать компактными.

В некоторых случаях на пространствах модулей системы Янга-Миллса-Хиггса можно ввести симплектическую структуру, индуцируемую интегралом $\operatorname{Tr} \int \epsilon_{M N} \delta A_{M} \wedge$ $\delta A_{N}$ по некоторому 2-циклу. Выбрав в качестве такого цикла, например, двумерный тор в (компактифицированных) измерениях $\left(x^{4}, x^{5}\right)$, можно ввести комплексифицированные монодромии

$$
\oint A \equiv \oint\left(A_{4}+i A_{5}\right) \quad \text { и } \quad \oint \bar{A} \equiv \oint\left(A_{4}-i A_{5}\right)
$$

и совершить преобразование $T$-дуальности по отношению к одному из этих комплексных направлений $A \rightarrow \Phi$, где $\Phi=\Phi_{4}+i \Phi_{5}-$ комплексный скаляр. Тогда $\int_{T^{2}} \delta \bar{A} \wedge \delta A$ преврашается в $\int_{T^{2}} \delta \bar{A} \wedge \delta \Phi$. Полученный интеграл можно рассматривать как эффективную двумерную затравочную или классическую структуру Янга-Миллса-Хиггса

$$
\operatorname{Tr} \int \delta \bar{A} \wedge \delta \Phi \sim \sum_{i} \delta q_{i} \wedge \delta p_{i},
$$

где $\bar{A} \sim \oint \operatorname{diag}\left(q_{1}, \ldots, q_{N}\right)$, а $p_{i}-$ диагональные элементы $\Phi$

Диагональные элементы $\bar{A}$ и $\Phi$ можно рассматривать как независимые модули (компактифицированной) $\mathcal{N}=4$ суперсимметричной калибровочной теории. При нарушении суперсимметрии до $\mathcal{N}=2$ к (супер)потенциалу следует добавить дополнительные массовые члены для двух из трех скалярных полей, и размерность "скалярного" пространства модулей уменьшается до $2(N-1)$ или одного комплексного диагонального скаляра (6). Кроме того, в отличие от теории с $\mathcal{N}=4$, в обшем случае (нетривиальных граничных условий) матрищы скалярных полей и монодромий становятся зависимыми друг от друга, или удовлетворяют нетривиальным коммутационным соотношениям вида

$$
[\bar{A}, \Phi] \sim m J
$$

линейным по параметру "массовой деформации" [9] (при $m \rightarrow 0$ восстанавливается $\mathcal{N}=4$ суперсимметрия), где $J$ - некоторая матрица калибровочно-ковариантного вида. Диагонализуя матрищу $\bar{A}$, соотношение (8) можно разрешить относительно $\Phi$.

Квантовые эффекты превращают затравочную симплектическую форму (7) в

$$
\sum_{k} \delta q_{k} \wedge \delta p_{k} \sim \sum_{k} \delta \vartheta_{k} \wedge \delta a_{k}
$$

где

$$
a_{i}=\oint_{A_{i}} d S
$$

- "правильные" квантовые переменные - интегралы Виттена-Зайберга. Замену $a_{i}=$ $a_{i}(\Phi, \Lambda)$ можно рассматривать как переход от затравочных величин (собственных значений $\Phi)$ к их точным квантовым аналогам в эффективных теориях $M_{W}^{(0)} \rightarrow M_{W} \sim$ $\left(a_{i}-a_{j}\right)$. Так же можно понимать и преобразование $q_{i} \rightarrow \vartheta_{i}-$ как преобразование от затравочных значений монодромии к ее точному квантовому виду в эффективных теориях. 
1.4. Эффективная теория Виттена-Зайберга. Теорией Виттена-Зайберга $[10,11]$ обычно называют конструкцию низкоэнергетических (абелевых) $\mathcal{N}=2$ суперсимметричных калибровочных теорий в четырех (или в более обшем случае от трех до шести) измерениях для $\mathcal{N}=2$ суперсимметричных теорий Янга-Миллса с затравочным действием

$$
\mathcal{L}=\operatorname{Tr} \int d^{4} \vartheta \hat{\mathcal{L}}(\mathbf{\Phi})=\frac{1}{g^{2}} \operatorname{Tr} \mathbf{F}_{\mu \nu}^{2}+i \theta \operatorname{Tr} \mathbf{F}_{\mu \nu} \widetilde{\mathbf{F}}_{\mu \nu}+\cdots
$$

Точные непертурбативные утверждения [10-12] содержат информацию о спектре массивных БПС-возбуждений ${ }^{4}$ ("W-бозонов" и монополей) и о вильсоновом эффективном действии безмассовых полей (см., например, $[13,14])$.

Суперсимметрия приводит к сильным ограничениям на вид эффективного действия. В случае $\mathcal{N}=1$ не перенормируется классический суперпотенциал (и это позволяет изучать множество вакуумов теории - критических точек суперпотенциала), в то время как кинетические члены могут быть записаны с помошью кэлеровой метрики или кэлерова потенциала. Для расширенной суперсимметрии ограничений еше больше, а именно, не сушествует абелевых потенциальных членов (это означает, что вместо набора вакуумных точек имеется непрерывное параметрическое семейство модулей вакуумов), а эффективное действие, например для векторных супермультиплетов, описывается с помошью одной голоморфной функции нескольких комплексных переменных препотенциала $[15,10,11]$.

Для $\mathcal{N}=2$ суперсимметричной теории Янга-Миллса результат выглядит следующим образом. Скалярный потенциал (3) в $\mathcal{N}=2$ теории имеет вид $V(\phi)=\operatorname{Tr}\left[\phi, \phi^{\dagger}\right]^{2}$, и его минимумы после факторизации по калибровочной группе отвечают диагональным $\left(\left[\boldsymbol{\phi}, \phi^{\dagger}\right]=0\right)$, в теории с калибровочной группой $S U(N)$ - бесследовым матрицам

$$
\phi=\left.\left(\begin{array}{cccc}
\phi_{1} & & & \\
& \phi_{2} & & \\
& & \ddots & \\
& & & \phi_{N}
\end{array}\right)\right|_{\operatorname{Tr} \phi=\sum \phi_{j}=0}
$$

инварианты которых

$$
\operatorname{det}(\lambda-\phi)=P_{N}(\lambda)=\sum_{k=0}^{N} u_{k} \lambda^{k}=\prod_{i=1}^{N}\left(\lambda-\phi_{i}\right)
$$

(полное число алгебраически независимых инвариантов $-\operatorname{rank} S U(N)=N-1$ ) параметризуют пространство модулей теории. Благодаря эффекту Хиггса внедиагональная часть матрицы калибровочного поля $\mathbf{A}_{\mu}$ становится массивной, так как условие

$$
\left[\phi, \mathbf{A}_{\mu}\right]_{i j}=\left(\phi_{i}-\phi_{j}\right) \mathbf{A}_{\mu}^{i j}
$$

\footnotetext{
4) БПС(Богомольный-Прасад-Соммерфельд)-состояния образуют “малый” супермультиплет с массами, пропорциональными центральным зарядам расширенной $\mathcal{N} \geqslant 2$ алгебры суперсимметрии.
} 
приводит к массовому члену

$$
\left(\phi_{i}-\phi_{j}\right)^{2}\left(\mathbf{A}_{\mu}^{i j}\right)^{2}
$$

в лагранжиане, в то время как диагональная часть, как следует из (15), остается безмассовой, т.е. калибровочная группа $G=S U(N)$ нарушается механизмом Хиггса до $U(1)^{\operatorname{rank} G}=U(1)^{N-1}$. Результатом является эффективная $\mathcal{N}=2$ абелева калибровочная теория с эффективным лагранжианом $\mathcal{L}_{\text {eff }}\left(\Phi_{i}\right)$, который может быть записан в терминах суперполей

$$
\begin{gathered}
\Phi_{i}=\varphi_{i}+\vartheta \sigma_{\mu \nu} \tilde{\vartheta} f_{\mu \nu}^{i}+\ldots, \\
f_{\mu \nu}^{i} \equiv \partial_{\mu} A_{\nu}^{i}-\partial_{\nu} A_{\mu}^{i}, \quad i=1, \ldots, N-1, \quad \sigma_{\mu \nu}=\left[\gamma_{\mu}, \gamma_{\nu}\right],
\end{gathered}
$$

вакуумные значения которых $\left\langle\varphi_{i}\right\rangle=\phi_{i}$ совпадают с диагональными элементами (12). Поэтому функция комплексных переменных $\mathcal{F}(a)=\left.\mathcal{F}(\phi)\right|_{\sum \phi_{i}=0}$, где в качестве переменных $a_{i}$ в теории возмушения можно выбрать, например, $a_{i}=\phi_{i}-\phi_{N}, i=1, \ldots$ $\ldots, N-1$, действительно определяет эффективное вильсоново действие безмассовых полей с помощью подстановки

$$
\mathcal{L}_{\text {eff }} \sim \operatorname{Im} \int d^{4} \vartheta \mathcal{F}\left(\phi_{i} \rightarrow \Phi_{i}\right)=\operatorname{Im} \frac{\partial^{2} \mathcal{F}}{\partial a_{i} \partial a_{j}}(a) f_{\mu \nu}^{i} f_{\mu \nu}^{j}+\ldots
$$

Этот факт можно проверить явными вычислениями квантовых поправок в стандартной $\mathcal{N}=2$ суперсимметричной калибровочной теории.

В $\mathcal{N}=2$ теории возмушений вычисление эффективных зарядов $T_{i j}$ сводится к однопетлевой диаграмме (рис. 5), так как вклады старших петель равны нулю. Интегрирование по импульсам в петле приводит к результату

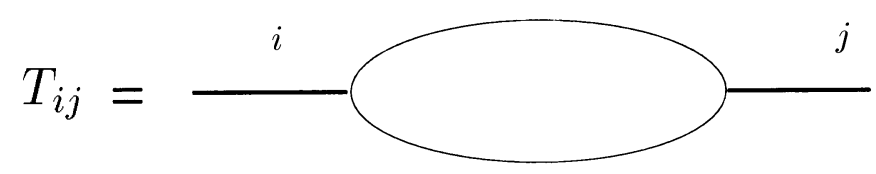

Рис. 5. Однопетлевая диаграмма для вычисления эффективного заряда.

$$
\left(\delta^{2} \mathcal{F}_{\text {pert }}\right)_{i j}=\left(\delta^{2} \mathcal{F}_{1 \text {-loop }}\right)_{i j}=T_{i j} \sim \sum \ln \frac{(\text { mass })^{2}}{\Lambda^{2}}
$$

где $\Lambda \equiv \Lambda_{\mathrm{QCD}}$ - масштабный параметр теории, а сумма в формуле (18) берется по массам распространяющихся в петле полей. Наиболее простым способом результат (18) может быть записан в виде формулы Коулмена-Вайнберга для препотенциала

$$
\mathcal{F}_{1 \text { loop }}=\frac{1}{4} \sum(\text { mass })^{2} \ln \frac{(\text { mass })^{2}}{\Lambda^{2}} .
$$

В чистой теории Янга-Миллса единственным источником масс в (19) является эффект Хиггса (15), поэтому окончательный пертурбативный результат принимает вид

$$
\mathcal{F}_{\text {pert }}=\frac{1}{4} \operatorname{Tr} \phi^{2} \ln \frac{\phi^{2}}{\Lambda^{2}}
$$


То же самое вычисление может быть выполнено и в общем случае: результатом будет сумма членов (20), отвечающих каждому мультиплету; следы в каждом члене должны вычисляться в соответствующем представлении $\operatorname{Tr} \equiv \operatorname{Tr}_{R}$, а знаки - "+" для векторного и “-” для гипермультиплета.

Сложнее обстоит дело с инстантонными вкладами. Известна общая структура эф-фективного действия; соответствующий препотенциал имеет следующее асимптотическое разложение при больших вакуумных средних $a_{\{I\}} / \Lambda \rightarrow \infty$ :

$$
\mathcal{F}=\mathcal{F}_{\text {pert }}+\mathcal{F}_{\text {inst }}=\frac{1}{4} \sum_{\{I\}} a_{\{I\}}^{2} \ln \frac{a_{\{I\}}^{2}}{\Lambda^{2}}+\sum_{\{I\}} a_{\{I\}}^{2} \sum_{k=1}^{\infty} \mathcal{F}_{\{I\}, k}\left(\frac{\Lambda}{a_{\{I\}}}\right)^{2 N k}
$$

с некоторыми коэффициентами $\mathcal{F}_{\{I\}, k}$. Члены с фиксированным $k$ в правой части $(21)$ отвечают сектору с инстантонным числом $k$ в $S U(N)$ теории Янга-Миллса; например, в теории с группой $S U(2)$ интеграл по размерам инстантонов имеет вид $\int d \rho / \rho^{5}$ и для $k$ инстантонов приводит к масштабному фактору $\Lambda^{4 k}$.

Что касается массивных возбуждений $[10,11]$, то, по крайней мере, спектр БПС-состояний

$$
M \sim\left|\mathbf{n a}+\mathbf{m a}_{D}\right|
$$

связан с препотенциалом $\mathcal{F}$ формулами

$$
\mathbf{a}_{D}=\frac{\partial \mathcal{F}}{\partial \mathbf{a}}
$$

Согласно гипотезе Виттена-Зайберга БПС-массы а и $\mathbf{a}_{D}$ выражаются через периоды мероморфного дифференциала $d S$ на вспомогательной римановой поверхности $\Sigma$ и зависят от вакуумных значений скалярных полей, как от определенных координат на пространстве модулей комплексных структур $\Sigma$. В частности, в этих координатах матрица эффективных зарядов $T_{i j}(\mathbf{a})=\partial^{2} \mathcal{F} /\left(\partial a_{i} \partial a_{j}\right)$ играет роль матрииы периодов римановой поверхности $\Sigma$. Например, в случае чистой калибровочной теории с группой $S U(N)$ вспомогательная риманова поверхность и мероморфный дифференциал имеют вид $[10,12]^{5)}$

$$
w+\frac{\Lambda^{2 N}}{w}=2 P_{N}(\lambda), \quad d S=\lambda \frac{d w}{w} .
$$

\footnotetext{
5) Формулы (25) можно пытаться понять (но не вывести!) следующим образом. В теории возмущений массы частиц ( $W$-бозонов и их суперпартнеров) пропорциональны корням $\phi_{i}$ производящего полинома (13). Следовательно, их можно "вычленить" из полинома (13) с помошью формулы вычетов

$$
m_{i j} \sim \oint_{C_{i j}} \lambda d \ln P_{N}(\lambda),
$$

которая для некоторого контура $C_{i j}$ приводит прямо к формуле (15). Контурный интеграл (24) можно рассматривать как интеграл на вырожденной римановой поверхности - $\lambda$-плоскости с $N$ отмеченными точками - корнями полинома (13). Тогда смысл формул (25) заключается в том, что единственным непертурбативным эффектом в терминах римановой поверхности является разрешение сингулярностей наиболее простым возможным способом - заменой отмеченных точек при $\lambda=\phi_{i}$ на "ручки": $w+\Lambda^{2 N} / w \sim \lambda-\phi_{i}$, и совершением таким образом перехода от $\lambda$-плоскости с отмеченными точками к гладкой римановой поверхности.
} 
Ниже мы рассмотрим, как вспомогательные комплексные кривые или римановы поверхности возникают в рамках общего подхода М-теории. Мы также покажем, что препотенциал $\mathcal{F}$ можно отождествить с так назьваемой тау-функцией иерархии интегрируемых уравнений [16], и это означает, что его производные (БПС-массы, эффективные заряды и их производные) удовлетворяют точным непертурбативным нелинейным дифференциальным уравнениям.

\section{2. ИНТЕГРИРУЕМЫЕ СИСТЕМЫ}

Чтобы продолжить обсуждение непертурбативных явлений в теории струн и суперсимметричных калибровочных теориях поля, нам потребуются некоторые определения из теории интегрируемых систем. Мы ограничимся случаем конечномерных интегрируемых систем, когда симплектическая форма может быть приведена к виду конечной суммы

$$
\Omega=\sum_{k} \delta a_{k} \wedge \delta \vartheta_{k}
$$

так что часть переменных $\left\{a_{k}\right\}$ постоянна на траекториях (уравнениях движения), а остальные $\left(\left\{\vartheta_{k}\right\}\right)$ линейны по времени.

Ниже мы будем иметь дело в основном с голоморфными интегрируемыми системами, или системами, в которых нелинейные дифференциальные уравнения могут быть записаны в форме представления Лакса. Они эквивалентны системе операторных соотношений

$$
\frac{\partial \mathcal{L}}{\partial T_{k}}=\left[B_{k}, \mathcal{L}\right]
$$

$(k=1,2, \ldots)$ или условиям их непротиворечивости (уравнениям Захарова-Шабата)

$$
\left[\frac{\partial}{\partial T_{k}}-B_{k}, \frac{\partial}{\partial T_{l}}-B_{l}\right]=0 \quad \forall k, l .
$$

Представление Лакса подразумевает существование вспомогательной линейной задачи вида

$$
\mathcal{L} \Psi=\lambda \Psi
$$

или

$$
\frac{\partial}{\partial T_{k}} \Psi=B_{k} \Psi
$$

и решения уравнений $(27),(28)$ обычно строятся с использованием геометрических свойств $\Psi$-функции - решения линейных уравнений (29), (30).

2.1. Конечнозонные решения: конструкция Кричевера. Важным примером конечномерных интегрируемых систем являются конечнозонные решения интегрируемых моделей типа моделей Кадомцева-Петвиашвили (КП) или цепочек Тоды, обычно фиксируемые дополнительным условием Новикова (см., например, $[17,18]$ )

$$
[\mathcal{L}, \mathcal{A}]=0, \quad \mathcal{A}=\sum_{k} c_{k} B_{k}
$$


где $\mathcal{L}$ и $B_{k}$ - операторы Лакса и эволюции (27), а $c_{k}$ - некоторый конечный набор ненулевых констант. Интегрирование общей конечнозонной задачи задается конструкцией Кричевера [19] и предполагает следующее:

- Совместный спектр коммутирующих операторов $\mathcal{L}$ и $\mathcal{A}(31)$ может быть описан системой алгебраических уравнений, приводящих к комплексной алгебраической кривой $\Sigma$.

- Модули комплексной кривой $\Sigma$ являются интегралами движения системы (31).

- Интегрируюшей заменой переменных является отображение Абеля

$$
\mathbf{A}(P)=\int_{P_{0}}^{P} d \boldsymbol{\omega}
$$

а лиувиллев тор (многообразие угловых переменных) - вешественное сечение якобиана кривой $\Sigma$.

- $\Psi$-функция Бейкера-Ахиезера (БА) является сечением некоторого расслоения над кривой $\Sigma$ (в нашем случае - линейного расслоения) или однозначной функцией с сушественной особенностью и с не менее чем $g$ полюсами на кривой $\Sigma_{g}$ рода $g$. Это означает, что функцию БА можно явно задать следуюшей формулой:

$$
\Psi(P, \mathbf{T})=e^{\sum_{k} T_{k} \Omega_{k}(P)} \frac{\theta\left(\mathbf{A}(P)+\sum_{k} T_{k} \oint_{\mathbf{B}} d \Omega_{k}+\Delta\right) \theta\left(\Delta+\mathbf{A}\left(P_{0}\right)\right)}{\theta(\mathbf{A}(P)+\Delta) \theta\left(\sum_{k} T_{k} \oint_{\mathbf{B}} d \Omega_{k}+\Delta+\mathbf{A}\left(P_{0}\right)\right)}
$$

где

$$
\Omega_{k}(P)=\int_{P_{0}}^{P} d \Omega_{k}
$$

$\left\{d \Omega_{k}\right\}$ - набор канонических мероморфных дифференциалов второго рода (с единственным полюсом $(k+1)$-го порядка), обычно нормированных на нулевые $\mathbf{A}$-периоды:

$$
\oint_{\mathbf{A}} d \Omega_{i}=0, \quad \Delta=\mathbf{K}-\sum_{s=1}^{g} \mathbf{A}\left(\gamma_{s}\right)
$$

где $\mathbf{K}$ - вектор римановых констант, $\left\{\gamma_{s}\right\}$ - дивизор или набор полюсов функции БА, а буквой $\theta$ обозначена тэта-функция Римана. Тау-функция пропорциональна тэта-функции:

$$
\mathcal{T}(\mathbf{T})=e^{\sum_{k l} \gamma_{k l} T_{k} T_{l}} \theta\left(\sum_{k} T_{k} \oint_{\mathbf{B}} d \Omega_{k}+\Delta\right),
$$

- функции на якобиане кривой Jac $\left(\Sigma_{g}\right)$ (подробнее см., например, [20, 21]), а квазипериодические решения интегрируемых уравнений выражаются через производные тэта-функций. Так, например, для решения уравнения КП имеется формула МатвееваИтса

$$
U=\partial^{2} \ln \theta\left(\sum_{k} T_{k} \oint_{\mathbf{B}} d \Omega_{k}+\Delta\right)+\gamma_{11}
$$




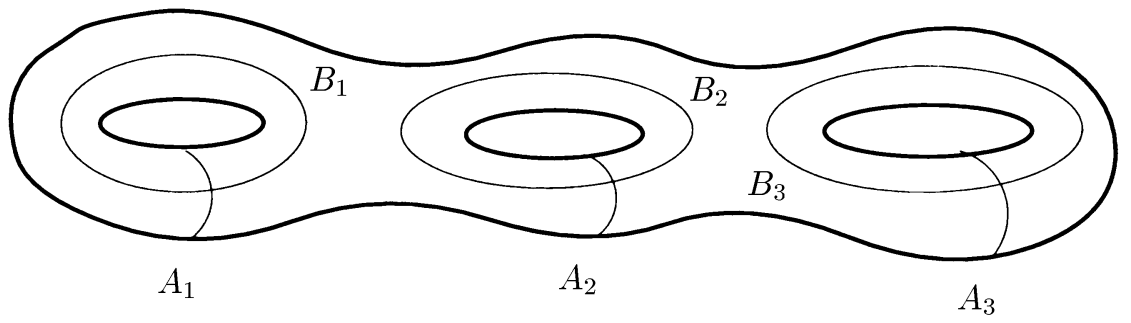

Рис. 6. Комплексная кривая, или двумерная риманова поверхность рода $g=3$. Канонический базис $\mathbf{A}$ - и $\mathbf{B}$-циклов образует форму пересечений $A_{i} \circ B_{j}=\delta_{i j}$.

- Гамильтонова структура на конечнозонных решениях может быть задана с помошью производящего (мероморфного на $\Sigma$ ) 1-дифференциала $d S$, периоды которого (интегралы по нетривиальным циклам на римановой поверхности) задают канонически нормированные интегралы движения динамической системы.

Появляющиеся комплексные кривые можно описать алгебраическими уравнениями, например одним соотношением на две комплексные переменные

$$
\mathcal{P}(\lambda, w)=0,
$$

где $\mathcal{P}$ - определяющий $1_{\mathbb{C}} \mathrm{D}$ комплексное или $2_{\mathbb{R}} \mathrm{D}$ вещественное многообразие полином, коэффициенты которого являются модулями комплексной структуры, или, скажем, системой $n-1$ полиномиальных уравнений на $n$ комплексных переменных. Топологически любая комплексная кривая может быть охарактеризована одним неотрицательным целым числом - родом $g$ (количеством “ручек", см. рис.6), и для фиксированного рода комплексная структура может быть параметризована $3 g-3$ комплексными числами - модулями комплексной структуры, т.е. размерность пространства модулей $\operatorname{dim}_{\mathbb{C}} \mathcal{M}_{g}=3 g-3$.

Исходя из обшего подхода к интегрируемым системам, основанного на "парах Лакса", алгебраические уравнения кривых могут быть получены следуюшим способом. Отправной точкой является условие Новикова (31) взаимной коммутативности двух операторов $\mathcal{L}$ и $\mathcal{A}$. На практике это означает, что эти два оператора функционально зависимы, или сушествует такая функция этих операторов, что $\mathcal{P}(\mathcal{L}, \mathcal{A})=0$. Действительно, при переходе от операторов к собственным значениям в простых случаях эта функция прямо приводит к уравнению спектральной кривой в форме (35). Фактически следует сначала перейти к собственным функциям одного из операторов, скажем $\mathcal{A}$, как к конечномерному базису в линейном пространстве функций БА, при этом действие другого оператора $\mathcal{L}$ в этом базисе может быть записано в виде конечномерной матрицы $\mathcal{L}_{i j}(w)$, явно зависящей от собственного значения $w$ первого оператора. Характеристическое уравнение вспомогательной линейной задачи принимает вид

$$
\operatorname{det}(\lambda-\mathcal{L}(w))=\mathcal{P}(\lambda, w)=0
$$


и дает явно алгебраическое уравнение комплексной кривой $\Sigma$. Входяшая в (36) функция не обязательно является полиномом, но, вводя дополнительные переменные, это уравнение можно переписать в виде системы полиномиальных уравнений.

Уравнение (36) определяет спектральную кривую $\Sigma$ как накрытие над некоторой затравочной кривой $\Sigma_{0}{ }^{6}$, параметризуемой переменной $w$ - собственным значением оператора $\mathcal{A}$, в то время как другой оператор $\mathcal{L}=\mathcal{L}(w)$ становится явно зависимым от этого спектрального параметра. Это означает, что конечнозонная конструкция переводит задачу бесконечномерной интегрируемой системы (решения иерархий типа КП или Тоды) в типичную задачу для конечномерных систем (см., например, [22]) с оператором Лакса, который зависит от спектрального параметра и, следовательно, определен на некоторой затравочной кривой. Очевидно, что представление в виде накрытия, вообше говоря, может быть выбрано не единственным способом - например, можно начать ту же процедуру с собственных значений и собственных функций другого оператора.

Конечнозонные интегрируемые системы обычно отвечают $g$-параметрическим (или $\leqslant g$ ) семействам комплексных кривых (так, чтобы размерность соответствуюшего подпространства в пространстве модулей была равна размерности якобиана, т.е. чтобы количество независимых интегралов движения совпадало с количеством угловых переменных ${ }^{7)}$ ). Размерность якобиана определяется числом независимых (глобально определенных) голоморфных дифференциалов $d \omega_{i}, \quad i=1, \ldots, g$, и совпадает с родом кривой $\Sigma_{g}$. На кривой $\Sigma_{g}$ сушествуют $2 g$ независимых нестягиваемых контуров (по два вокруг каждой "ручки", см. рис. 6), которые можно канонически разбить на так назы-

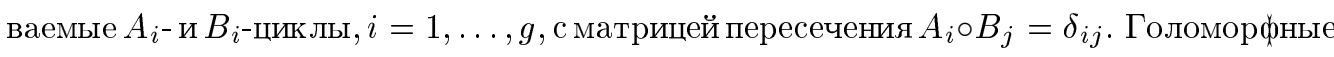
дифференциалы обычно выбираются нормированными на $\mathbf{A}$-циклы:

$$
\oint_{A_{j}} d \omega_{i}=\delta_{i j}
$$

тогда интегралы по $\mathbf{B}$-циклам дают матрицу периодов

$$
\oint_{B_{j}} d \omega_{i}=T_{i j}, \quad \int_{\Sigma_{g}} d \omega_{i} \wedge \overline{d \omega}_{j}=2 i \operatorname{Im} T_{i j}
$$

Матрица периодов (38) симметрична, это легко проверить, применяя теорему Стокса:

$$
0=\int_{\Sigma_{g}} d \omega_{i} \wedge d \omega_{j}=\sum_{k=1}^{g} \oint_{A_{k}} d \omega_{i} \oint_{B_{k}} d \omega_{j}-\sum_{k=1}^{g} \oint_{A_{k}} d \omega_{j} \oint_{B_{k}} d \omega_{i}=T_{i j}-T_{j i}
$$

\footnotetext{
${ }^{6)}$ Конечно, обозначение $\Sigma_{0}$ не подразумевает, что затравочная кривая обязательно должна быть нулевого рода. В то же время мы увидим, что в большинстве известных примеров затравочная кривая $\Sigma_{0}$ является сферой или тором, т.е. кривыми рода $g_{0}=0,1$.

7) На самом деле $g$-параметрические семейства возникают в простейших случаях (в контексте данной работы это отвечает теориям с калибровочной группой $S U(N))$. В общем случае следует рассматривать многообразия Прима, являющиеся фактором якобиана относительно некоторой инволюции.
} 
Конечнозонное решение интегрируемой системы может быть задано производяшим дифференциалом $d S$ (дифференциалом Якоби " $p d q$ ") на спектральной кривой $\Sigma$ [22], производные которого по $g$ независимым направлениям в пространстве модулей являются некоторыми линейно независимыми голоморфными дифференциалами:

$$
\delta_{\text {moduli }} d S \text { есть голоморфньй дифференциал. }
$$

Легко видеть, что голоморфные дифференциалы (40) станут канонически нормированными (37), если в качестве координат на пространстве модулей выбрать канонические переменные интегралы движения - переменные действия или $\mathbf{A - п е р и о д ы ~ д и ф ф е р е н ц и а - ~}$ ла $d S$

$$
a_{j}=\oint_{A_{j}} d S
$$

Действительно,

$$
\delta a_{j}=\oint_{A_{j}} \frac{\partial d S}{\partial a_{i}} \delta a_{i}
$$

и, сравнивая (42) с (37), получаем

$$
\frac{\partial d S}{\partial a_{i}} \cong d \omega_{i}
$$

где знак $\cong$ означает равенство с точностью до полных производных, обрашаюшихся в нуль при интегрировании по замкнутому контуру. "Дуальные” В-периоды принято обозначать $\mathbf{a}^{D}$ :

$$
a_{j}^{D}=\oint_{B_{j}} d S
$$

Дифференцируя обе части равенства по $a_{i}$ и используя (43) и (38), получаем

$$
\frac{\partial a_{j}^{D}}{\partial a_{i}}=\oint_{B_{j}} \frac{\partial d S}{\partial a_{i}}=\oint_{B_{j}} d \omega_{i}=T_{i j} .
$$

2.2. Системы Хитчина. Рассмотренная в п. 2.1 конечнозонная конструкция, к сожалению, не имеет никакой естественной геометрической структуры, лежашей в основе интегрируемости. В частности, она не обладает алгоритмом выбора необходимых $g$-мерных подпространств в $(3 g-3)$-мерном пространстве модулей $\mathcal{M}_{\Sigma}$. Интересная геометрическая интерпретация некоторых интегрируемых систем была предложена в [23]. Конструкция Хитчина существенно конечномерна и с самого начала явно использует сушествование затравочной кривой $\Sigma_{0}$, пространство модулей $\mathcal{M}_{\Sigma}$ при этом содержит естественный $g$-мерньй "кусок" - модули накрытия или голоморфного векторного $G$-расслоения $E$ над базовой кривой $\Sigma_{0}$.

Пусть $\overline{\mathbf{A}} \in \mathcal{G}$ (где $\mathcal{G}$ - алгебра Ли комплексной группы $G$ ) является связностью в расслоении $E$. Рассмотрим пространство пар $(\overline{\mathbf{A}}, \Phi)$, где $\Phi$ голоморфно, т.е. удовлетворяет уравнению Гаусса

$$
\overline{\mathbf{D}} \Phi=\bar{\partial} \Phi+[\overline{\mathbf{A}}, \Phi]=0 .
$$


Легко видеть, что кокасательное расслоение к пространству связностей $\mathbf{A}$ с естественной (калибровочно-инвариантной) симплектической структурой

$$
\Omega=\operatorname{Tr} \int_{\Sigma_{0}} \delta \Phi \wedge \delta \overline{\mathbf{A}}
$$

принадлежит расслоению Хиггса, т.е. определенному выше пространству пар.

Интегрируемые системы Хитчина возникают на кокасательном расслоении $T^{*} M_{A}$, где

$$
M_{A}=\frac{E}{\mathrm{G}}
$$

- пространство модулей голоморфных (полустабильных векторных расслоений ${ }^{8)}$ ), $\mathrm{G}$ - калибровочная группа. На $T^{*} M_{A}$ имеется естественная симплектическая структура, которую можно получить факторизацией (47) по калибровочной группе. Соответствуюшая интегрируемая система возникает как редукция свободного движения на $T^{*} E$, генерируемого гамильтонианами $\operatorname{Tr} \Phi^{k}$ :

$$
\left\{\operatorname{Tr} \Phi^{n}, \operatorname{Tr} \Phi^{m}\right\}_{\Omega}=0,
$$

где скобка Пуассона определена симплектической структурой (47) (как обратная матрица), редуцированной на $T^{*} M_{A}$.

Интегралами движения, или переменными действия являются функции на $M_{A}$, взаимно коммутируюшие относительно пуассоновой структуры (47). Угловыми переменными являются координаты на якобиане (многообразии Прима) $\operatorname{Jac}(\Sigma)$ кривой $\Sigma$, накрываюшей $\Sigma_{0}$ таким образом, что голоморфное векторное расслоение $E$ над $\Sigma_{0}$ может быть "линеаризовано" на $\Sigma$. На практике это означает, что кривая $\Sigma$ определяется уравнением

$$
\operatorname{det}(\lambda-\Phi(z))=0
$$

где $z$-некоторая координата на $\Sigma_{0}$. Обычно $\Phi(z)$ можно построить явно, приведя калибровочным преобразованием матрицу $\overline{\mathbf{A}}$ к диагональному виду и решив уравнение (46). Как было замечено в [24], конкретные примеры конечнозонных систем отвечают системам Хитчина на вырожденных кривых с отмеченными точками. При этом уравнение (46) приобретает ненулевую правую часть:

$$
\overline{\mathbf{D}} \Phi=\bar{\partial} \Phi+[\overline{\mathbf{A}}, \Phi]=\sum_{\alpha} J^{(\alpha)} \delta^{(2)}\left(z-z_{\alpha}\right) .
$$

Матричные элементы диагональной матрицы $\overline{\mathbf{A}}$ обычно играют роль координат, а сопряженными переменными (импульсами) являются диагональные элементы $\Phi$. Например, $S L(N)$-система Хитчина на эллиптической кривой

$$
\begin{gathered}
y^{2}=\left(x-e_{1}\right)\left(x-e_{2}\right)\left(x-e_{3}\right), \\
x=\wp(z), \quad y=\frac{1}{2} \wp^{\prime}(z), \quad d z=\frac{d x}{2 y},
\end{gathered}
$$

\footnotetext{
8) Мы не будем вдаваться в детали, отсылая интересующегося читателя к оригинальной работе [23].
} 
с $p$ отмеченными точками $z_{1}, \ldots, z_{p}$ после диагонализации $\overline{\mathbf{A}}=\operatorname{diag}\left(q_{1}, \ldots, q_{N}\right)$ принимает вид уравнения [25-27]

$$
\bar{\partial} \Phi_{i j}+\left(q_{i}-q_{j}\right) \Phi_{i j}=\sum_{\alpha=1}^{p} J_{i j}^{(\alpha)} \delta\left(z-z_{\alpha}\right), \quad i, j=1, \ldots, N,
$$

решением которого является ${ }^{9}$

$$
\begin{aligned}
\Phi_{i j}(z)= & \delta_{i j}\left(p_{i}+\sum_{\alpha} J_{i i}^{(\alpha)} \partial \ln \theta_{*}\left(z-z_{\alpha} \mid \tau\right)\right)+ \\
& +\left(1-\delta_{i j}\right) e^{q_{i j}(z-\bar{z})} \sum_{\alpha} J_{i j}^{(\alpha)} \frac{\theta_{*}\left(z-z_{\alpha}+\frac{\operatorname{Im} \tau}{\pi} q_{i j}\right) \theta_{*}^{\prime}(0)}{\theta_{*}\left(z-z_{\alpha}\right) \theta_{*}\left(\frac{\operatorname{Im} \tau}{\pi} q_{i j}\right)}, \quad q_{i j} \equiv q_{i}-q_{j} .
\end{aligned}
$$

Экспоненциальный (неголоморфный) фактор может быть убран калибровочным преобразованием

$$
\Phi_{i j}(z) \rightarrow\left(U^{-1} \Phi U\right)_{i j}(z)
$$

с матрицей $U_{i j}=e^{q_{i j} \bar{z}}$. Матрица $J_{i j}^{(\alpha)}$ удовлетворяет дополнительным условиям

$$
\sum_{\alpha=1}^{p} J_{i i}^{(\alpha)}=0
$$

имеющим ясный смысл - обращение в нуль суммы всех вычетов $\Phi_{i i}$, и

$$
\operatorname{Tr} J^{(\alpha)}=m_{\alpha},
$$

где $m_{\alpha}=$ const - некоторые параметры (“массы”) в теории. Уравнение спектральной кривой принимает вид

$$
\mathcal{P}(\lambda ; z) \equiv \operatorname{det}_{N \times N}(\lambda-\Phi(z))=\lambda^{N}+\sum_{k=1}^{N} \lambda^{N-k} f_{k}(z)=0,
$$

где $f_{k}(z) \equiv f_{k}(x, y)$ - некоторые функции (в обшем случае с $k$ полюсами) на эллиптической кривой (52). Если, однако, $J^{(\alpha)}$ выбрать так, что

$$
\operatorname{rank} J^{(\alpha)} \leqslant l, \quad l<N,
$$

то функции $f_{k}(z)$ будут иметь полюсы в точках $z_{1}, \ldots, z_{p}$ степени не выше $l$. Вычеты производящего дифференциала

$$
d S=\lambda d z
$$

в отмеченных точках $\left(z_{\alpha}, \lambda^{(i)}\left(z_{\alpha}\right)\right)$ (различные $i$ отвечают выбору различных листов накрывающей поверхности) связаны с массовыми параметрами (57)

$$
m_{\alpha}=\left.\left.\operatorname{Res}\right|_{\mathrm{z}_{\alpha}} \lambda d z \equiv \sum_{i=1}^{N} \operatorname{Res}\right|_{\pi_{(\mathrm{i})}^{-1} \mathrm{z}_{\alpha}} \lambda^{(i)}(z) d z=\left.\operatorname{Res}\right|_{\mathrm{z}_{\alpha}} \operatorname{Tr} \Phi d z .
$$

Легко видеть, что обший вид кривой (58) совпадает с видом кривых, предложенных в [5], по крайней мере, для $l=1$, т.е. для источников ранга 1 (для рационального случая тор вырождается в цилиндр). Система Хитчина с $p=1, l=1$ эквивалентна эллиптической модели Калоджеро-Мозера.

\footnotetext{
${ }^{9)}$ Как обычно, $\theta_{*}(z \mid \tau) \equiv \theta_{11}(z \mid \tau)$ обозначает единственную на торе нечетную $\left(\theta_{*}(0 \mid \tau)=0\right)$ тэта-функцию.
} 
2.3. Деформации конечнозонных решений и тау-функция иерархии Уизема. Перейдем теперь к вопросу, как конечнозонные решения интегрируемых уравнений типа КП или Тоды зависят от модулей соответствующих комплексных кривых. Так как модули являются интегралами движения конечнозонных систем или систем хитчиновского типа, для ответа на этот вопрос следует изучать деформации конечнозонных решений. Другими словами, следует перейти от изучения изоспектральны деформаций, сохраняюших вид вспомогательной линейной задачи или уравнения Лакса (29), к изомонодромным деформациям, которые меняют форму кривой $\Sigma$, но сохраняют периоды некоторых дифференциальных форм.

Подобные деформации изучались исторически впервые в контексте "модулящии" решений задачи Коши для уравнения Кортевега-де Фриза (КдФ) [28] (см. также [17]). Предложенный метод (метод усреднения Боголюбова-Уизема, см., например, [28-32]) напоминает процедуру вычисления эффективного действия в квантовой теории поля: переменные делятся на “быстрые" (тяжелые) и “медленные" (легкие), после чего производится усреднение (интегрирование) по быстрым переменным. Результатом является некоторая эффективная динамика медленных переменных, или эффективное действие для легких мод. Прямое применение метода усреднения приводит к серьезным техническим трудностям, вместо этого можно воспользоваться методом [33], позволяющим сформулировать результат усреднения чисто геометрическим способом, в терминах изомонодромной задачи для некоторых дифференциальных форм на комплексных многообразиях.

Согласно [33] начальными данными являются некоторая локальная система функций $\Omega_{A}$ на комплексной кривой с локальной координатой $\xi$ в окрестности некоторой точки $P$ и соответствуюший им набор параметров $t_{A}$, так что можно ввести 1 -форму

$$
\omega=\sum_{I} \Omega_{I}(P, \mathbf{t}) \delta t_{I},
$$

зависящую от точки $P \in \Sigma$ и параметров $\mathbf{t}$. Уравнениями Уизема называются уравнения

$$
\delta \boldsymbol{\omega} \wedge \delta \boldsymbol{\omega}=0, \quad \delta \boldsymbol{\omega}=\partial_{\xi} \Omega_{I} \delta \xi \wedge \delta t_{I}+\partial_{J} \Omega_{I} \delta t_{I} \wedge \delta t_{J}
$$

где следует независимо проверять равенство нулю членов при $\delta t^{4}$ и $\delta t^{3} \delta \xi$. Обрашение в нуль второго члена приводит к равенству

$$
\sum \partial_{\xi} \Omega_{I} \partial_{J} \Omega_{C}=0
$$

для антисимметризованной суммы, или, вводя переменные Дарбу

$$
t_{I_{0}} \equiv x, \quad \Omega_{I_{0}}(P, \mathbf{t}) \equiv p
$$

зависяшие от конкретного выбора параметров $\left\{t_{I}\right\}$, получим

$$
\begin{gathered}
\partial_{I} \Omega_{J}-\partial_{J} \Omega_{I}+\left\{\Omega_{I}, \Omega_{J}\right\}=0 \\
\left\{\Omega_{I}, \Omega_{J}\right\} \equiv \frac{\partial \Omega_{I}}{\partial x} \frac{\partial \Omega_{J}}{\partial p}-\frac{\partial \Omega_{J}}{\partial x} \frac{\partial \Omega_{I}}{\partial p} .
\end{gathered}
$$


Явный вид уравнений (66) зависит от выбора локальной координаты $p$. Уравнения $(63),(64)$ и (66) определены лишь локально и имеют огромное количество решений.

Заметим, что уравнения Уизема (66) можно рассматривать как условие совместности системы уравнений

$$
\frac{\partial \lambda}{\partial t_{I}}=\left\{\lambda, \Omega_{I}\right\}
$$

Функция $\lambda$ может быть использована в качестве новой координаты. Если перейти к этой координате, то система (66) принимает вид

$$
\left.\frac{\partial \Omega_{I}(\lambda, t)}{\partial t_{J}}\right|_{\lambda=\mathrm{const}}=\left.\frac{\partial \Omega_{J}(\lambda, t)}{\partial t_{I}}\right|_{\lambda=\mathrm{const}}
$$

или для дифференциалов $d \Omega_{I} \equiv d_{\Sigma} \Omega_{I}=\left(\partial \Omega_{I} / \partial \lambda\right) d \lambda-$

$$
\left.\frac{\partial d \Omega_{I}(\lambda, t)}{\partial t_{J}}\right|_{\lambda=\mathrm{const}}=\left.\frac{\partial d \Omega_{J}(\lambda, t)}{\partial t_{I}}\right|_{\lambda=\mathrm{const}} .
$$

Важным примером $\lambda$-координат являются гиперэллиптические координаты на гиперэллиптических кривых $y^{2}=R(\lambda)=\prod_{\beta}\left(\lambda-r_{\beta}\right)$. Разлагая уравнение (69) около точки $\lambda=r_{\alpha}$, получаем

$$
\frac{\partial r_{\alpha}}{\partial t_{I}}=\left.\frac{d \Omega_{J}}{d \Omega_{I}}\right|_{\lambda=r_{\alpha}} \frac{\partial r_{\alpha}}{\partial t_{J}} \equiv v_{I J}^{(\alpha)}(\mathbf{r}) \frac{\partial r_{\alpha}}{\partial t_{J}} .
$$

Уравнения Уизема в форме (68) предполагают существование потенциала $S$, удовлетворяющего условиям

$$
\left.\Omega_{I}(\lambda, t)\right|_{\lambda=\mathrm{const}}=\left.\frac{\partial S(\lambda, t)}{\partial t_{I}}\right|_{\lambda=\mathrm{const}}
$$

или

$$
\left.d \Omega_{I}(\lambda, t)\right|_{\lambda=\mathrm{const}}=\left.\frac{\partial d S(\lambda, t)}{\partial t_{I}}\right|_{\lambda=\mathrm{const}} .
$$

С помошью этого потенциала 1-форма $\omega$ (62) может быть переписана в виде

$$
\delta \omega=\delta S-\frac{\partial S(\lambda, t)}{\partial \lambda} d \lambda
$$

Интересные примеры решений иерархии уравнений Уизема возникают, когда в качестве базиса функций $\Omega_{I}$ выбираются квазиэнергии конечнозонных решений интегрируемых систем типа КП или Тоды (или, на современном языке, систем хитчиновского типа). Это система (многозначных на кривой) функций, периоды которых определяют фазу конечнозонного решения в формуле (32). Производящий дифференциал (62) при этом отождествляется с

$$
\begin{gathered}
\delta \omega=\delta S-\frac{\partial S(\lambda, t)}{\partial \lambda} d \lambda, \\
S=\int^{P} d S \\
\frac{\partial S}{\partial t_{I}}=\int^{P} \frac{\partial d S}{\partial t_{I}}=\int^{P} d \Omega_{I}=\Omega_{I},
\end{gathered}
$$


где $d S$ - обобщенная " $p d q$ " -форма конечнозонного решения. Обычно в иерархиях КП или Тоды эти функции выбираются "полу"многозначными, т.е. их А-периоды равны нулю: $\oint_{\mathbf{A}} d \Omega_{I}=0$. Уравнения $(74)$, очевидно, выполняются тривиальным образом в случае, когда $\left\{t_{I}\right\}$ - внешние параметры (времена иерархии КП или Тоды), а $\left\{\Omega_{I}\right\}$ не зависят от $\left\{t_{I}\right\}$. Идея заключается в том, что это тривиальное решение $(63),(64)$ и (66) может быть переведено в нетривиальное некоторой деформацией модулей. Практически это означает, что формулы (74) должны оставаться справедливыми и в случае, когда $\left\{\Omega_{I}\right\}$ зависят от $\left\{t_{I}\right\}$ некоторым специальным образом, определяемым уравнениями иерархии Уизема. Другими словами, уравнения Уизема отвечают динамике на пространстве модулей комплексных кривых.

Для иерархии Уизема можно определить аналог тау-функции, производящей функции для решений, которую символически можно определить как [33]

$$
\ln \mathcal{T}_{W} \sim \int_{\Sigma} \bar{d} S \wedge d S
$$

где двумерньй интеграл по поверхности $\Sigma$ в действительности “локализуется"на "неаналитичностях" $S$. В случае однозначного производяшего дифференциала $d S$ формула (75) может быть переписана в виде [33-35]

$$
\ln \mathcal{T}_{W}=\frac{1}{2} \sum_{I} \oint_{A_{I}} d S \oint_{B_{I}} d S .
$$

Определение (75), (76) означает, что введенная тау-функция может быть задана системой уравнений первого порядка по переменным $t_{I}=\oint_{A_{I}} d S$ :

$$
\frac{\partial \ln \mathcal{T}_{W}}{\partial t_{I}}=\oint_{B_{I}} d S .
$$

Старшие производные этих соотношений приводят к формулам

$$
\begin{gathered}
\frac{\partial^{2} \ln \mathcal{T}_{W}}{\partial t_{I} \partial t_{J}}=T_{I J}(t) \\
\frac{\partial^{3} \ln \mathcal{T}_{W}}{\partial t_{I} \partial t_{J} \partial t_{C}}=\left.\operatorname{Res}\right|_{\mathrm{dz}=0} \frac{d \Omega_{I} d \Omega_{J} d \Omega_{C}}{d \lambda d z}
\end{gathered}
$$

где $T_{I J}$ - обобщенная матрица периодов, а соотношение (79) - так называемая формула вычетов.

Препотенциал (или уиземовская тау-функция) может быть определен для любого конечнозонного решения иерархии КП или Тоды, т.е. для следующего набора данных:

1) комплексная кривая (риманова поверхность) рода $g$;

2) набор отмеченных точек $P_{i}$;

3) координаты $\xi_{i}$ в окрестностях отмеченных точек $P_{i}{ }^{10}$;

\footnotetext{
10) Мы рассмотрим простейший случай одной отмеченной точки, для которой $\xi=\xi_{0}=0$.
} 
4) пары дифференциалов (например, $d \lambda, d z)$ с фиксированными периодами.

С этими данными можно определить мероморфные дифференциалы с полюсами в некоторой точке $P_{0}$, имеюшие в локальной координате $\xi: \xi\left(P_{0}\right)=\xi_{0}=0$ вид

$$
d \Omega_{n} \underset{P \rightarrow P_{0}}{=}\left(\xi^{-n-1}+O(1)\right) d \xi, \quad n \geqslant 1 .
$$

Это условие определяет $d \Omega_{n}$ с точностью до некоторой линейной комбинации голоморфных $g$-дифференциалов $d \omega_{i}, i=1, \ldots, g$, и эту неоднозначность можно устранить двумя способами. Первый способ - потребовать, чтобы $\mathbf{A}$-периоды $d \Omega_{n}$ равнялись нулю:

$$
\oint_{A_{i}} d \Omega_{n}=0 \quad \forall i, n
$$

Производящий бидифференциал таких дифференциалов $\left(\zeta \equiv \xi\left(P^{\prime}\right), \xi \equiv \xi(P)\right)$

$$
W(\xi, \zeta)=\sum_{n=1}^{\infty} n \zeta^{n-1} d \zeta d \Omega_{n}(\xi)+\cdots
$$

выражается через главную форму $E\left(P, P^{\prime}\right)[20,21]$ :

$$
W\left(P, P^{\prime}\right)=\partial_{P} \partial_{P^{\prime}} \ln E\left(P, P^{\prime}\right) .
$$

Бидифференциал $W\left(P, P^{\prime}\right)$ имеет полюс второго порядка на диагонали $P \rightarrow P^{\prime}$,

$$
W(\xi, \zeta) \sim \frac{d \xi d \zeta}{(\xi-\zeta)^{2}}+O(1)=\sum_{n=1}^{\infty} n \frac{d \xi}{\xi^{n+1}} \zeta^{n-1} d \zeta+O(1)
$$

Дифференциалы (80) являются производными производяшего дифференциала

$$
d \Omega_{n}=\frac{\partial d S}{\partial t_{n}}
$$

Второй способ - строить $d S$ явно, т.е. наложить условие, подобное условию $(40)$ :

$$
\delta_{\text {moduli }} d \widehat{\Omega}_{n} \text { есть голоморфный дифференциал. }
$$

Мы остановимся на случае, когда число модулей равно роду поверхности. Следуя $[35,36]$, введем производящий дифференциал для дифференциалов $d \widehat{\Omega}_{n}$ с помощью бесконечного числа дополнительных параметров $t_{n}$ :

$$
d S=\sum_{n \geqslant 1} t_{n} d \widehat{\Omega}_{n}=\sum_{i=1}^{g} \alpha_{i} d \omega_{i}+\sum_{n \geqslant 1} t_{n} d \Omega_{n}
$$

Периоды

$$
\alpha_{i}=\oint_{A_{i}} d S
$$


можно рассматривать как определенные координаты на пространстве модулей. Заметим, что эти периоды не совпадают буквально с (41), (44), так как формула (88) определяет $\alpha_{i}$ как функции $u_{k}$ и $t_{n}$, или, наоборот, $u_{k}$ как функции $\alpha_{i}$ и $t_{n}$, так что производные $\partial u_{k} / \partial t_{n}$ нетривиальны. Мы будем считать независимыми координатами $\alpha_{i}$ и $t_{n}$,

$$
t_{n}=\left.\operatorname{Res}\right|_{\xi=0} \xi^{n} d S(\xi)
$$

так что производные по $\alpha_{i}$ вычисляются при постоянных $t_{n}$ и наоборот. В частности,

$$
\frac{\partial d S}{\partial \alpha_{i}}=d \omega_{i}, \quad \frac{\partial d S}{\partial t_{n}}=d \Omega_{n}
$$

Теперь можно ввести уиземовскую тау-функцию (75) или препотенциал $\mathcal{F}\left(\alpha_{i}, t_{n}\right) \equiv$ $\ln \mathcal{T}$ с помощью условий (77):

$$
\frac{\partial \mathcal{F}}{\partial \alpha_{i}}=\oint_{B_{i}} d S, \quad \frac{\partial \mathcal{F}}{\partial t_{n}}=\left.\frac{1}{2 \pi i n} \operatorname{Res}\right|_{0} \xi^{-n} d S
$$

Самосогласованность этих условий следует из (90) и билинейных тождеств Римана. В частности,

$$
\frac{\partial^{2} \mathcal{F}}{\partial t_{m} \partial t_{n}}=\left.\frac{1}{2 \pi i n} \operatorname{Res}\right|_{0} \xi^{-n} \frac{\partial d S}{\partial t_{m}}=\left.\frac{1}{2 \pi i n} \operatorname{Res}\right|_{0} \xi^{-n} d \Omega_{m}=\left.\frac{1}{2 \pi i m} \operatorname{Res}\right|_{0} \xi^{-m} d \Omega_{n} .
$$

Из проведенного вычисления ясно, что определение (91) предполагает, что координаты $\xi$ не меняются при вариации модулей. Это означает, что они отвечают не зависящей от модулей параметризации целого семейства кривых как, например, координаты $\lambda$ или $w$ в случае кривых (25). Так как независимость от модулей координаты $\xi$ должна быть согласована с (86), выбор $\xi$ ограничен и сводится к координате для кривых $(25) w^{ \pm 1 / N}$.

Последнее равенство в (92) (симметричность) является примером соотношений Римана и может быть доказано стандартным способом:

$$
\begin{aligned}
0=\int d \Omega_{m} & \wedge d \Omega_{n}=\oint_{A_{i}} d \Omega_{m} \oint_{B_{i}} d \Omega_{n}-\oint_{A_{i}} d \Omega_{n} \oint_{B_{i}} d \Omega_{m}+ \\
& +\frac{1}{2 \pi i} \operatorname{Res}\left(d \Omega_{m} d^{-1} d \Omega_{n}\right)=\left.\frac{1}{2 \pi i n} \operatorname{Res}\right|_{0} \xi^{-n} d \Omega_{m}-\left.\frac{1}{2 \pi i m} \operatorname{Res}\right|_{0} \xi^{-m} d \Omega_{n}
\end{aligned}
$$

где использованы формулы $(81)$ и $(80)^{11)}$.

Препотенциал является однородной функцией аргументов $a_{i}$ и $t_{n}$ степени 2 ,

$$
2 \mathcal{F}=\alpha_{i} \frac{\partial \mathcal{F}}{\partial \alpha_{i}}+t_{n} \frac{\partial \mathcal{F}}{\partial t_{n}}=\alpha_{i} \alpha_{j} \frac{\partial^{2} \mathcal{F}}{\partial \alpha_{i} \partial \alpha_{j}}+2 \alpha_{i} t_{n} \frac{\partial^{2} \mathcal{F}}{\partial \alpha_{i} \partial t_{n}}+t_{m} t_{n} \frac{\partial^{2} \mathcal{F}}{\partial t_{m} \partial t_{n}}
$$

${ }^{11)}$ Следуя [36], мы используем нормировку

$$
d \Omega_{n} \sim w^{n / N} \frac{d w}{w}=\frac{N}{n} d w^{n / N},
$$

так что вычеты в формулах $(93),(91)$ и (92) умножены на $N / n$, а не $1 / n$. 
Это условие может быть также доказано исходя из (91), (88) и (89) с помощью тождеств Римана. Безусловно, $\mathcal{F}$ не является просто квадратичной функцией $a_{i}$ и $t_{n}$, нетривиальная зависимость от этих переменных возникает через зависимость $d \omega_{i}$ и $d \Omega_{n}$ от модулей $u_{k}$, которые можно считать функциями $\alpha_{i}$ и $t_{n}$. Эти функции можно получить, например, подстановкой (87) в (90):

$$
d \widehat{\Omega}_{n}+t_{m} \frac{\partial d \widehat{\Omega}_{n}}{\partial u_{l}} \frac{\partial u_{l}}{\partial t_{m}}=d \Omega_{n}
$$

T.e.

$$
\left(\sum_{m, l} t_{m} \frac{\partial u_{l}}{\partial t_{m}}\right) \oint_{A_{i}} \frac{\partial d \widehat{\Omega}_{n}}{\partial u_{l}}=-\oint_{A_{i}} d \widehat{\Omega}_{n}
$$

или

$$
\left(\sum_{m, l} t_{m} \frac{\partial u_{l}}{\partial t_{m}}\right) \oint_{A_{i}} \frac{\partial d S}{\partial u_{l}}=-\oint_{A_{i}} d S
$$

Если

$$
\oint_{A_{i}} \frac{\partial d S}{\partial u_{l}}=\oint_{A_{i}} d V_{l} \equiv \Sigma_{i l}
$$

то соотношения

$$
t_{m} \frac{\partial u_{k}}{\partial t_{m}}=\Sigma_{k i}^{-1} \alpha_{i}
$$

могут рассматриваться как решения уравнений Уизема (69), отвечающие анзацу (87) для производяшего дифференциала.

Для конкретных семейств кривых формулы (95)-(99) могут быть приведены к еще более простой форме. Это будет использовано в п. 4.3 при обсуждении аналогов уравнений ренормгруппы в контексте иерархий Уизема.

\section{3. СТРУНЫ И ТЕОРИЯ ВИТТЕНА-ЗАЙБЕРГА}

Обратимся теперь к аналитическому построению кривых Виттена-Зайберга $\Sigma$, исходя из общих постулатов М-теории. Сначала мы обсудим, как геометрия комплексных кривых возникает в рамках D-бранной конструкции [5], а потом, следуя [37, 27], продемонстрируем, как в данном контексте возникают интегрируемые системы. В конце данного раздела мы обсудим проблемы нарушения суперсимметрии и компактификации и покажем, что это проясняет причину появления интегрируемых систем в теории Виттена-Зайберга.

3.1. Комплексные кривые как часть геометрии пространства-времени. Как уже обсуждалось выше (см. рис. 3,4 и п. 1.2), согласно конструкции, использованной в работе [5], следует рассмотреть 5-брану М-теории нетривиальной топологии. Калибровочная группа $S U(N)$ определяется топологией браны, а в низкоэнергетическом режиме она нарушается до абелевой $U(1)^{N-1}$, при этом вакуумные значения скаляров Хиггса (в присоединенном представлении) отождествляются с некоторыми модулями комплексной структуры кривой $\Sigma$ - топологически нетривиальной части 5-браны. 
Выбор римановой поверхности $\Sigma$ достаточно сильно ограничен, если мы хотим получить теорию с нужной (нарушенной) калибровочной симметрией - при этом возникают гиперэллиптические кривые, являюшиеся в то же время $N$-кратными накрытиями цилиндра (см. рис. 4). Появление кривых одновременно в форме 2-листных и $N$-листных накрытий отвечает двум возможным формулировкам анзаца Виттена-Зайберга: на языке $S U(2)$ или $S U(N)$ групп известным как в рамках бранных конструкций $[8,5]$, так и в подходе, основанном на интегрируемых системах (см., например, работу [38] и приведенную в ней библиографию).

Вводя на цилиндре координату $w=e^{z}$, мы видим, что система непересекаюшихся бран (рис.2) описывается не зависимым от z уравнением

$$
P_{N}(\lambda)=\prod_{i=1}^{N}\left(\lambda-\phi_{i}\right)=0
$$

а их “связанное” состояние (рис. 4) - комплексной кривой $\Sigma$ :

$$
\frac{\Lambda^{N}}{2}\left(w+\frac{1}{w}\right)=\Lambda^{N} \operatorname{ch} z=P_{N}(\lambda) .
$$

В пределе слабой связи $\Lambda \rightarrow 0$ (т.е. $1 / g^{2} \sim \ln \Lambda \rightarrow \infty$ ) мы возврашаемся к системе бран $(100)^{12)}$.

Таким образом, мы получили аналитическую формулировку рис. 4 - 5-браны топологии $R^{3} \times \Sigma$, голоморфно вложенной в подпространство $R^{5} \times S^{1}$ (с координатами $\left.x^{1}, \ldots, x^{6}, x^{10}\right)$ полного пространства-времени. Периодической координатой является координата

$$
x^{10}=\arg P_{N}(\lambda)=\operatorname{Im} \ln P_{N}(\lambda)=\sum_{i=1}^{N} \arg \left(\lambda-\lambda_{i}\right) .
$$

3.2. D-браны и интегрируемые системы. Рассмотрим конструкцию Диаконеску-Ханани-Виттена в теории типа IIB [7, 8, 5], когда эффективной теорией на мировом объеме 5-браны является 6D суперсимметричная теория Янга-Миллса. Взаимное расположение цилиндров на рис. 4 описывается координатами в ортогональном пространстве $V^{\perp}\left(x^{4}, x^{5}, x^{7}, x^{8}\right)$, т.е. четверкой скалярных полей $\Phi^{(4)}, \Phi^{(5)}, \Phi^{(7)}, \Phi^{(8)}$ из $6 \mathrm{D}$ векторного супермультиплета. Как обычно, эти поля преобразуются по присоединенному представлению калибровочной группы $S U(N)$, где $N$ - число цилиндров, т.е. координаты $x^{4}, \ldots, x^{8}$ следует заменить на, вообще говоря, некоммутируюшие матришы $\Phi^{(4)}, \ldots, \Phi^{(8)}[6]$. Поля из калибровочного мультиплета отвечают открытым струнам, натянутым между цилиндрами, а соответствующие 10D векторные поля $A_{M}=\left\{A_{s}, A_{i}\right\}$ разлагаются на компоненты с $s=0,1,2,3,6,10$, отвечающие с позиций эффективной теории на бране $6 \mathrm{D}$ векторному полю, и с $i=4,5,7,8$ - четверке упомянутых скаляров.

\footnotetext{
12) Другими словами, для $|z| \ll|\ln \Lambda| \quad \lambda$ практически не зависит от $z$ и локализована около одного из $\phi_{i}$. Только при $|z| \sim|\ln \Lambda|$ координата $\lambda$ может быть "разморожена" и интерполирована между различными $\phi_{i}$.
} 
Неабелево взаимодействие возникает как результат процессов взаимодействия между $i$-й, $j$-й и $k$-й бранами, подобных изображенному на рис. 2 .

В вакуумном состоянии скалярные поля удовлетворяют БПС-условию. Это условие принимает вид

$$
D_{M} \Phi \equiv \partial_{M} \Phi+\left[A_{M}, \Phi\right]=0, \quad F_{M N}=0,
$$

когда только одно из полей $\Phi^{(4)}, \ldots, \Phi^{(8)}$ не равно нулю, в противном случае это уравнение будет также содержать вклады от взаимодействия скаляров, следуюшего из (3) или (2). Это соответствует случаю, изображенному на рис. 4 , где подразумевается, что некоторое скалярное поле, скажем $\Phi \equiv \Phi^{(4)}+i \Phi^{(5)}$, имеет ненулевое, зависящее от $z$, вакуумное среднее, в точности отвечаюшее тому, что цилиндры "разведены" и "склеены". Чтобы объяснить рис. 4, необходимо продемонстрировать, что уравнение (103) имеет нетривиальное решение $\Phi(z) \neq$ const, причиной появления которого являются нетривиальные граничные условия, наложенные на $\Phi$ при $z \rightarrow \pm \infty$.

Чтобы получить адекватное описание, рассмотрим сначала ультрафиолетово-конечную теорию Виттена-Зайберга, а потом с помощью двойного скейлингова предела вернемся к асимптотически свободной теории. Для этого к чистой калибровочной $\mathcal{N}=2$ $4 \mathrm{D}$ суперсимметричной теории следует добавить гипермультиплет материи в присоединенном представлении с массой $m$. При $m=0$ возникает ультрафиолетово-конечная теория с $\mathcal{N}=4$ суперсимметрией и константой связи $\tau=i / g_{U V}^{2}+\theta / 2 \pi$. При $m \neq 0$ теория остается ультрафиолетово-конечной, но в ней появляется нетривиальный ренормгрупповой поток. $\mathcal{N}=2$ суперсимметричная теория Янга-Миллса восстанавливается в двойном скейлинговом пределе при $\tau \rightarrow i \infty, m \rightarrow \infty$ так, что комбинация $m^{N} e^{2 \pi i \tau} \equiv \Lambda^{N}$ остается конечной. В контексте теории Виттена-Зайберга это отвечает вырож дению тора с комплексной структурой $\tau$ в пределе $\tau \rightarrow \infty$ в цилиндр. Соответствуюшая интегрируемая система - эллиптическая модель Калоджеро-Мозера с константой связи $m$ - в этом пределе переходит в цепочку Тоды [39].

С точки зрения бранной картины (рис.4) это означает, что сначала надо заменить цилиндры торами с модулем $\tau$. Несвязанные торы будут буквально отвечать нулевому значению параметра $m$, в то время как ненулевое $m$ означает, что скалярное поле $\Phi$ обладает нетривиальными граничными условиями или является сечением нетривиального (голоморфного) расслоения. Другими словами, при склеивании цилиндров в торы поля приобретают некоторый "скачок", а в уравнении (103) на торе появляется нетривиальная правая часть, которая не исчезает и в двойном скейлинговом пределе.

Это означает, что на торе нельзя выбрать калибровку $\bar{A} \equiv A_{6}+i A_{9}=0$ и самое большее, что можно сделать калибровочным преобразованием, - это привести $\bar{A}$ к диагональному виду. Тогда соответствуюшее уравнение (103) преврашается в частный случай (53):

$$
\bar{\partial} \Phi^{i j}+\left(q_{i}-q_{j}\right) \Phi^{i j}=m\left(1-\delta^{i j}\right) \delta\left(z-z_{0}\right),
$$

так что

$$
\Phi^{i j}(z)=p_{i} \delta^{i j}+m\left(1-\delta^{i j}\right) e^{\left(q_{i}-q_{j}\right)(z-\bar{z})} \frac{\theta_{*}\left(z-z_{0}+\frac{\operatorname{Im} \tau}{\pi}\left(q_{i}-q_{j}\right)\right) \theta_{*}^{\prime}(0)}{\theta_{*}\left(z-z_{0}\right) \theta_{*}\left(\frac{\operatorname{Im} \tau}{\pi}\left(q_{i}-q_{j}\right)\right)} .
$$


Чтобы сравнить (105) с каноническим оператором Лакса эллиптической модели Калоджеро-Мозера [40], следует, как и раньше, сделать калибровочное преобразование

$$
\begin{aligned}
\Phi^{i j}(z) & \rightarrow\left(U^{-1} \Phi U\right)^{i j}(z)= \\
& =p_{i} \delta^{i j}+m\left(1-\delta^{i j}\right) e^{\left(q_{i}-q_{j}\right) z} \frac{\theta_{*}\left(z-z_{0}+\frac{\operatorname{Im} \tau}{\pi}\left(q_{i}-q_{j}\right)\right) \theta_{*}^{\prime}(0)}{\theta_{*}\left(z-z_{0}\right) \theta_{*}\left(\frac{\operatorname{Im} \tau}{\pi}\left(q_{i}-q_{j}\right)\right)},
\end{aligned}
$$

при этом явная зависимость от $\bar{z}$ пропадает, но $\Phi(z)$ преврашается в неоднозначную функцию или сечение нетривиального расслоения на торе. Оператор Лакса цепочки Тоды возникает из (106) в двойном скейлинговом пределе [39].

Безусловно, наложить нетривиальные граничные условия можно непосредственно в конфигурации рис. 4 (без дополнительных компактификаций и декомпактификаций измерения $\left.x^{6}\right)$. При этом возникает голоморфное уравнение Хитчина непосредственно на цилиндре [27] с дельта-функциями в обеих бесконечно удаленных точках

$$
\bar{\partial} \Phi=M_{\infty} \delta\left(P_{\infty}\right)-M_{0} \delta\left(P_{0}\right),
$$

а точный вид источников $M_{\infty}$ и $M_{0}$ является следствием эффективного взаимодействия в данном пределе лишь соседних бран.

Таким образом, следуя [37], мы получили аналитический вид кривой (101) "из первопринципов". Следуюший шаг - вывести эффективное действие низкоэнергетической $4 \mathrm{D}$ теории. Поскольку эффективное действие на мировом объеме браны не квадратично, для этого необходимо выполнить нетривиальное интегрирование по полям, которые становятся массивными благодаря эффекту Хиггса, более того, при этом возникают непертурбативные эффекты. Тем самым это более или менее эквивалентно первоначальной проблеме теории Виттена-Зайберга без каких-либо сушественных упрошений. Как уже было сказано в п. 2.3, с точки зрения интегрируемых систем это является задачей так называемого метода усреднения Боголюбова-Уизема, техника которого пока еше не вполне развита. Замечательно, что несмотря на эти проблемы результат процедуры усреднения может быть эффективно описан в терминах интегралов по периодам на римановых поверхностях, т.е. на языке препотенциалов (или уиземовских тау-функций), которые в определенном смысле "дуальны" системам Хитчина.

Это в точности соответствует тому, что происходит в теории Виттена-Зайберга: в отсутствие конкретного способа вывести эффективное действие относительно легко предложить анзац для его вида. Другими словами, несмотря на то что вакуумная конфигурация точно определяется в картине IIB, это представление не является достаточным для вывода эффективного действия (по крайней мере, это не очевидно). Однако согласно [5] такая задача может быть решена в "дуальной" картине IIA, где вместо 6D суперсимметричной теории Янга-Миллса на мировом объеме 5-браны возникает теория самодуальной 2-формы $C=\left\{C_{M N}\right\}, d C=* d C$. Это означает, что вместо открытых струн, как на рис. 2 , взаимодействие осушествляется "открытыми" мембранами.

Важным отличием от теории типа IIB является то, что теория 2-форм существенно абелева. Даже если ввести матрицы $C_{M N}^{i j}$ в присоединенном представлении $S U(N)$, 
отвечающие мембранам, натянутым между $i$-м и $j$-м цилиндрами, в теории не может возникнуть неабелево взаимодействие, так как это нарушило бы калибровочную инвариантность. В такой теории могут появиться лишь члены "неминимального" вида типа $\operatorname{Tr}(d C)^{4}$, т.е. зависящие от напряженности $C$. Однако такие члены содержат больше производных (старшие степени импульсов) и несушественны в низкоэнергетических эффективных действиях. Абелева природа теории 2-форм означает, что описание оператора Лакса (вакуумной матрицы скалярных полей супермультиплета, описывающих поперечные флуктуации 5-браны) и вывод вида кривой в картине IIA не являются легкой задачей. Благодаря квадратичности эффективного действия на (плоской) 5-бране,

$$
\int_{d^{6} x}|d C|^{2}+\text { суперсимметричные члены, }
$$

в этом представлении не возникает поправок к виду эффективного 4D действия, если уже задана кривая $\Sigma$. Достаточно рассмотреть размерную редукцию выражения (108) из $6 \mathrm{D}$ в 4D [5]. Эта редукция подразумевает, что для 2-формы $C$ сушествует разложение

$$
C_{\mu z}=\sum_{i=1}^{N-1}\left(A_{\mu}^{i}(x) d \omega_{i}(z)+\bar{A}_{\mu}^{i}(x) d \bar{\omega}_{i}(\bar{z})\right),
$$

где $d \omega_{i}$ - канонические голоморфные 1-дифференциалы $(37)$ на кривой $\Sigma^{13)}, d \bar{\omega}_{i}-$ комплексно сопряженные дифференциалы, а $A_{\mu}^{i}, \bar{A}_{\mu}^{i}$ зависят только от $4 \mathrm{D}$ координат $x=\left\{x^{0}, x^{1}, x^{2}, x^{3}\right\}$.

Если выбрать на кривой $\Sigma$ метрику так, что $* d \omega_{i}=-d \omega_{i}, * d \bar{\omega}_{i}=+d \bar{\omega}_{i}$, то самодуальность $C$ сводится к тому, что 1-формы $A$ и $\bar{A}$ в формуле (109) отвечают антисамодуальной и самодуальной компонентам 4D калибровочного поля с напряженностью $f=\left\{f_{\mu \nu}\right\}$

$$
d A^{i}=f^{i}-* f^{i}, \quad d \bar{A}^{i}=f^{i}+* f^{i} .
$$

Остается подставить (110) в (108) и использовать соотношения (38), (39), чтобы получить $T_{i j}$ - матрицу периодов поверхности $\Sigma$ (зависящую от вакуумных средних поперечных скалярных полей, поскольку мы уже определили вложение кривой $\Sigma$ в пространство $\left.\left(x^{4}, x^{5}, x^{6}, x^{10}\right)\right)$. В результате для $4 \mathrm{D}$ эффективного действия получаем

$$
\int_{d^{4} x} \operatorname{Im} T_{i j} f_{\mu \nu}^{i} f_{\mu \nu}^{j}+\text { суперсимметричные члены, }
$$

13) До взятия двойного скейлингова предела кривая $\Sigma$ является компактной римановой поверхностью рода $N$ (когда оба направления $x^{6}$ и $x^{10}$ компактны) и на ней существует $N$ голоморфных дифференциалов. Один из них, однако, при $z \rightarrow \infty$ приобретает полюс и поэтому не учитывается в (108). Кроме того, мы не учитываем компоненты 2-форм $C_{z} \bar{z}$ и $C_{\mu \nu}$, которые связаны друг с другом преобразованием самодуальности

$$
\partial_{\lambda} C_{z \bar{z}}=\frac{1}{\sqrt{g}} \epsilon_{\lambda \mu \nu \rho} \partial_{\rho} C_{\mu \nu}
$$

и отвечают с точки зрения 4-мерной теории (вешественному) скаляру. Тем самым имеются всего три комплексных скалярных поля, два из которых в данной конфигурации массивны. 
что совпадает с ответом Виттена-Зайберга с точностью до топологического $\theta$-члена, который может быть восстановлен чуть более аккуратным обращением с действием самодуальной 2-формы.

3.3. $\mathcal{N}=1$ спектральные кривые и солитоны. Таким образом, $\mathcal{N}=2$ спектральные кривые можно рассматривать как нетривиальный вакуум М-теории в $10+1$ (компактном) измерениях, склеив их из гиперповерхностей (бран), вложенных в 11D пространство посредством соотношений

$$
z=x^{6}+i x^{10}, \quad \lambda=x^{4}+i x^{5}, \quad \Lambda^{N} \operatorname{ch} z=P_{N}(\lambda) .
$$

Нарушение суперсимметрии $[10,41]$ предполагает предел безмассовых монополей, или, в частности,

$$
P_{N}(\lambda)=\Lambda^{N} \operatorname{ch} z, \quad \lambda=2 \operatorname{ch} \frac{z}{N} \equiv \xi+\xi^{-1} .
$$

При этом

$$
y^{2}=4 \Lambda^{2 N} \operatorname{sh}^{2} z=4 \Lambda^{2 N} \operatorname{sh}^{2} \frac{z}{N} Q^{2}(\lambda), \quad Q(\lambda) \equiv \frac{\operatorname{sh} z}{\operatorname{sh} \frac{z}{N}} .
$$

Согласно [41] к правилу вложения (112) следует добавить

$$
v=x^{7}+i x^{8}=\frac{\zeta}{\xi}=\zeta e^{-\frac{z}{N}}
$$

(вместо прежнего $v \equiv 0$ ). Мы продемонстрируем, что нарушение суперсимметрии до $\mathcal{N}=1$ (предел безмассовых монополей) отвечает солитонному пределу соответствуюшей конечнозонной интегрируемой системы, для чистой калибровочной теории - периодической цепочке Тоды. В частности, кривая (114) является "солитонной" кривой в цепочке Тоды, при этом полином $Q(\lambda)$ имеет корни

$$
Q(\lambda)=\prod_{j=1}^{N-1}\left(\lambda-2 \cos \frac{\pi j}{N}\right) .
$$

Мы построим соответствуюшие солитонные решения, используя явную форму функции БА (практически такое же рассуждение было использовано в [42]). Общий вид функции БА -

$$
\Psi_{n}^{( \pm)}(\xi, t)=\xi^{n} \exp \left\{\sum_{k} t_{k}\left(\xi^{k}-\xi^{-k}\right)\right\} \frac{R_{n}^{( \pm)}(\xi, t)}{R(\xi)},
$$

где

$$
\begin{gathered}
R(\xi)=\prod_{s=1}^{N-1}\left(\xi-\gamma_{s}\right) \\
R_{n}^{( \pm)}(\xi, t)=\psi_{n}^{( \pm)}(t) \prod_{s=1}^{N-1}\left(\xi-\mu_{s}(n, t)\right)=\sum_{k=0}^{N-1} r_{k}(n, t) \xi^{k}
\end{gathered}
$$


Уравнение Лакса цепочки Тоды (подробнее см., например, в [38])

$$
\begin{gathered}
\lambda \Psi_{n}=C_{n+1} \Psi_{n+1}+p_{n} \Psi_{n}+C_{n} \Psi_{n-1}, \\
C_{n} \equiv e^{\frac{1}{2}\left(q_{n}-q_{n-1}\right)}, \quad \lambda=\xi+\frac{1}{\xi},
\end{gathered}
$$

приводит к соотношениям

$$
\begin{aligned}
r_{0}(n)-C_{n} r_{0}(n-1) & =0, \\
r_{1}(n)-C_{n} r_{1}(n-1)-p_{n} r_{0}(n) & =0,
\end{aligned}
$$

т.е., в частности, к

$$
r_{0}(n)=C_{n} r_{0}(n-1)=\cdots=e^{\frac{1}{2}\left(q_{n}-q_{0}\right)} r_{0}(0) \sim e^{\frac{1}{2} q_{n}} .
$$

Для решений, являюшихся вырождением $N$-периодической цепочки Тоды, следует также наложить "условия склейки"

$$
\Psi_{n}\left(\xi_{j}\right)=\Psi_{n}\left(\frac{1}{\xi_{j}}\right), \quad j=1, \ldots, N-1,
$$

которые означают, что функция БА “помнит” о своем происхождении с римановой поверхности рода $N-1$ - каждая пара точек $\xi_{j}, 1 / \xi_{j}$ отвечает вырожденной ручке. Условие (122) вместе с явной формой (117) и $\Psi_{n+N}=w \Psi_{n}$ приводят к

$$
w=\xi^{N}, \quad \xi_{j}^{2 N}=1,
$$

т.e.

$$
\xi_{j}=e^{\frac{i \pi j}{N}}
$$

где индекс $j$ принимает значения $1, \ldots, N-1$, так как

$$
\phi_{j}=\xi_{j}+\frac{1}{\xi_{j}}=2 \cos \frac{\pi j}{N}=\phi_{2 N-j}
$$

Перепишем (122) в виде

$$
\begin{gathered}
\frac{R_{n}\left(\frac{1}{\xi_{j}}\right)}{R_{n}\left(\xi_{j}\right)}=\prod_{k=1}^{N-1} \frac{\xi_{j}^{-1}-\mu_{k}(n, t)}{\xi_{j}-\mu_{k}(n, t)}=\exp \left\{\frac{2 \pi i n j}{N}+4 i \sum_{l} t_{l} \sin \frac{\pi j l}{N}+Z_{j}(\gamma)\right\}, \\
Z_{j}(\gamma) \equiv \sum_{s=1}^{N-1} \ln \frac{\xi_{j}-\gamma_{s}}{1-\xi_{j} \gamma_{s}}, \quad j=1, \ldots, N-1,
\end{gathered}
$$

т.е. в виде системы линейных уравнений на коэффициенты $r_{k}(n, t)$ полинома $R_{n}(\xi, t)$

$$
\begin{gathered}
e^{\frac{i \Phi_{j}(n, t)}{2}} \sum_{k=1}^{N-1} \sin \left(\frac{\pi j k}{N}+\frac{\Phi_{j}(n, t)}{2}\right) r_{k}=0, \\
\Phi_{j}(n, t) \equiv \frac{2 \pi n j}{N}+4 \sum_{l} t_{l} \sin \frac{\pi j l}{N}-i Z_{j}(\gamma),
\end{gathered}
$$

2 Теоретическая и математическая физика, т. 121, № 2, 1999 г. 
которые тривиально решаются. Простейшая функция БА в случае $N=2$ имеет вид

$$
\Psi_{n}=\xi^{n} \phi_{n} \frac{\xi-\mu(n)}{\xi-\gamma}, \quad n=0,1,
$$

а система (126) приводит к уравнению

$$
\frac{1-i \mu(n)}{i-\mu(n)}=e^{i \pi n+4 i t+Z}, \quad n=0,1
$$

с решением

$$
m_{1} \equiv-i \mu_{1}=\frac{1}{m_{0}}=C=\operatorname{ch} \frac{\phi}{2}, \quad \phi=4 i t+Z+i \frac{\pi}{2} .
$$

Солитонное решение цепочки Тоды определяется соотношениями

$$
\begin{gathered}
C \equiv C_{1}=e^{\frac{1}{2}\left(q_{1}-q_{0}\right)} \equiv e^{\frac{q}{2}}=\frac{\psi_{1}}{\psi_{0}} \\
p=\mu_{1}-\mu_{0}, \quad \frac{\mu_{0}}{\mu_{1}}=C^{2}, \quad C^{2}=1+p \mu_{0},
\end{gathered}
$$

приводящими, в частности, к

$$
\operatorname{sh} \frac{q}{2} \operatorname{sh} \phi=1
$$

Другой способ убедиться, что нарушение суперсимметрии до $\mathcal{N}=1$ отвечает солитонному пределу, - изучить решения иерархии Уизема. Генерацию суперпотенциала в пределе $\mathcal{N}=1$ можно рассматривать как "включение" уиземовской динамики $\delta t_{k} \operatorname{Tr} \Phi^{k}$ в смысле [36] (см. п. 4.3). Для малых $\delta t_{k}$ это можно рассматривать как возмущение гладкого $\mathcal{N}=2$ решения или как вычисление некоторых корреляторов в $\mathcal{N}=2$ суперсимметричной калибровочной теории. В то же время собственно теория с $\mathcal{N}=1$ отвечает уиземовским решениям в пределе больших $t_{k}$, а асимптотика при больших $t_{k}$ уводит на гранищы пространства модулей или, другими словами, отвечает распаду гладких конечнозонных решений в солитоны [28] (см. также [17]). Подобные рассуждения оказываются вполне согласованными с D-бранными конструкциями $\mathcal{N}=1$ вакуумов в теории струн.

3.4. Компактификация в $(\mathbf{3}+\mathbf{1})$ D. Нарушение суперсимметрии можно естественно получить, изучая компактифицированные теории с нетривиальными граничными условиями. В контексте теории Виттена-Зайберга это дает возможность, например, рассматривать семейство теорий с присоединенной материей как различные пределы единой интегрируемой системы - эллиптической модели Руйсенарса-Шнайдера [43].

Рассмотрим теперь компактификацию $\mathcal{N}=2$ суперсимметричной теории Янга-Миллса с единственным векторным супермультиплетом в $(3+1) \mathrm{D}$ (с компактным направлением радиуса $R_{3} \equiv R$ ). Если потребовать периодических граничных условий для всех полей вдоль компактного направления, то полученная теория будет (в смысле 3 -мерной теории) $\mathcal{N}=4$ суперсимметричной. Если же потребовать [43]

$$
\phi(x+R)=e^{i \epsilon} \phi(x)
$$


для половины полей, то компактифицированная теория будет обладать лишш $\mathcal{N}=23 \mathrm{D}$ суперсимметрией (т.е. $\mathcal{N}=1$ в смысле 4-мерной теории), или суперсимметрия будет частично нарушена граничными условиями.

Рассмотрим случай $\mathcal{N}=2$ 4D векторного супермультиплета в присоединенном представлении, состоящего из $\mathcal{N}=14 \mathrm{D}$ векторного мультиплета $\left(A_{\mu}, \psi\right)$ и $\mathcal{N}=14 \mathrm{D}$ скалярного мультиплета $(\phi, \chi)$, где $\psi$ и $\chi$ - два комплексных вейлевских спинора. Потребуем, чтобы последний из них при сдвиге вдоль компактного направления умножался на фазу (133), это означает, в частности, что он получает массу $\epsilon / R . \mathcal{N}=14 \mathrm{D}$ векторный мультиплет остается безмассовым и с точки зрения 3 -мерной теории становится $\mathcal{N}=2$ супермультиплетом $\left(A_{i}, \psi, q / R\right)$, где $i=0,1,2, q=R A_{3}$, а $\psi$ - 3D комплексный спинор.

В отличие от $\mathcal{N}=4$ суперсимметрии в трехмерии, в $\mathcal{N}=2$ суперсимметричной теории может генерироваться суперпотенциал [44]. Если ввести переменные $\Phi_{i}=$ $T_{i j}(\phi) q_{j}+\gamma_{i}$, где $q_{i}-$ собственные значения матрицы монодромии $\oint A_{3}$, a $\gamma_{i}$ - дуальные фотоны в трехмерии $A_{i}=\epsilon_{i j k} \partial_{j} \gamma_{k}$, то суперпотенциал принимает вид $[45,46]$

$$
W(\Phi)=\sum_{i=1}^{N-1} e^{\Phi_{i+1}-\Phi_{i}}+e^{\Phi_{1}-\Phi_{N}}
$$

и индуцируется $(3+1) \mathrm{D}$ инстантонными вкладами (см., например, работу [47] и приведенную в ней библиографию). Члены в сумме, отвечающие простым корням, определяются обычными трехмерными инстантонами ("БПС монополями"), приводящими к суперпотенциалу открытой цепочки Тоды, в то время как последний член (отрицательньй корень) появляется лишь в $(3+1)$ измерениях и может рассматриваться как вклад 4D инстантона (калорона).

В пределе слабой связи этот член обрашается в нуль, приводя к открытой цепочке Тоды [45]. Например, в вырожденном $S L(2)$ “лиувиллевском пределе" решение имеет особенно простой вид. Действительно, из

$$
\Lambda^{2} e^{2 q}=u-p^{2}=u-\left(\frac{d q}{d t}\right)^{2}
$$

и равенства $X=\Lambda^{2} e^{2 q}$ следует, что

$$
d t=\frac{d q}{\sqrt{u-\Lambda^{2} e^{2 q}}}=\frac{d X}{2 X \sqrt{u-X}}=\frac{d p}{p^{2}-u},
$$

и интегрирование приводит к

$$
p=\sqrt{u} \frac{1+e^{\vartheta}}{1-e^{\vartheta}}=\sqrt{u} \frac{1+e^{2 \sqrt{u} t}}{1-e^{2 \sqrt{u} t}}=-\sqrt{u} \operatorname{ch} \sqrt{u} t,
$$

так что

$$
\Lambda^{2} e^{2 q}=X=2 u \frac{e^{\vartheta}}{1-e^{\vartheta}}=2 u \sum_{n=1}^{\infty} e^{n \vartheta}
$$

Если $\vartheta \ll 1$, то $e^{2 q} \sim e^{\vartheta}$, и мы получаем классический или “затравочный” суперпотенциал. Таким образом, действительно видно, что квантование, по крайней мере в смысле 
построения эффективных действий, имеет вид канонического преобразования для некоторой интегрируемой системы ${ }^{14)}$.

\section{4. ИНТЕГРИРУЕМЫЕ УРАВНЕНИЯ В ТОПОЛОГИЧЕСКИХ СТРУННЫХ МОДЕЛЯХ И ТЕОРИИ ВИТТЕНА-ЗАЙБЕРГА}

Перейдем, наконец, непосредственно к структурам интегрируемых систем, возникающих в теории струн. Прежде чем обратиться к более или менее реалистичным ("физическим") теориям Виттена-Зайберга, мы рассмотрим простейшие из существующих струнных моделей - теории без материи или $2 \mathrm{D}$ теории квантовой гравитации, а также топологические струнные модели. Для этого класса теорий производяшая функция всех корреляторов является логарифмом тау-функции интегрируемой системы:

$$
\mathcal{F}(\mathbf{T})=\ln \mathcal{T}(\mathbf{T}),
$$

т.е. спектр операторов, отвечающих физическим величинам, параметризуется набором времен некоторой интегрируемой иерархии $\mathbf{T} \equiv\left\{T_{k}\right\}$, а корреляционные функции выражаются через решения этой иерархии нелинейных дифференциальных уравнений производные производяшей функции (139). Другими словами, коэффициенты разложения (139) по временам $\mathbf{T}$ задают значения соответствующих корреляционных функций. В случае теории струн начальные или граничные условия могут быть сформулированы как обращение в нуль производящей функции $\mathcal{F}$ действием некоторого дифференциального оператора первого порядка:

$$
L \mathcal{F}=0 .
$$

Это условие называется струнным уравнением. Сформулированноев терминах оператора Лакса струнное уравнение имеет вид "квантования" конечнозонного условия (31).

4.1. Топологические теории: препотенциал и уравнения Виттена-Дийкграфа-Верлинде-Верлинде. Простейшие струнные модели возникают, если рассматривать теории на мировом листе "без возбуждений" или, точнее, только с вакуумными состояниями в спектре. С точки зрения двумерного лагранжиана наиболее естественным выбором являются модели с $2 \mathrm{D}$ твистованной $\mathcal{N}=2$ суперсимметрией на мировом листе [48] (см. также [49]). Результат этой конструкции может быть сформулирован следуюшим образом.

- Сушествует (обычно конечный) набор так называемых примарных полей $\Phi_{\alpha}$ и соответствуюших им "вакуумных" состояний $|\alpha\rangle$.

- Среди этих состояний обычно имеется "тривиальный” (или нейтральный) вакуум $|0\rangle$ и соответствующий ему тождественный или единичный оператор $\Phi_{0}=P$, которому отвечает "прокол" без вставки в соответствующей отмеченной точке на мировом листе.

- “Лагранжиан" топологической теории определяется "пропагатором"

$$
\eta_{\alpha \beta}=\left\langle\Phi_{\alpha} \Phi_{\beta}\right\rangle \equiv\left\langle\Phi_{\alpha} \Phi_{\beta} P\right\rangle
$$

\footnotetext{
14) Для рассматриваемого случая цепочки Тоды подробное обсуждение и некоторую классификацию интегрируемых систем в контексте теории Виттена-Зайберга можно найти, например, в $[38,43]$.
} 
и вершинами, которые можно объединить в некоторую функцию $F\left(\left\{t_{\alpha}\right\}\right)$,

$$
\Gamma_{\alpha_{1} \ldots \alpha_{n}}=\left\langle\Phi_{\alpha_{1}} \otimes \cdots \otimes \Phi_{\alpha_{n}}\right\rangle=\left.\partial_{\alpha_{1}} \ldots \partial_{\alpha_{n}} F(t)\right|_{t=0} .
$$

Определение топологической теории подразумевает, что она не зависит от метрики на мировом листе. В частности, это означает, что корреляторы не зависят явно от положения операторов на мировом листе. Мы используем два разных обозначения для произведения операторов под знаком корреляционной функции: $\Phi_{\alpha} \Phi_{\beta} \equiv \Phi_{\alpha} \cdot \Phi_{\beta}$ и $\Phi_{\alpha} \otimes \Phi_{\beta}$. Первый случай отвечает ситуации, когда операторы $\Phi_{\alpha}=\Phi_{\alpha}(x)$ и $\Phi_{\beta}=\Phi_{\beta}(y)$ расположены в разных точках $x \neq y$. Так как топологическая теория не зависит от метрики, то растяжением можно добиться, чтобы расстояние $\|x-y\|^{2}$ стало бесконечным, т.е. первое определение можно рассматривать как произведение операторов, расположенных далеко друг от друга. Согласно второму определению тензорное произведение $\Phi_{\alpha} \otimes \Phi_{\beta} \equiv \Phi_{\alpha}(x) \otimes \Phi_{\beta}(x)$ отвечает действию операторов в одной и той же точке $x$, зависимость от которой можно опустить по той же причине, что и раньше - коррелятор с "композитным" полем $\Phi_{\alpha} \otimes \Phi_{\beta}$ не зависит от положения этого поля $x$, так как теория не зависит от двумерной метрики.

Следует сделать два дополнительных замечания.

1. Формула (141) выражает двухточечный пропагатор $\eta_{\alpha \beta}$ через трехточечную функцию $\left\langle\Phi_{\alpha} \Phi_{\beta} P\right\rangle$ с дополнительно вставленным единичным оператором. Причина заключается в том, что с точки зрения теории струн пропагатор является корреляционной функцией на сфере - мировом листе простейшей топологии, а на сфере хорошо определены лишь корреляторы с по меньшей мере тремя отмеченными точками.

2. Для трехточечных корреляционных функций нет разницы между двумя типами произведений операторов, которые обсуждались выше, т.е. можно написать

$$
\left\langle\Phi_{\alpha} \Phi_{\beta} P\right\rangle=\left\langle\Phi_{\alpha} \otimes \Phi_{\beta} \otimes P\right\rangle=\left.\partial_{\alpha} \partial_{\beta} \partial_{0} F(t)\right|_{t=0} .
$$

Это верно даже для корреляционных функций, зависящих от источников или времен,

$$
\langle\langle\mathcal{O}\rangle\rangle \equiv\left\langle\mathcal{O} \otimes \exp \left\{\sum t_{\alpha} \Phi_{\alpha}\right\}\right\rangle,
$$

т.e.

$$
\begin{aligned}
\left\langle\left\langle\Phi_{\alpha} \otimes \Phi_{\beta} \otimes P\right\rangle\right\rangle & =\left\langle\Phi_{\alpha} \otimes \Phi_{\beta} \otimes P\right\rangle=\eta_{\alpha \beta}, \\
\left\langle\left\langle\Phi_{\alpha} \otimes \Phi_{\beta} \otimes \Phi_{\gamma}\right\rangle\right\rangle & =\left\langle\left\langle\Phi_{\alpha} \Phi_{\beta} \Phi_{\gamma}\right\rangle\right\rangle .
\end{aligned}
$$

Если принять данное определение топологической теории, то становится очевидно, что вся информация о ней заключена в единственной функции конечного числа переменных $F(t)$, которую обычно называют препотенциалом. В случае $2 \mathrm{D}$ топологических моделей эта функция должна удовлетворять условию $(145)^{15)}$, т.е.

$$
\partial_{\alpha} \partial_{\beta} \partial_{0} F(t)=\eta_{\alpha \beta}
$$

\footnotetext{
15) Это требование не является универсальным. Другими словами, в 2D топологических моделях оно появляется "случайно": в ситуации общего положения, например в теории Виттена-Зайберга, существует много примеров препотенциалов, которые не удовлетворяют этому условию.
} 
где $\eta_{\alpha \beta}$ - постоянная матрица. Главное условие на функцию $F(t)$ возникает при рассмотрении операторной алгебры, которая в топологических теориях обладает структурой коммутативного кольца

$$
\Phi_{\alpha} \cdot \Phi_{\beta}=C_{\alpha \beta}^{\gamma} \Phi_{\gamma}
$$

где $C_{\alpha \beta}^{\gamma}=C_{\alpha \beta}^{\gamma}(t)=C_{\beta \alpha}^{\gamma}(t)$-набор $\mathbb{C}$-числовых симметричных матриц. Применяя (147) к четырехточечной функции $\left\langle\left\langle\Phi_{\alpha} \Phi_{\beta} \Phi_{\sigma} \Phi_{\rho}\right\rangle\right\rangle\left(\right.$ но не к $\left.\left\langle\left\langle\Phi_{\alpha} \otimes \Phi_{\beta} \otimes \Phi_{\sigma} \otimes \Phi_{\rho}\right\rangle\right) !\right)$, получаем

$$
\left\langle\left\langle\Phi_{\alpha} \Phi_{\beta} \Phi_{\sigma} \Phi_{\rho}\right\rangle\right\rangle=C_{\alpha \beta}^{\gamma}\left\langle\left\langle\Phi_{\gamma} \Phi_{\sigma} \Phi_{\rho}\right\rangle\right\rangle=C_{\alpha \sigma}^{\gamma}\left\langle\left\langle\Phi_{\gamma} \Phi_{\beta} \Phi_{\rho}\right\rangle\right\rangle
$$

или просто [49]

$$
C_{\alpha \beta}^{\gamma} C_{\gamma \sigma}^{\rho}=C_{\alpha \sigma}^{\gamma} C_{\gamma \beta}^{\rho},
$$

где индексы поднимаются или опускаются метрикой - пропагатором $\eta_{\alpha \beta}$. Формулы (149) можно рассматривать как систему нелинейных дифференциальных уравнений на функцию $F(t)$, называемых уравнениями Виттена-Дийкграфа-Верлинде-Верлинде (ВДВВ).

Интегрируемая структура, проявляющаяся при формулировке $2 \mathrm{D}$ топологических моделей, может быть элегантно записана в виде условий нулевой кривизны $[50,34]$

$$
\left[\nabla_{\alpha}, \nabla_{\beta}\right]=0, \quad \nabla_{\alpha}=\frac{\partial}{\partial t_{\alpha}}-\frac{1}{z} \mathbf{C}_{\alpha}, \quad \mathbf{C}_{\alpha} \equiv\left\|\mathbf{C}_{\alpha}\right\|_{\beta}^{\gamma}=C_{\alpha \beta}^{\gamma} .
$$

Благодаря зависимости от спектрального параметра z уравнение (150) приводит к двум независимым условиям

$$
\begin{aligned}
\frac{1}{z^{2}}\left[\mathbf{C}_{\alpha}, \mathbf{C}_{\beta}\right] & =0, \\
\frac{1}{z}\left(\partial_{\alpha} C_{\beta \gamma}^{\delta}-\partial_{\beta} C_{\alpha \gamma}^{\delta}\right) & =0 .
\end{aligned}
$$

Используя тот факт, что метрика $\eta$ (146) - постоянная матрица, формулу (152) можно переписать в виде

$$
\partial_{[\beta} F_{\alpha] \gamma \delta}=0
$$

что означает (по крайней мере, локально), что $F_{\alpha \beta \gamma}=\partial_{\alpha} T_{\beta \gamma}$ или, принимая во внимание, что $F_{\alpha \beta \gamma}$ симметричны по всем индексам,

$$
F_{\alpha \beta \gamma}=\partial_{\alpha} \partial_{\beta} \partial_{\gamma} F
$$

Рассмотрим несколько явных примеров алгебры (147).

1. Кольцо полиномов. Это пример так называемых топологических теорий Гинзбурга-Ландау, которые определяются полиномиальным суперпотенциалом в обшем случае от нескольких комплексных переменных. В простейшей ситуации это просто полином $W(X)$ комплексной переменной $X$, а кольцо (147) задается по правилу

$$
P_{i}(X) P_{j}(X)=C_{i j}^{k} P_{k}(X) \quad\left(\bmod W^{\prime}(X)\right)
$$


и является не чем иным, как фактором кольца всех полиномов по идеалу $W^{\prime}(X)=0$, т.е. $P_{i}(X)$ задают некоторый базис в $\mathbf{C}[X] / W^{\prime}(X)$. При этом автоматически коммутируют матрицы $\mathbf{C}_{i}$ и $\mathbf{C}_{j}$ как матрицы структурных констант (коммутативного) кольца:

$$
\left[\mathbf{C}_{i}, \mathbf{C}_{j}\right]=0 .
$$

Для топологических моделей Гинзбурга-Ландау препотенциал можно восстановить по так называемой формуле вычетов

$$
F_{i j k}=\left.\operatorname{Res}\right|_{W^{\prime}=0} \frac{P_{i} P_{j} P_{k}}{W^{\prime}} .
$$

Проверим эту конструкцию на примере топологической модели с суперпотенциалом

$$
W^{\prime}(X)=X^{3}-q
$$

(следует выбрать $q \neq 0$, тогда корни $W^{\prime}(X)$ различны (случай общего положения), чтобы матрицы $F_{1}$ и $F_{2}$ были невырожденны). В базисе $P_{i}=X^{i-1}, i=1,2,3$, из (157) легко получить [51]

$$
F_{0}=\left(\begin{array}{lll}
0 & 0 & 1 \\
0 & 1 & 0 \\
1 & 0 & 0
\end{array}\right), \quad F_{1}=\left(\begin{array}{lll}
0 & 1 & 0 \\
1 & 0 & 0 \\
0 & 0 & q
\end{array}\right), \quad F_{2}=\left(\begin{array}{lll}
1 & 0 & 0 \\
0 & 0 & q \\
0 & q & 0
\end{array}\right) .
$$

Соответствующий препотенциал имеет вид

$$
F=\frac{1}{2} t_{0} t_{1}^{2}+\frac{1}{2} t_{0}^{2} t_{2}+\frac{q}{2} t_{1} t_{2}^{2}
$$

а для обратных матриц имеем $F_{i}^{-1}(q)=F_{i}(1 / q)$. Чтобы сократить вычисления, полезно заметить, что из-за симметричности матриц $F_{i}$ соотношения $(149)$ эквивалентны тому, что все матрицы

$$
U_{i k j}=F_{i} F_{k}^{-1} F_{j}, \quad U_{i k j}=U_{i k j}^{\mathrm{tr}},
$$

также симметричны. Тривиальной проверкой легко убедиться, что это в самом деле так:

$$
\begin{aligned}
& U_{102}=F_{1} F_{0}^{-1} F_{2}=q I, \quad U_{201}=q I, \\
& U_{012}=F_{1}(q), \quad U_{210}=F_{1}(1 / q), \\
& U_{021}=U_{120}=\left(\begin{array}{ccc}
1 / q & 0 & 0 \\
0 & 0 & 1 \\
0 & 1 & 0
\end{array}\right) .
\end{aligned}
$$

Мы использовали простейший (недеформированный) базис полиномов $P_{i}(X)=$ $X^{i-1}$. Для суперпотенциала обшего положения $W(X)=X^{p}+\sum_{k=0}^{p-2} v_{k} X^{k}$ более естественным базисом является

$$
P_{k}(X) \equiv \frac{\partial W}{\partial t_{k}}=\frac{\partial}{\partial \lambda} W_{+}^{\frac{k}{p}}
$$

где

$$
t_{k}=-\left.\frac{p}{k(p-k)} \operatorname{Res}\right|_{\infty} W^{1-\frac{k}{p}} d \lambda .
$$

Легко проверить, что, продифференцировав (164) и подставив результат в (163), получим тождество. 
2. Квантовые когомологии. Другим примером являются топологические теории типа А, которые можно рассматривать как $\mathcal{N}=2$ 2D суперсимметричные сигма-модели на комплексных многообразиях, так что наблюдаемыми являются когомологии соответствующих многообразий, а инстантоны (голоморфные отображения) деформируют кольцо когомологий [52]. В простейшем случае сигма-моделей на $\mathbb{C} P^{1}$ пространство наблюдаемых двумерно: $\Phi_{0}=\mathbf{1} \in H^{(0)}$ и $\Phi_{1}=\omega \in H^{(2)}$, так что уравнения ВДВВ выполняются тривиально - эти уравнения всегда тривиальны в случае менее трех аргументов: любая алгебра (147) размерности 1 или 2 ассоциативна. Кольцо квантовых когомологий $\mathbb{C} P^{1}$ имеет вид ${ }^{16)}$

$$
\begin{aligned}
& \mathbf{1} \cdot \mathbf{1}=\mathbf{1}, \\
& \omega \cdot \mathbf{1}=\omega, \\
& \omega \cdot \omega=e^{t_{1}} \mathbf{1},
\end{aligned}
$$

а соответствующим препотенциалом является

$$
F=\frac{1}{2} t_{0}^{2} t_{1}+e^{t_{1}}
$$

так что вторые производные можно записать в виде

$$
F_{00}=t_{1}, \quad F_{01}=t_{0} t_{1}, \quad F_{11}=e^{t_{1}}
$$

а третьи образуют матрицы $F_{i} \equiv\left\|F_{i}\right\|_{j k}=F_{i j k}$ :

$$
F_{0}=\left(\begin{array}{ll}
0 & 1 \\
1 & 0
\end{array}\right), \quad F_{1}=\left(\begin{array}{cc}
1 & 0 \\
0 & e^{t_{1}}
\end{array}\right)
$$

даюшие структурные константы $C_{i}=F_{0}^{-1} F_{i}$ :

$$
C_{0}=\left(\begin{array}{ll}
1 & 0 \\
0 & 1
\end{array}\right), \quad C_{1}=\left(\begin{array}{cc}
0 & e^{t_{1}} \\
1 & 0
\end{array}\right)
$$

Рассмотрим теперь нетривиальное обобшение предыдушего примера - квантовые когомологии $\mathbb{C} P^{2}[53]$. В этом случае препотенциал имеет вид

$$
F=\frac{1}{2} t_{0} t_{1}^{2}+\frac{1}{2} t_{0}^{2} t_{2}+\sum_{n=1}^{\infty} \frac{N_{n} t_{2}^{3 n-1}}{(3 n-1) !} e^{n t_{1}}
$$

а соответствующие матрицы -

$$
F_{0}=\left(\begin{array}{lll}
0 & 0 & 1 \\
0 & 1 & 0 \\
1 & 0 & 0
\end{array}\right), \quad F_{1}=\left(\begin{array}{ccc}
0 & 1 & 0 \\
1 & F_{111} & F_{112} \\
0 & F_{112} & F_{122}
\end{array}\right), \quad F_{2}=\left(\begin{array}{ccc}
1 & 0 & 0 \\
0 & F_{112} & F_{122} \\
0 & F_{122} & F_{222}
\end{array}\right),
$$

${ }^{16)}$ В недеформированном кольце когомологий $\mathbb{C} P^{1}$ первые два соотношения те же самые, а вместо третьего выполняется $\omega \cdot \omega=0$. 
где

$$
\begin{aligned}
& F_{111}=\sum_{n} \frac{n^{3} N_{n}}{(3 n-1) !} t_{2}^{3 n-1} e^{n t_{1}}, \\
& F_{112}=\sum_{n} \frac{n^{2} N_{n}}{(3 n-2) !} t_{2}^{3 n-2} e^{n t_{1}}, \\
& F_{122}=\sum_{n} \frac{n N_{n}}{(3 n-3) !} t_{2}^{3 n-3} e^{n t_{1}}, \\
& F_{222}=\sum_{n} \frac{N_{n}}{(3 n-4) !} t_{2}^{3 n-4} e^{n t_{1}} .
\end{aligned}
$$

Легко проверить, что уравнения (149) эквивалентны условию

$$
F_{222}=F_{112}^{2}-F_{111} F_{122},
$$

которое приводит к рекуррентным формулам для $N_{n}$ - чисел рациональных кривых степени $n$ с $3 n-1$ неподвижными точками в $\mathbb{C} P^{2}[53]$ :

$$
\frac{N_{n}}{(3 n-4) !}=\sum_{a+b=n} \frac{a^{2} b(3 b-1) b(2 a-b)}{(3 a-1) !(3 b-1) !} N_{a} N_{b}
$$

Например, $N_{2}=N_{1}^{2}, N_{3}=12 N_{1} N_{2}=12 N_{1}^{3}, \ldots$.

Другое интересное обобшение кольца квантовых когомологий $\mathbb{C} P^{1}$ связано непосредственно с интегрируемыми системами тодовского типа. Легко заметить, что основное соотношение (165) может быть переписано как

$$
h_{\mathrm{L}}=p^{2}-e^{q}=0,
$$

т.е. в виде равенства нулю гамильтониана уравнения Лиувилля, если формально обозначить $\omega \equiv p$ и $t_{1} \equiv q$, т.е. отождествить импульс с представителем класса когомологий, а координату - с параметром деформации алгебры (147). Недеформированное кольцо отвечает “свободной частице”, а деформация приводит к простейшей (открытой!) модели Тоды.

Это совпадение не случайно. Согласно [54] обобщения (175) на открытые цепочки Тоды со многими частицами действительно описьвают деформации колец когомологий для класса многообразий полных флагов. Импульсы частиц $p_{i}$ отождествляются с представителями $\omega_{i} \in H^{(2)}$, а координаты $q_{i+1, i}-$ с параметрами деформации $t_{i}{ }^{17)}$. Обшее кольцо когомологий полных флагов, таким образом, может быть описано уравнением

$$
\operatorname{det}(\lambda-\mathcal{L})=0
$$

\footnotetext{
17) В используемых обозначениях импульсы нумеруются векторами фундаментального представления, т.е. $\sum_{i} p_{i}=0$, в то время как относительные координаты $q_{i+1, i} \equiv q_{i+1}-q_{i}-$ простыми корнями.
} 
где $\mathcal{L}$ - оператор Лакса открытой цепочки Тоды, или

$$
h_{k}(p, q)=0, \quad k=1, \ldots, \operatorname{rank} G .
$$

В первом нетривиальном примере $s l(3)$ цепочки Тоды формулы (176), (177) принимают вид

$$
\mathcal{L}_{s l(3)}=\left(\begin{array}{ccc}
p_{1} & e^{\frac{1}{2} q_{21}} & 0 \\
e^{\frac{1}{2} q_{21}} & p_{2} & e^{\frac{1}{2} q_{32}} \\
0 & e^{\frac{1}{2} q_{32}} & p_{3}
\end{array}\right),
$$

т.е. обрашение в нуль гамильтонианов дает

$$
\begin{aligned}
& h_{1}=p_{1}+p_{2}+p_{3}=0, \\
& h_{2}=p_{1} p_{2}+p_{2} p_{3}+p_{1} p_{3}-e^{q_{21}}-e^{q_{32}}=0, \\
& h_{3}=p_{1} p_{2} p_{3}-p_{1} e^{q_{32}}-p_{3} e^{q_{21}}=0 .
\end{aligned}
$$

Решая первое (линейное) уравнение в (179) относительно $p_{2}$, можно отождествить это кольцо когомологий с кольцом полиномов двух переменных $\omega_{1} \equiv-p_{1}$ и $\omega_{2} \equiv p_{3}$ по модулю:

$$
\begin{aligned}
& \omega_{1} \omega_{2}=\omega_{1}^{2}+\omega_{2}^{2}+e^{t_{1}}+e^{t_{2}}, \\
& \omega_{1} \omega_{2}^{2}=\omega_{1}^{2} \omega_{2}-e^{t_{2}} \omega_{1}+e^{t_{1}} \omega_{2} .
\end{aligned}
$$

Это кольцо легко описать явно. Например, в базисе $1, \omega_{1}, \omega_{2}, \omega_{1}^{2}, \omega_{2}^{2}, \omega_{1}^{2} \omega_{2}$ алгебра (147) задается матрицами $C_{\alpha}$ размера $6 \times 6, \alpha=0,1, \ldots, 5$, имеюшими вид

$$
C_{1}=\mathbf{1}
$$

а также

$$
\begin{aligned}
C_{\omega_{1}} & =\left(\begin{array}{cccccc}
0 & 1 & 0 & 0 & 0 & 0 \\
0 & 0 & 0 & 1 & 0 & 0 \\
e^{t_{1}}+e^{t_{2}} & 0 & 0 & 1 & 1 & 0 \\
0 & -e^{t_{1}} & -e^{t_{1}} & 0 & 0 & 0 \\
0 & -e^{t_{2}} & e^{t_{1}} & 0 & 0 & 1 \\
-e^{t_{1}}\left(e^{t_{1}}+e^{t_{2}}\right) & 0 & 0 & -e^{t_{1}} & -2 e^{t_{1}} & 0
\end{array}\right), \\
C_{\omega_{2}} & =\left(\begin{array}{cccccc}
0 & 0 & 1 & 0 & 0 & 0 \\
e^{t_{1}}+e^{t_{2}} & 0 & 0 & 1 & 1 & 0 \\
0 & 0 & 0 & 0 & 1 & 0 \\
0 & 0 & 0 & 0 & 0 & 1 \\
0 & -e^{t_{2}} & -e^{t_{2}} & 0 & 0 & 0 \\
0 & 0 & 0 & -e^{t_{2}} & -e^{t_{1}} & 0
\end{array}\right)
\end{aligned}
$$

и т.д. Легко проверить, что эти матрицы коммутируют, т.е. удовлетворяют (151). 
3. Уравнения ассоциативности в теории Виттена-Зайберга [51]. Этот случай отличается от $2 \mathrm{D}$ топологических теорий отсутствием естественного выбора единичного оператора. Поэтому, несмотря на аналогию, а в некоторых случаях и полную эквивалентность алгебраической структуры, теория Виттена-Зайберга приводит к более общей форме и новым решениям уравнений ассоциативности, в частности, выходящим за рамки классификации [34].

Первым (и достаточно впечатляюшим) примером таких решений являются пертурбативные препотенциалы теории Виттена-Зайберга (20). Например, для $S U(N)$ калибровочной теории они имеют следующий вид:

$$
\mathcal{F}_{\text {pert }}=\frac{1}{2} \sum_{\substack{i<j \\ i, j=1}}^{N-1}\left(a_{i}-a_{j}\right)^{2} \ln \left(a_{i}-a_{j}\right)+\frac{1}{2} \sum_{i=1}^{N-1} a_{i}^{2} \ln a_{i} .
$$

Достаточно легко продемонстрировать явным вычислением (см. подробности доказательства в $[51,55])$, что препотенциал (184) удовлетворяет системе дифференциальных уравнений

$$
\mathcal{F}_{i} \mathcal{F}_{k}^{-1} \mathcal{F}_{j}=\mathcal{F}_{j} \mathcal{F}_{k}^{-1} \mathcal{F}_{i} \quad \forall i, j, k=1, \ldots, N-1,
$$

где $\mathcal{F}_{i}$ - матрица третьих производных

$$
\left(\mathcal{F}_{i}\right)_{m n}=\frac{\partial^{3} \mathcal{F}}{\partial a_{i} \partial a_{m} \partial a_{n}}
$$

или

$$
\mathcal{F}_{i} G^{-1} \mathcal{F}_{j}=\mathcal{F}_{j} G^{-1} \mathcal{F}_{i} \quad \forall i, j, k=1, \ldots, N-1,
$$

где $G=\sum_{k} g_{k} \mathcal{F}_{k}$ для любых $g_{k}$. Легко видеть, что в общем случае в отличие от $2 \mathrm{D}$ топологических моделей метрика $G$ не является постоянной ${ }^{18)}$.

Более того, уравнения (185) верны вне рамок теории возмушений для большинства препотенциалов Виттена-Зайберга (важным исключением является случай теории с нарушенной $\mathcal{N}=4$ суперсимметрией - эллиптическая модель Калоджеро-Мозера [55]). Проблема взаимосвязи уравнений (185) с уравнениями ВДВВ в топологической теории струн (149), (151) (или, точнее, вопрос о том, как уравнения ассоциативности в теории Виттена-Зайберга следуют из теории струн) до сих пор остается открытой.

18) Только для пертурбативного решения (184) существует более или менее естественный выбор постоянной метрики - в виде "бета-функции" при $g_{k}=a_{k}$ или

$$
\beta_{i j}=\frac{\partial T_{i j}}{\partial \ln \Lambda}=-\sum_{k} a_{k} \mathcal{F}_{i j k}
$$

которая совпадает с матрицей Картана $\beta_{i j}=K_{i j}$ для препотенциала (184). 
4.2. Топологические теории струн и $2 \mathrm{D}$ квантовая гравитация. Уравнение (150) содержит уже больше информации, чем просто формулировка топологической теории, данная в п. 4.1. Действительно, вспомогательная линейная задача [34]

$$
\nabla_{\alpha} \xi_{\beta}=\partial_{\alpha} \xi_{\beta}-\frac{1}{z} C_{\alpha \beta}^{\gamma} \xi_{\gamma}=0
$$

имеет решение $[34,54]$

$$
\xi_{\beta}=\sum_{n \geqslant 0} \frac{1}{z^{n+1}}\left\langle\left\langle\sigma_{n}\left(\Phi_{\beta}\right) \exp \left\{\sum t_{\alpha} \Phi_{\alpha}\right\}\right\rangle\right\rangle
$$

где операторы $\sigma_{n}\left(\Phi_{\alpha}\right)$ отвечают гравитационным потомкам операторов топологической теории.

В простейшем случае тривиальной топологической теории с единственным "тождественным" оператором $\Phi_{0} \equiv P$ решение задачи (189) есть просто экспонента $\xi_{P}=$ $e^{t_{0} / z}$. Однако производяшая функция гравитационных потомков $\sigma_{n} \equiv \sigma_{n}(P)$ уже нетривиальна и “считает" индексы пересечений на пространствах модулей комплексных кривых с отмеченными точками. Эта производящая функция была вычислена в [56] и представлена в форме матричного интеграла

$$
Z=\sum_{\left\{n_{i}\right\}} \prod_{i} \frac{T_{i}^{n_{i}}}{n_{i} !}\left\langle\sigma_{i}^{n_{i}}\right\rangle=\frac{\int D X e^{-\operatorname{Tr} M X^{2}-\operatorname{Tr} \frac{X^{3}}{3}}}{\int D X e^{-\operatorname{Tr} M X^{2}}}
$$

где операторы $\sigma_{k}$ отвечают временам $T_{2 k+1}=[1 /(2 k+1)] \operatorname{Tr} M^{-2 k-1}, k=0,1,2, \ldots$ Переписывая (191) в виде

$$
Z=e^{-\frac{2}{3} \operatorname{Tr} M^{3}} \operatorname{det}(M \otimes 1+1 \otimes M)^{-\frac{1}{2}} \int D X e^{-\operatorname{Tr} \frac{X^{3}}{3}+\operatorname{Tr} M^{2} X}=\frac{\operatorname{det}_{i j} \varphi_{i}\left(m_{j}\right)}{\prod_{i<j}\left(m_{i}-m_{j}\right)}
$$

где $m_{i}$ - собственные значения матрицы $M$, a

$$
\varphi_{i}(m)=\sqrt{2 m} e^{-\frac{2}{3} m^{3}} \int d x x^{i-1} e^{-\frac{x^{3}}{3}+m^{2} x}
$$

можно заметить [57], что статсумма $Z=\mathcal{T}(\mathbf{T})$ является не чем иным, как тау-функцией иерархии Кд $\Phi$, удовлетворяющей дополнительным (линейным) уравнениям - условиям Вирасоро или струнным уравнениям $[58,59]$

$$
\begin{gathered}
L_{n} Z=0, \quad n \geqslant-1, \\
L_{n}=\frac{1}{2} \sum_{\substack{k>\delta_{n+1,0} \\
k(\text { odd })}} k T_{k} \frac{\partial}{\partial T_{k+2 n}}+\frac{1}{4} \sum_{\substack{a+b=2 n \\
a, b>0(\text { odd })}} \frac{\partial^{2}}{\partial T_{a} \partial T_{b}}+ \\
+\delta_{n+1,0} \cdot \frac{T_{1}^{2}}{4}+\delta_{n, 0} \cdot \frac{1}{16}-\frac{\partial}{\partial T_{2 n+3}}
\end{gathered}
$$


Подобное утверждение практически без изменений может быть сформулировано для топологических моделей Ландау-Гинзбурга (взаимодействующих с $2 \mathrm{D}$ топологической гравитацией) серии $A_{p}$, т.е. с суперпотенциалом

$$
W(X)=X^{p}+\sum_{j=0}^{p-2} v_{j} X^{j}
$$

Для статсумм этих теорий существует представление [57]

$$
\begin{gathered}
Z=\frac{e^{-V(M)+M W(M)}}{\operatorname{det}\left(W^{\prime}(M) \otimes 1+1 \otimes W^{\prime}(M)\right)^{\frac{1}{2}}} \int D X e^{-\operatorname{Tr} V(M)+\operatorname{Tr} W(M) X}= \\
=\frac{\operatorname{det}_{i j} \varphi_{i}^{W}\left(m_{j}\right)}{\prod_{i<j}\left(m_{i}-m_{j}\right)}=\mathcal{T}^{W}(\mathbf{T}), \\
V^{\prime}(M) \equiv W(M),
\end{gathered}
$$

где $m_{i}-$ по-прежнему собственные значения $M$,

$$
\varphi_{i}^{W}(m)=\sqrt{W^{\prime}(\mu)} e^{-V(m)+m W(m)} \int d x x^{i-1} e^{-V(m)+W(m) x},
$$

a $\mathcal{T}^{W}(\mathbf{T})$ - тау-функция ( -редуцированной иерархии КП), удовлетворяющая дополнительным соотношениям, среди которых центральным является струнное уравнение

$$
\begin{aligned}
L_{-1}^{W} \ln Z= & \sum_{n \geqslant 1} \operatorname{Tr}\left(\frac{1}{W^{\prime}(M) M^{n+1}}\right) \frac{\partial \ln Z}{\partial T_{n}}-\frac{\partial \ln Z}{\partial T_{1}}+ \\
& +\frac{1}{2} \sum_{i, j} \frac{1}{W^{\prime}\left(m_{i}\right) W^{\prime}\left(m_{j}\right)} \frac{W^{\prime}\left(m_{i}\right)-W^{\prime}\left(m_{j}\right)}{m_{i}-m_{j}}=0 .
\end{aligned}
$$

Чистая топологическая гравитация отвечает суперпотенциалу

$$
W(X)=X^{2}+t_{0}
$$

Струнное уравнение $L_{-1}^{W} \ln Z=0$ принимает вид

$$
L_{-1}^{\{p\}} \ln Z=\frac{1}{p} \sum_{n \geqslant 1}(n+p) T_{n+p} \frac{\partial}{\partial T_{n}}-\frac{\partial}{\partial T_{1}} \ln Z+\frac{1}{2 p} \sum_{\substack{a+b=p \\ a, b \geqslant 0}} a T_{a} b T_{b}=0
$$

для суперпотенциала $W(X)=X^{p}$, воспроизводя первое $(n=-1)$ уравнение системы (194), если $p=2$. Тот факт, что производяшая функция $\mathcal{T}^{W}$ является тау-функцией иерархии КП, следует немедленно из детерминантного представления (195), которое является хорошо известным детерминантным представлением тау-функции иерархии КП в переменных Мивы [57]. 
Для суперпотенциала $W(X)=X^{p}$ формула (195) представляет собой тау-функцию $p$-редуцированной иерархии КП: случай $p=2$ отвечает иерархии $\mathrm{K}$ д,$p=3$ - иерархии уравнения Буссинеска и т.д. В случае полинома обшего вида мы имеем дело все равно с $p$-редукцией иерархии; чтобы увидеть это, формулу (195) следует переписать в виде [60] (см. также [61, 62])

$$
Z_{\mathrm{GKM}}=\mathcal{T}^{W}=\exp \left\{-\frac{1}{2} \sum_{i, j} A_{i j}(t)\left(t_{i}+T_{i}\right)\left(t_{j}+T_{j}\right)\right\} \mathcal{T}^{(p)}\left(t_{k}+T_{k}\right) \mathcal{T}_{0}(t)
$$

(GKM - обобщенная модель Концевича), где

$$
\begin{gathered}
\frac{\mathcal{T}^{(p)}\left(t_{k}+T_{k}\right)}{\mathcal{T}_{0}(t)}=\frac{\operatorname{det}_{i j} \varphi_{i}^{(p)}\left(\mu_{j}\right)}{\prod_{i<j}\left(\mu_{i}-\mu_{j}\right)}, \\
\varphi_{i}^{(p)}(\mu)=\sqrt{p \mu^{p-1}} e^{-\frac{p}{p+1} \mu^{p+1}} \int d x x^{i-1} e^{-V(x)+\mu^{p} x}, \quad \mu^{p}=W(m)
\end{gathered}
$$

и

$$
\mathcal{T}_{0}(t)=\exp \left\{\frac{1}{2} \sum_{i, j} A_{i j}(t) t_{i} t_{j}\right\}
$$

$\mathrm{a}\left\{t_{k}\right\}$ определены формулой $(164)\left(t_{p+1}=p /(p+1)\right.$ и $t_{l} \equiv 0$ для $\left.l>p+1\right)$, в то время как $A_{i j}(t)=A_{i j}=\operatorname{Res}\left(W^{i}(\lambda) d W_{+}^{j}\right)$. Дополнительный фактор $\mathcal{T}_{0}(t)$ в формуле $(201)$ является квазиклассической уиземовской тау-функцией [60-62], удовлетворяющей уравнениям (ср. с (77))

$$
\begin{gathered}
\frac{\partial \ln \mathcal{T}_{0}(t)}{\partial t_{k}}=t_{-k} \\
\frac{\partial^{2} \ln \mathcal{T}_{0}(t)}{\partial t_{i} \partial t_{j}}=A_{i j}(t)=\operatorname{Res}\left(W^{i}(\lambda) d W_{+}^{j}\right) .
\end{gathered}
$$

Аналогичные утверждения могут быть получены (и были исторически сначала получены) для моделей 2D квантовой ( “физической”) гравитации - непрерывного предела матричных моделей двумерной гравитации [63]. С точки зрения непрерывной теории эти модели представляют собой $(p, q)$-конформную материю (с центральным зарядом $\left.c_{p, q}=1-6(p-q)^{2} /(p q)\right)$, взаимодействующую с лиувиллевской гравитацией с $c_{\mathrm{L}}=$ $26-c_{p, q}=1+6(p+q)^{2} /(p q)$. С этой точки зрения топологические теории, обсуждаемые в п. 4.2 , являются "топологически деформированными" $(p, 1)$-моделями - "чистые” $(p, 1)$-теории отвечают моделям Гинзбурга-Ландау с суперпотенциалами $W(X)=X^{p}$.

Общая схема для $(p, q)$-моделей $2 \mathrm{D}$ квантовой гравитации включает следующие пункты.

A. Условия Вирасоро. Начинать следует с решений $W_{p}$-условий: действия генераторов расширенной алгебры Вирасоро, включаюшей собственно вирасоровские гене- 
раторы

$$
\begin{aligned}
L_{n}= & \frac{1}{p} \sum_{\substack{k>\delta_{n+1,0} \\
k(\bmod p)}} k T_{k} \frac{\partial}{\partial T_{k+p n}}+\frac{1}{2 p} \sum_{\substack{a+b=2 n \\
a, b>0 ; \\
a, b(\bmod p)}} \frac{\partial^{2}}{\partial T_{a} \partial T_{b}}+ \\
& +\delta_{n+1,0} \cdot \frac{1}{2 p} \sum_{a+b=p} a T_{a} b T_{b}+\delta_{n, 0} \cdot \frac{1}{16},
\end{aligned}
$$

обращающего в нуль $p$-редуцированную тау-функцию, или с решения $p$-редуцированной иерархии КП, удовлетворяющего р-струнному уравнению (199).

Б. Критические точки. Согласно [64, 65] производящая функция для данной $(p, q)$-модели может быть получена как решение обобшенных условий Вирасоро (204), если рассматривать тау-функцию как формальный ряд в окрестности следующих “фоновых" значений времен-аргументов:

$$
T_{k} \rightarrow T_{k}+t_{k}=T_{k}+\frac{p}{p+q} \delta_{k, p+q}
$$

где $T_{k}$ - малые параметры формального разложения в ряд. Данная процедура обычно называется выделением $q$-й критической точки в решении $p$-редуцированной иерархии и $W_{p}$-условий. Заметим, что в формуле (199) уже подразумевается сдвиг (205), отвечающий $k=1,-$ именно он приводит к появлению второго члена в правой части (199). Если пользоваться общим правилом (205), то вид этого члена, конечно, будет меняться. В формуле (204) все сдвиги (205) “спрятаны” в переопределение времен T.

Вирасоровские генераторы (204) имеют вид генераторов конформной симметрии в теории одного скалярного поля с антипериодическими граничными условиями. Сушествование вирасоровских условий является универсальным свойством теорий $2 \mathrm{D}$ топологической и квантовой гравитации. Недавно существование вирасоровских условий было обнаружено во взаимодействующих с гравитацией топологических сигма-моделях [66], отвечающих квантовым когомологиям, рассмотренным в п. 4.1.

В. Матричные модели. "Дискретные” условия Вирасоро. Нетривиальные критические точки возникают в непрерывном двойном скейлинговом пределе матричных моделей $2 \mathrm{D}$ квантовой гравитации [64]

$$
Z_{N}=\int \frac{D M_{N \times N}}{\operatorname{Vol} U(N)} e^{-\operatorname{Tr} V(M)},
$$

где интегрирование ведется по эрмитовым $(N \times N)$-матрицам (в общем случае по набору матриц). Для потенциала общего вида $V(M)=\sum t_{k} M^{k}$ матричный интеграл (206) удовлетворяет условиям

$$
\begin{gathered}
L_{n}^{\text {discr }} Z[t]=0, \quad n \geqslant-1 \\
L_{n}^{\text {discr }} \equiv \sum_{k=0}^{\infty} k t_{k} \frac{\partial}{\partial t_{k+n}}+\sum_{a+b=n} \frac{\partial^{2}}{\partial t_{a} \partial t_{b}} \\
\frac{\partial}{\partial t_{0}} Z_{N} \equiv N Z_{N}
\end{gathered}
$$


и эти уравнения в двойном скейлинговом пределе переходят в (204). Производяшая функция (206) $Z_{N}=\mathcal{T}_{N}(t)$ является тау-функцией иерархии полубесконечной цепочки Тоды $[67,68]$, которая в двойном скейлинговом пределе преврашается в тау-функцию иерархии КдФ.

Г. Интегральные формулы и $p q$-дуальность. В отличие от топологической гравитации и топологических струн для точных решений моделей $2 \mathrm{D}$ квантовой гравитации не сушествует явных интегральных представлений (матричный интеграл (206) не является решением непрерывной $2 \mathrm{D}$ гравитации до взятия двойного скейлингова предела). Однако сушествует обобшение интегральных формул (192), (193) и (195) и на этот случай. Разница заключается в том, что теперь вместо интегрального представления для точного решения они приобретают смысл преобразований дуальности между $(p, q)-$ и $(q, p)$-моделями, взаимодействуюшими с $2 \mathrm{D}$ квантовой гравиташией [61]

$$
\begin{aligned}
Z^{(p, q)}(M)= & \sqrt{\operatorname{det} W^{\prime}(M)} \exp \left\{-\operatorname{Tr} \int^{M} Q d W\right\} \int D X \sqrt{\operatorname{det} Q^{\prime}(X)} \times \\
& \times \exp \left\{-\operatorname{Tr} \int^{X} W d Q+\operatorname{Tr} W(M) Q(X)\right\} Z^{(q, p)}(X),
\end{aligned}
$$

где $Q(X)$ - полином степени $q$, дуальный $W(X)$ - полиному степени $p$.

Д. Струнное уравнение как деформация конечнозонного условия. Переписанное для оператора Лакса струнное уравнение приобретает вид [69]

$$
[\mathcal{L}, \mathcal{A}]=1
$$

где $\mathcal{L}=\partial^{p}+\cdots$ - оператор Лакса $p$-редуцированной иерархии КП, символом которого является полином $W(X)$ степени $p$, а $\mathcal{A}=\partial^{q}+\cdots$ - вспомогательный оператор, символ которого - полином $Q(X)$ степени $q$. Легко заметить, что формула (209) представляет собой квантовую деформацию конечнозонного условия (31) [70]. Это говорит о том, что можно пытаться строить решения струнного уравнения исходя из более простых решений интегрируемых систем, удовлетворяюших (31) и задаваемых в обшем случае конструкцией Кричевера.

4.3. Теория Виттена-Зайберга, иерархии Уизема и уравнения ренормгруппы. Обсудив двумерные модельные примеры, обратимся непосредственно к интегрируемым уравнениям уиземовского типа, возникаюшим в теории Виттена-Зайберга. Начнем с разбора некоторых важных примеров явных решений уравнений Уизема.

Рациональные кривые. Простейшие решения систем (66), (68) отвечают простейшим комплексным многообразиям - сферам с отмеченными точками. В случае одной отмеченной точки решения описываются формулой

$$
W=\lambda^{n}+u_{n-2} \lambda^{n-2}+\cdots+u_{0},
$$

определяющей набор функций с полюсами в точке $\lambda=\infty$

$$
\Omega_{i}=W(\lambda, t)_{+}^{\frac{i}{n}}=\lambda^{i}+O\left(\lambda^{i-n}\right)=W^{\frac{i}{n}}+O\left(W^{\frac{i}{n}-1}\right) .
$$


В случае $n=2$ формулы (210), (211) принимают вид

$$
\begin{aligned}
& W=\lambda^{2}+u, \\
& \Omega_{1}=\lambda=\sqrt{W-u}, \\
& \Omega_{3}=\lambda^{3}+\frac{3}{2} u \lambda=(W-u)^{\frac{3}{2}}+\frac{3}{2} u \sqrt{W-u}
\end{aligned}
$$

и т.д., и их можно рассматривать как бездисперсионный предел $n$-редуцированной иерархии КП. Действительно, "нульзонное" или тривиальное решение Кд $\Phi$ ( $n=2$ редукция КП) имеет вид

$$
U=u=\text { const, }
$$

а соответствуюшая функция БА удовлетворяет уравнению

$$
\left(\partial_{T_{1}}^{2}+u\right) \Psi=W \Psi, \quad \partial_{T_{k}} \Psi=\left(\partial_{T_{1}}^{2}+u\right)_{+}^{\frac{k}{2}} \Psi
$$

и имеет вид

$$
\Psi(\lambda, t)=\exp \left\{\sum_{k>0} t_{k} W^{\frac{k}{2}}(\lambda)_{+}\right\} .
$$

Набор функций (211), (212) можно определить в терминах (215) (и естественного обобщения на случай произвольного $n$ ) следующим образом:

$$
\Omega_{k}=\overline{\ln \Psi(\lambda, t)_{t_{k}}}=W^{\frac{k}{n}}(\lambda)_{+} .
$$

Производящий дифференциал (72) и потенциал (71) в этом случае принимают вид

$$
d S=\sum t_{k} d \Omega_{k}, \quad S=\sum t_{k} \Omega_{k}
$$

и решение может быть описано в терминах "периодов" - вычетов в отмеченных точках (cp. c (164))

$$
t_{k}=\left.\frac{1}{k} \operatorname{Res}\right|_{\infty}\left(W^{-\frac{k}{n}} d S\right)
$$

Производные (217) по $t_{k}$ при постоянном $W$ приводят к $(211)$, если $t_{n}=n /(n+1)(\mathrm{cp}$. с (205)!). Например, в случае $n=2$, используя следуюшее из (218) равенство $t_{1}=-u$, имеем

$$
\begin{aligned}
S & =t_{3} \Omega_{3}+t_{2} \Omega_{2}+t_{1} \Omega_{1}=t_{3}\left(\lambda^{3}+\frac{3}{2} u \lambda\right)+t_{2}\left(\lambda^{2}+u\right)+t_{1} \lambda= \\
& =t_{3}\left((W-u)^{\frac{3}{2}}+\frac{3}{2} u \sqrt{W-u}\right)+t_{2} W+t_{1} \sqrt{W-u}
\end{aligned}
$$

и

$$
\begin{aligned}
& \left.\frac{\partial S}{\partial t_{3}}\right|_{W=\mathrm{const}}=(W-u)^{\frac{3}{2}}+\frac{3}{2} u \sqrt{W-u}=\Omega_{3}(W) \\
& \left.\frac{\partial S}{\partial t_{1}}\right|_{W=\mathrm{const}}=\sqrt{W-u}\left(\bmod \left(t_{3}-\frac{2}{3}\right)\right)=\Omega_{1}(W) \quad\left(\bmod \left(t_{3}-\frac{2}{3}\right)\right)
\end{aligned}
$$


Уравнения Уизема (69)

$$
\left.\partial_{t_{3}} \Omega_{1}\right|_{W=\mathrm{const}}=\left.\partial_{t_{1}} \Omega_{3}\right|_{W=\mathrm{const}}
$$

эквивалентны уравнению Хопфа или бездисперсионному уравнению Кд $\Phi$

$$
u_{t_{3}}-\frac{3}{2} u u_{t_{1}}=0
$$

с обшим решением

$$
t_{1}+\frac{3}{2} t_{3} u+P(u)=0, \quad P(u)=\sum t_{2 n+1} u^{n} .
$$

Коэффициенты $t_{2 n+1}$ полинома $P(u)(223)$ при $n>1$ можно рассматривать как параметры общего решения уравнений Уизема (221), (222). Потенциал (219), (220) отвечает случаю, который уже обсуждался в п. 4.2 , когда $t_{2 n+1}=0$ при $n>1$, а $t_{3}=2 / 3$. Это конкретное решение уравнения (223) выделено также тем обстоятельством, что оно является точным решением "полного" уравнения $\mathrm{K} д \Phi$

$$
u=-\frac{2}{3} \frac{t_{1}}{t_{3}}
$$

При этом решение вспомогательной линейной задачи уравнения КдФ

$$
\left(\partial_{t_{1}}^{2}-\frac{2}{3} \frac{t_{1}}{t_{3}}\right) \Psi=\mu^{2} \Psi
$$

имеет вид

$$
\begin{gathered}
\left.\Psi\left(t_{1}, \mu\right)\right|_{t_{3}=\frac{2}{3}}=\operatorname{Ai}\left(t_{1}+\mu^{2}\right), \\
\phi_{i}(\mu) \sim \frac{\partial^{i-1} \Psi(\mu, t)}{\partial t_{1}^{i-1}} \sim \sqrt{2 \mu} e^{-\frac{2}{3} \mu^{3}} \int d x x^{i-1} e^{-\frac{x^{3}}{3}+x \mu^{2}},
\end{gathered}
$$

так что детерминантная формула (201)

$$
\frac{\mathcal{T}(t+T)}{\mathcal{T}(t)}=\frac{\operatorname{det} \phi_{i}\left(\mu_{j}\right)}{\Delta(\mu)}
$$

является тау-функцией “полной” иерархии в переменных Мивы

$$
T_{k}=-\frac{1}{k} \sum_{j} \mu_{j}^{-k} .
$$

Как уже было отмечено в п. 4.2, разложение $\ln \mathcal{T}(T)$ дает корреляторы двумерной топологической гравитации.

Пертурбативная теория Виттена-Зайберга отвечает рациональным кривым с несколькими отмеченными точками $(N$ точек для группы $S U(N))$. Производяший дифференциал (25) в пределе $\Lambda \rightarrow 0$ принимает вид

$$
d S=\lambda d \ln w=\lambda d \ln P_{N}(\lambda)=\sum_{i=1}^{N} \frac{\lambda d \lambda}{\lambda-\phi_{i}}=N d \lambda+\sum_{i=1}^{N} \phi_{i} \frac{d \lambda}{\lambda-\phi_{i}} .
$$


Это частный случай производящих дифференциалов или потенциалов вида

$$
S=x \lambda+\sum_{i=1}^{N} t_{i} \ln \left(\lambda-\phi_{i}\right)+S_{0}, \quad d S=x d \lambda+\sum_{i=1}^{N} t_{i} \frac{d \lambda}{\lambda-\phi_{i}}
$$

который можно рассматривать как вырождение формулы (87) при $t_{i}=\alpha_{i}$ и $t_{n}=0$. Эти дифференциалы возникают как производные

$$
\left.\frac{\partial d S}{\partial t_{k}}\right|_{w=\mathrm{const}}=\left.\frac{\partial \lambda}{\partial t_{k}}\right|_{w=\mathrm{const}} d \ln w=\sum_{i} \frac{d \lambda}{\lambda-\phi_{i}} \frac{\partial \phi_{i}}{\partial t_{k}}
$$

где $d \Omega_{i}=d \lambda /\left(\lambda-\phi_{i}\right)$, если $\partial \phi_{i} / \partial t_{k}=\delta_{i k}$. Следуюшее из (66) уравнение

$$
\partial_{i} \Omega_{j}-\partial_{j} \Omega_{i}+\left\{\Omega_{i}, \Omega_{j}\right\}=\partial_{i} \Omega_{j}-\partial_{j} \Omega_{i}+\frac{\partial \Omega_{i}}{\partial x} \frac{\partial \Omega_{j}}{\partial \lambda}-\frac{\partial \Omega_{j}}{\partial x} \frac{\partial \Omega_{i}}{\partial \lambda}=0
$$

приводит к системе

$$
\partial_{i} \phi_{j}=\partial_{j} \phi_{i}, \quad \partial_{x}\left(\phi_{i}-\phi_{j}\right)=\left(\phi_{i}-\phi_{j}\right) \partial_{i} \phi_{j}
$$

с решением

$$
\phi_{i}=t_{i}, \quad \partial_{x} \phi_{i}=0,
$$

откуда для фиксированного $x=N$ получаем (228).

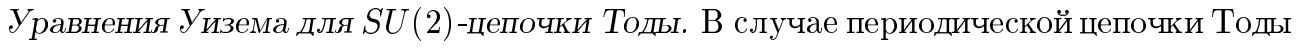
с двумя узлами и спектральной кривой

$$
y^{2}=\left(\lambda^{2}-u\right)^{2}-1
$$

можно ограничиться рассмотрением лишь первых двух дифференциалов $d \Omega_{0}$ и $d \Omega_{1}$ и первых двух времен $t_{0}$ и $t_{1}$. Тогда уравнения Уизема (69) сводятся к единственному уравнению

$$
\frac{\partial d \Omega_{0}}{\partial t_{1}}=\frac{\partial d \Omega_{1}}{\partial t_{0}}
$$

Чтобы написать его явно, следует вспомнить, что:

1) сушествуют два независимых дифференциала

$$
\begin{aligned}
& d \Omega_{0}=d z+\gamma_{0} \frac{d \lambda}{y}=\left(1+\frac{\gamma_{0}}{2 \lambda}\right) d z \\
& d \Omega_{1}=\lambda d z+\gamma_{1} \frac{d \lambda}{y}=\left(\lambda+\frac{\gamma_{1}}{2 \lambda}\right) d z
\end{aligned}
$$

и два соответствуюших уиземовских времени $t_{0}$ и $t_{1}$;

2) коэффициенты $\gamma_{i}(i=0,1)$ определяются, как обычно, обращением в нуль соответствующих А-периодов:

$$
\oint_{A} d \Omega_{i}=0
$$


т.е.

$$
\gamma_{1}=-\frac{a}{\sigma}, \quad \gamma_{0}=-\frac{1}{\sigma}
$$

где

$$
a=\oint_{A} d S=\oint_{A} \lambda d z, \quad \sigma=\oint_{A} \frac{d \lambda}{y}=\frac{\partial a}{\partial u}
$$

3 ) производные по модулям в (235) вычисляются при фиксированных значениях переменной $z$, в то время как переменная $\lambda$ зависит от модулей и эта зависимость определяется уравнением (234), откуда следует

$$
\frac{\partial \lambda}{\partial u}=\frac{1}{2 \lambda}, \quad \frac{\partial a}{\partial u}=\oint_{A} \frac{\partial \lambda}{\partial u} d z=\oint_{A} \frac{d z}{2 \lambda}=\sigma
$$

Используя эти соотношения, из (235) легко получается уравнение на модули

$$
\frac{\partial u}{\partial t_{1}}=a(u) \frac{\partial u}{\partial t_{0}}
$$

Явный вид функции $a(u)$ зависит от выбора циклов. Если $\mathbf{A}$-циклы провести вокруг точек $\lambda=\sqrt{u-2 \Lambda^{2}} \equiv r^{-}$и $\lambda=\sqrt{u+2 \Lambda^{2}} \equiv r^{+}$, то

$$
a=\frac{2}{\pi} r^{+} E(k), \quad \sigma=\frac{1}{\pi r^{+}} K(k),
$$

где $K(k)$ и $E(k)$ - полные эллиптические интегралы первого и второго родов, соответственно, а $k=2 / r^{+}$- эллиптический модуль.

Уравнение (241) имеет общее решение [71]

$$
u=F\left(t_{0}+a(u) t_{1}\right)
$$

где $F(x)$ - произвольная функция. Это решение может быть переписано в виде

$$
\Phi(u)=\left(t_{0}+a(u) t_{1}\right),
$$

где $\Phi(u)$ - обратная функция к $F(x)$.

Обратимся теперь к ренормгрупповой задаче, которая естественным образом формулируется на языке иерархий Уизема. По аналогии с 2D топологическими моделями можно считать [36], что и в 4D логарифм производящей функции некоторых топо-

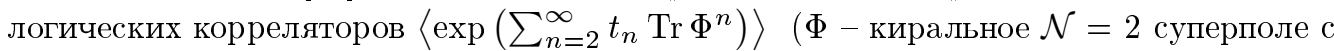
низшей компонентой $\phi$ ) может быть выражен через препотенциал $\mathcal{F}(\boldsymbol{\alpha}, \mathbf{t})$, определяемый семейством спектральных кривых Виттена-Зайберга, параметризованных "плоскими" модулями $\alpha_{i}$. (Собственно препотеншиал Виттена-Зайберга $\mathcal{F}_{\mathrm{SW}}(a)$ возникает в случае замороженных уиземовских времен $t_{n}=\delta_{n, 1}$ и $\alpha_{i}=a_{i}$.) Препотенциал 
$\mathcal{F}(\boldsymbol{\alpha}, \mathbf{t})=\ln \mathcal{T}_{W}(\boldsymbol{\alpha}, \mathbf{t})$ определен как производяшая функция мероморфных 1-форм на спектральных кривых и позволяет непосредственное вычисление величин ${ }^{19)}$

$$
\begin{aligned}
\frac{\partial \mathcal{F}}{\partial t_{n}} & =\frac{\beta}{2 \pi i n} \sum_{m} m t_{m} \mathcal{A}_{m n}=\frac{\beta}{2 \pi i n} t_{1} \mathcal{H}_{n+1}+O\left(t_{2}, t_{3}, \ldots\right), \\
\frac{\partial^{2} \mathcal{F}}{\partial \alpha_{i} \partial t_{n}} & =\frac{\beta}{2 \pi i n} \frac{\partial \mathcal{H}_{n+1}}{\partial a_{i}} \\
\frac{\partial^{2} \mathcal{F}}{\partial t_{m} \partial t_{n}} & =-\frac{\beta}{2 \pi i}\left(\mathcal{A}_{m n}+\frac{\beta}{m n} \frac{\partial \mathcal{H}_{m+1}}{\partial a_{i}} \frac{\partial \mathcal{H}_{n+1}}{\partial a_{j}} \partial_{i j}^{2} \ln \theta_{E}(0 \mid T)\right)
\end{aligned}
$$

и т.д. В формулах (245)-(247) калибровочная группа есть $S U(N)$, параметр $\beta=2 N, \quad m, n=1, \ldots, N-1$, а производные по $t$ вычисляются при постоянных $\alpha_{i}$. Матрица $\mathcal{A}_{m n}$ состоит из определенных однородных комбинаций $h_{k}$, определяемых полиномом $P(\lambda)(13)$ :

$$
\mathcal{A}_{m n}=-\left.\frac{N}{m n} \operatorname{Res}\right|_{\infty}\left(P^{n / N}(\lambda) d P_{+}^{m / N}(\lambda)\right)=\mathcal{A}_{n m},
$$

а также

$$
\mathcal{H}_{n+1} \equiv \mathcal{A}_{n 1}=-\left.\frac{N}{n} \operatorname{Res}\right|_{\infty} P^{n / N}(\lambda) d \lambda=h_{n+1}+O\left(h^{2}\right) .
$$

Анзац Виттена-Зайберга позволяет вычислить производные (245)-(247) при $n=1$, так как после переопределения

$$
h_{k} \rightarrow t_{1}^{k} h_{k}, \quad \mathcal{H}_{k} \rightarrow t_{1}^{k} \mathcal{H}_{k}
$$

можно отождествить $t_{1}$ с $\Lambda=\Lambda_{\mathrm{QCD}}$ - масштабом теории Виттена-Зайберга. Уравнения (245)-(247) для $n=1$ имеют естественньй вид аномалии тензора энергии-импульса

$$
\cdots+\vartheta^{4} \Theta_{\mu}^{\mu}=\beta \operatorname{Tr} \Phi^{2}=\cdots+\vartheta^{4} \beta \operatorname{Tr}\left(\mathbf{F}_{\mu \nu} \mathbf{F}^{\mu \nu}+i \mathbf{F}_{\mu \nu} \widetilde{\mathbf{F}}^{\mu \nu}\right),
$$

так как для любого оператора $\mathcal{O}$ выполняется

$$
\frac{\partial}{\partial \ln \Lambda}\langle\mathcal{O}\rangle=\left\langle\beta \operatorname{Tr} \Phi^{2}, \mathcal{O}\right\rangle
$$

${ }^{19)}$ Операции $\oint$ и Res определены с дополнительными факторами $(2 \pi i)^{-1}$, так что

$$
\left.\operatorname{Res}\right|_{0} \frac{d \xi}{\xi}=-\left.\operatorname{Res}\right|_{\infty} \frac{d \xi}{\xi}=\oint \frac{d \xi}{\xi}=1
$$

Это объясняет появление множителей $2 \pi i$ в тождествах Римана типа (93) и, следовательно, во многих определениях ниже. Соответственно тэта-функции периодичны с периодом $2 \pi i$, а также

$$
\frac{\partial \theta(\mathbf{A} \mid T)}{\partial T_{i j}}=i \pi \partial_{i j}^{2} \theta(\mathbf{A} \mid T)
$$

так как периоды решетки якобиана $A_{i} \equiv \int^{P} d \omega_{i}-2 \pi i(\mathbb{Z}+T \mathbb{Z})$. 
Формула (245) или (252) для $n=1, \mathcal{O}=I$ была получена в работах [72] в виде

$$
\frac{\partial \mathcal{F}_{\mathrm{SW}}}{\partial \ln \Lambda}=\frac{\beta}{2 \pi i}\left(t_{1}^{2} h_{2}\right)
$$

для $\mathcal{O}=h_{m}$, формула $(252)$ (аналог для $n=1$ и любого $m=1, \ldots, N-1$ )

$$
\frac{\partial h_{m}}{\partial \ln \Lambda}=-\beta \frac{\partial h_{2}}{\partial a_{i}} \frac{\partial h_{m}}{\partial a_{j}} \partial_{i j}^{2} \ln \theta_{E}(0 \mid T)
$$

взята из работ $[73,74]$, где она была получена сложным способом как следствие теории Дональдсона - топологического подсектора $\mathcal{N}=2$ суперсимметричной теории Янга-Миллса. В формуле (254) $h_{m}$ можно заменить любой однородной функцией $h$, например $\mathcal{H}_{m}$.

Уравнение (247) можно также переписать в виде

$$
\frac{\partial^{2}}{\partial t_{m} \partial t_{n}}\left(\mathcal{F}(\alpha, t)-\frac{\beta^{2}}{4 \pi i N} \mathcal{F}_{\mathrm{GKM}}(\alpha, t)\right)=-\frac{\beta^{2}}{2 \pi i m n} \frac{\partial \mathcal{H}_{m+1}}{\partial a_{i}} \frac{\partial \mathcal{H}_{n+1}}{\partial a_{j}} \partial_{i j}^{2} \ln \theta_{E}(0 \mid T),
$$

где

$$
\mathcal{F}_{\mathrm{GKM}}(\alpha \mid t) \equiv \frac{1}{2} \sum_{m, n} t_{m} t_{n} \mathcal{A}_{m n}
$$

(ср. с (202)) неявно зависит от модулей $\alpha_{i}$ через коэффициенты полинома $P(\lambda)$.

Наша цель - следуя [36], доказать формулы (245)-(247) для любых $m, n=1, \ldots$ $\ldots, N-1$, а также привести явный вывод уравнения (254), используя теорию римановых поверхностей $[20,21]$. Рассмотрим чистую $S U(N) \mathcal{N}=2$ калибровочную теорию, в которой

$$
P(\lambda)=\lambda^{N}-\sum_{k=2}^{N} u_{k} \lambda^{N-k}=\prod_{i=1}^{N}\left(\lambda-\phi_{i}\right),
$$

a

$$
u_{k}=(-1)^{k+1} \sum_{i_{1}<\cdots<i_{k}} \phi_{i_{1}} \ldots \phi_{i_{k}}
$$

- полиномы Шура от $h_{k}=(1 / k) \sum_{i=1}^{N} \phi_{i}^{k}$ :

$$
\ln \left(\lambda^{-N} P(\lambda)\right)=-\sum_{k} \frac{h_{k}}{\lambda^{k}}
$$

т.е. $u_{0}=1, u_{1}=0, u_{2}=h_{2}, u_{3}=h_{3}, u_{4}=h_{4}-(1 / 2) h_{2}^{2}, \ldots$. Соответствующее семейство спектральных кривых (25) относится к гиперэллиптическим кривым вида

$$
Y^{2}=P^{2}(\lambda)-4 \Lambda^{2 N}, \quad Y=\Lambda^{N}\left(w-\frac{1}{w}\right) .
$$

Среди всех гиперэллиптических кривых отвечаюшие цепочке Тоды (260) выделены, в частности, тем, что на них существует функция $w=(1 / 2) \Lambda^{-N}(P+Y)$ с $N$-кратным 
полюсом и $N$-кратным нулем (так как $\left.w^{-1}=(1 / 2) \Lambda^{-N}(P-Y)\right)$ при $\lambda=\infty_{ \pm}$, т.е. на обоих листах гиперэллиптической кривой.

Из формул (25) и (260) можно получить следуюшее общее соотношение:

$$
\delta P+P^{\prime} \delta \lambda=N P \delta \ln \Lambda+Y \frac{\delta w}{w}
$$

(где $\delta P=-\sum \lambda^{N-k} \delta u_{k}$, а $\left.P^{\prime}=\partial P / \partial \lambda\right)$, которое можно использовать при вычислении различных производных. Например, при постоянных $u_{k}$ и $\Lambda$

$$
\frac{d w}{w}=\frac{P^{\prime} d \lambda}{Y}, \quad d S_{\mathrm{SW}}=\lambda \frac{d w}{w}=\frac{\lambda d P}{Y}
$$

и, следовательно, дифференциалы

$$
\left.\frac{\partial d S_{\mathrm{SW}}}{\partial u_{k}}\right|_{w=\mathrm{const}}=\frac{\lambda^{N-k}}{P^{\prime}} \frac{d w}{w}=\frac{\lambda^{N-k} d \lambda}{Y}=d v_{k}, \quad k=2, \ldots, N,
$$

являются $g=(N-1)$-голоморфными 1-формами на кривой (260) рода $g=N-1$. Их А-периоды равны

$$
\sigma_{i k}=\oint_{A_{i}} d v_{k}=\frac{\partial a_{i}}{\partial u_{k}}
$$

т.е. дифференциалы $d \omega_{i}=\sigma_{i k}^{-1} d v_{k}$ являются каноническими голоморфными 1-дифференшиалами $(37),(38)$. В выражении (263) производные по $u_{k}$ вычисляются при постоянной $w$, при постоянной $\lambda$ получим

$$
\left.\frac{\partial d S_{\mathrm{SW}}}{\partial u_{k}}\right|_{\lambda=\mathrm{const}}=\frac{\lambda^{N-k} d \lambda}{Y}-d\left(\frac{\lambda^{N-k+1}}{Y}\right),
$$

а полные производные будут давать вклад в формулы вычетов. Периоды

$$
a_{i}=\oint_{A_{i}} d S_{\mathrm{SW}}
$$

определяют $a_{i}$ как функции $u_{k}$ (или $h_{k}$ ) и $\Lambda$ или, наоборот, $u_{k}$ как функции $a_{i}$ и $\Lambda$. Из формулы (266) следует, что

$$
\sum_{k}\left(\left.\frac{\partial u_{k}}{\partial \ln \Lambda}\right|_{a_{i}=\text { const }}\right) \oint_{A_{i}} \frac{\partial d S_{\mathrm{SW}}}{\partial u_{k}}+\oint_{A_{i}} \frac{\partial d S_{\mathrm{SW}}}{\partial \ln \Lambda}=0,
$$

так что

$$
\begin{aligned}
\sum_{k} \frac{\partial u_{k}}{\partial \ln \Lambda} \frac{\partial a_{i}}{\partial u_{k}} & =-\oint_{A_{i}} \frac{\partial d S_{\mathrm{SW}}}{\partial \ln \Lambda}=-N \oint_{A_{i}} \frac{P}{P^{\prime}} \frac{d w}{w}=-N \oint_{A_{i}} \frac{P d \lambda}{Y}= \\
& =-N \oint_{A_{i}} \frac{(P+Y) d \lambda}{Y}=-2 N \Lambda^{N} \oint_{A_{i}} \frac{w d \lambda}{Y}= \\
& =-2 N \Lambda^{N} \oint_{A_{i}} w d v_{N} .
\end{aligned}
$$


Более того, поскольку

$$
N P d \lambda=\lambda d P-\sum_{k} k u_{k} \lambda^{N-k} d \lambda,
$$

с учетом (262)-(264) получим из (268)

$$
-\sum_{k} \frac{\partial u_{k}}{\partial \ln \Lambda} \frac{\partial a_{i}}{\partial u_{k}}=a_{i}-\sum_{k} k u_{k} \frac{\partial a_{i}}{\partial u_{k}}
$$

или

$$
\frac{\partial u_{k}}{\partial \ln \Lambda}=k u_{k}-a_{i} \frac{\partial u_{k}}{\partial a_{i}} .
$$

Это следует также из размерных соображений. Чтобы упростить полученные формулы, мы положим $\Lambda=1$, т.е. будем считать, что множители $\Lambda$ убираются переопределением $\lambda, u, h$ и $\mathbf{t}$.

Наша цель теперь - применить уравнения (91) и (92) из п. 2.3 к конкретным спектральным кривым (260). Основная проблема заключается в том, что не существует решений условий (86) с единственным полюсом на спектральных кривых (260) иерархии цепочки Тоды (а не КП или КдФ), где естественно выделены две точки, а не одна. Как уже было замечено, на кривых (260) можно определить функцию $w$ с полюсом и нулем порядка $N$ в этих двух точках $\lambda=\infty_{ \pm}$, где знак \pm обозначает два листа в гиперэллиптической параметризации $(260), w\left(\lambda=\infty_{+}\right)=\infty, w\left(\lambda=\infty_{-}\right)=0$. Соответственно существуют два семейства дифференциалов $d \Omega_{n}: d \Omega_{n}^{+}$с полюсом в точке $\lambda=\infty_{+}$и $d \Omega_{n}^{-}$с полюсом в точке $\lambda=\infty_{-}$.

При этом не сушествует дифференциалов $d \widehat{\Omega}^{ \pm}$, а есть только $d \widehat{\Omega}_{n}=d \widehat{\Omega}_{n}^{+}+d \widehat{\Omega}_{n}^{-}$, т.е. условие (86) приводит к полюсам $d \widehat{\Omega}_{n}$ в обеих отмеченных точках. Более того, коэффициенты перед $w^{n / N}$ в точке $\lambda=\infty_{+}$и $w^{-n / N}$ в точке $\lambda=\infty_{-}$совпадают (так как в случае цепочки Тоды совпадает динамика по положительным и отрицательным временам).

Дифференциалы $d \widehat{\Omega}_{n}$ для семейства (260) имеют вид

$$
d \widehat{\Omega}_{n}=R_{n}(\lambda) \frac{d w}{w}=P_{+}^{n / N}(\lambda) \frac{d w}{w} .
$$

Так как

$$
\begin{aligned}
\left.\delta_{\text {moduli }} d \widehat{\Omega}_{n}\right|_{w=\mathrm{const}} & =\left.\delta R_{n}\right|_{w=\mathrm{const}} \frac{d w}{w}= \\
& =\left(\left.\delta R_{n}\right|_{\lambda=\mathrm{const}}+R_{n}^{\prime} \delta \lambda\right) \frac{d w}{w}=\left(\delta R_{n}-R_{n}^{\prime} \frac{\delta P}{P^{\prime}}\right) \frac{P^{\prime} d \lambda}{y},
\end{aligned}
$$

полиномы $R_{n}(\lambda)$ определяются тем требованием, чтобы выражения $P^{\prime} \delta R_{n}-R_{n}^{\prime} \delta P$ имели степень не более $(N-1)$ - тогда дифференциалы $(273)$ голоморфны. Этим свойством тривиально обладают $R_{n}(\lambda)=P_{+}^{n / N}(\lambda)$, где $\left(\sum_{k=-\infty}^{+\infty} c_{k} \lambda^{k}\right)_{+} \equiv \sum_{k=0}^{+\infty} c_{k} \lambda^{k}$, так как

$$
\begin{aligned}
P^{\prime} \delta R_{n}-R_{n}^{\prime} \delta P & =\frac{n}{N}\left[\left(P^{n / N-1} \delta P\right)_{+} P^{\prime}-\left(P^{n / N-1} P^{\prime}\right)_{+} \delta P\right]= \\
& =-\left[\left(P^{n / N-1} \delta P\right)_{-} P^{\prime}-\left(P^{n / N-1} P^{\prime}\right)_{-} \delta P\right]=\mathcal{O}\left(\lambda^{N-2}\right)
\end{aligned}
$$


Например,

$$
\begin{aligned}
& R_{1}=\lambda, \quad R_{2}=\lambda^{2}-\frac{2}{N} u_{2}, \quad R_{3}=\lambda^{3}-\frac{3}{N} u_{2} \lambda-\frac{3}{N} u_{3}, \\
& R_{4}=\lambda^{4}-\frac{4}{N} u_{2} \lambda^{2}-\frac{4}{N} u_{3} \lambda-\left(\frac{4}{N} u_{4}+\frac{2(N-4)}{N^{2}} u_{2}^{2}\right)
\end{aligned}
$$

и т.д. Эти дифференциалы удовлетворяют условию (86), если производные по модулям вычисляются при постоянной $w$ (а не $\lambda$ !). Таким образом, мы можем применять формализм п. 2.3, выбрав координатой $\xi=w^{\mp 1 / N}$.

Производящим дифференциалом Виттена-Зайберга является $d S_{\mathrm{SW}}=d \widehat{\Omega}_{2}$, т.е.

$$
\left.d S\right|_{t_{n}=\delta_{n, 1}}=d S_{\mathrm{SW}},\left.\quad \alpha_{i}\right|_{t_{n}=\delta_{n, 1}}=a_{i},\left.\quad \alpha_{i}^{D}\right|_{t_{n}=\delta_{n, 1}}=a_{i}^{D} .
$$

Таким образом, данные, необходимые для определения препотенциала, зафиксированы следующим образом: отмечены точки $\lambda=\infty_{ \pm}$, соответствующими координатами в этих точках являются $\xi \equiv w^{-1 / N} \sim \lambda^{-1}$ при $\lambda=\infty_{+}$и $\xi \equiv w^{+1 / N} \sim \lambda^{-1}$ при $\lambda=\infty_{-}$. Параметризация с помошью $w$ не позволяет непосредственно пользоваться преимушествами гиперэллиптической координаты $\lambda$, поэтому для промежуточных вычислений мы определим также $d \widetilde{\Omega}$ - дифференциалы, определенные, в отличие от $d \Omega$, формулой (80) при $\xi=\lambda^{-1}$. Вблизи точек $\lambda= \pm \infty$ параметр

$$
w^{ \pm 1}=\frac{1}{2}(P \pm Y)=P(\lambda)\left(1+O\left(P^{-2}\right)\right)=P(\lambda)\left(1+O\left(\lambda^{-2 N}\right)\right) .
$$

Поэтому в вычислениях с $w^{ \pm n / N}$ при $n<2 N$ можно заменить $w^{ \pm 1 / N}$ на $P(\lambda)^{1 / N}$.

A. Первые производные. После сделанных комментариев для $n<2 N$ можно написать

$$
\begin{aligned}
\frac{\partial \mathcal{F}}{\partial t_{n}} & =\frac{N}{2 \pi i n}\left(\left.\operatorname{Res}\right|_{\infty_{+}} w^{n / N} d S+\left.\operatorname{Res}\right|_{\infty_{-}} w^{-n / N} d S\right)= \\
& =\frac{N}{2 \pi i n}\left(\left.\operatorname{Res}\right|_{\infty_{+}} w^{n / N}+\left.\operatorname{Res}\right|_{\infty_{-}} w^{-n / N}\right)\left(\sum_{m} t_{m} P_{+}^{m / N}(\lambda)\right) \frac{d w}{w}= \\
& =\left.\frac{N^{2}}{i \pi n^{2}} \sum_{m} t_{m} \operatorname{Res}\right|_{\infty}\left(P_{+}^{m / N}(\lambda) d P^{n / N}(\lambda)\right)= \\
& =-\left.\frac{N^{2}}{i \pi n^{2}} \sum_{m} t_{m} \operatorname{Res}\right|_{\infty}\left(P^{n / N}(\lambda) d P_{+}^{m / N}(\lambda)\right) .
\end{aligned}
$$

Подставив (277) в (94), можно сравнить полученный результат с препотенциалом (202) [60-62],

$$
\mathcal{F}_{\mathrm{GKM}}^{(N)}(t)=\left.\sum_{m, n} \frac{t_{m} t_{n}}{2 m n} \operatorname{Res}\right|_{\infty}\left(P^{n / N}(\lambda) d P_{+}^{m / N}(\lambda)\right) .
$$

В случае модели Виттена-Зайберга появляется новый трансцендентный вклад $(1 / 2) a_{i} a_{i}^{D}$, возникаюший благодаря нетривиальной спектральной кривой. Зависимость 
полинома $P(\lambda)$, т.е. коэффициентов $u_{k}$, от $t_{m}$ является, безусловно, также трансцендентной - определяемой уравнениями Уизема (99). В случае обобщенной модели Концевича уравнения Уизема решаются алгебраически:

$$
t_{n}^{\mathrm{GKM}}=-\left.\frac{N}{N-n} \operatorname{Res}\right|_{\infty} P^{1-n / N}(\lambda) d \lambda .
$$

Эти величины были впервые введены в [49] для топологических теорий Гинзбурга-Ландау (см. (164)), где $P(\lambda)=W^{\prime}(\lambda)$ - производная суперпотенциала.

Вычисленная при $t_{n}=\delta_{n, 1}$ формула (277) принимает вид

$$
\frac{\partial \mathcal{F}}{\partial t_{n}}=-\left.\frac{N^{2}}{i \pi n^{2}} \operatorname{Res}\right|_{\infty} P^{n / N}(\lambda) d \lambda=\frac{N}{i \pi n} \mathcal{H}_{n+1}
$$

где

$$
\begin{aligned}
\mathcal{H}_{n+1} & \equiv-\left.\frac{N}{n} \operatorname{Res}\right|_{\infty} P^{n / N}(\lambda) d \lambda= \\
& =\sum_{k \geqslant 1} \frac{(-1)^{k-1}}{k !}\left(\frac{n}{N}\right)^{k-1} \sum_{i_{1}+\cdots+i_{k}=n+1} h_{i_{1}} \ldots h_{i_{k}}= \\
& =h_{n+1}-\frac{n}{2 N} \sum_{i+j=n+1} h_{i} h_{j}+O\left(h^{3}\right) .
\end{aligned}
$$

Заметим, что полученные величины как алгебраические комбинации $h_{k}$ не отличаются от (279), но $h_{k}$ безусловно разные функции от $t$ в обобшенных моделях Концевича и теории Виттена-Зайберга.

Б. Смешанные производные. Для смешанных производных получаем

$$
\begin{aligned}
\frac{\partial^{2} \mathcal{F}}{\partial \alpha_{i} \partial t_{n}} & =\oint_{B_{i}} d \Omega_{n}=\left.\frac{1}{2 \pi i n} \operatorname{Res}\right|_{0} \xi^{-n} d \omega_{i}= \\
& =\frac{N}{2 \pi i n}\left(\left.\operatorname{Res}\right|_{\infty_{+}} w^{n / N} d \omega_{i}+\left.\operatorname{Res}\right|_{\infty_{-}} w^{n / N} d \omega_{i}\right)= \\
& =\left.\frac{N}{i \pi n} \operatorname{Res}\right|_{\infty} P^{n / N}(\lambda) d \omega_{i} .
\end{aligned}
$$

Введем специальное обозначение для коэффициентов разложения $P^{n / N}(\lambda)$ :

$$
P^{n / N}(\lambda)=\sum_{k=-\infty}^{n} p_{n k}^{(N)} \lambda^{k},
$$

тогда

$$
\left.\operatorname{Res}\right|_{\infty} P^{n / N}(\lambda) d \omega_{i}=\left.\sum_{k=-\infty}^{n} p_{n k}^{(N)} \operatorname{Res}\right|_{\infty} \lambda^{k} d \omega_{i}
$$


Каноническими дифференциалами являются

$$
\begin{aligned}
d \omega_{j}(\lambda) & =\sigma_{j k}^{-1} d v_{k}(\lambda)=\sigma_{j k}^{-1} \frac{\lambda^{N-k} d \lambda}{Y(\lambda)}= \\
& =\sigma_{j k}^{-1} \frac{\lambda^{N-k} d \lambda}{P(\lambda)}\left(1+O\left(\lambda^{-2 N}\right)\right)= \\
& =-\sigma_{j k}^{-1} \frac{\partial \ln P(\lambda)}{\partial u_{k}} d \lambda\left(1+O\left(\lambda^{-2 N}\right)\right)
\end{aligned}
$$

Из формулы $(259)$ и $\sigma_{j k}^{-1}=\partial u_{k} / \partial a_{j}$ получаем

$$
d \omega_{j}(\lambda)\left(1+O\left(\lambda^{-2 N}\right)\right)=\sum_{n \geqslant 2} \sigma_{j k}^{-1} \frac{\partial h_{n}}{\partial u_{k}} \frac{d \lambda}{\lambda^{n}}=\sum_{n \geqslant 1} \frac{\partial h_{n+1}}{\partial a_{i}} \frac{d \lambda}{\lambda^{n+1}}
$$

так что для $k<2 N$

$$
\left.\operatorname{Res}\right|_{\infty} \lambda^{k} d \omega_{i}=\frac{\partial h_{k+1}}{\partial a_{i}}
$$

Заметим, что вычеты равны нулю для $k<1$, поэтому из (284) для $n<2 N$ следует, что

$$
\left.\operatorname{Res}\right|_{\infty} w^{n / N} d \omega_{i}=\left.\operatorname{Res}\right|_{\infty} P^{n / N}(\lambda) d \omega_{i}=\sum_{k=-\infty}^{n} p_{n k}^{(N)} \frac{\partial h_{k+1}}{\partial a_{i}}=\sum_{k=1}^{n} p_{n k}^{(N)} \frac{\partial h_{k+1}}{\partial a_{i}}
$$

Более того,

$$
\begin{aligned}
\sum_{k=-\infty}^{n} p_{n k}^{(N)} \delta h_{k+1} & =\sum_{k=1}^{\infty} \oint_{\infty} \frac{d \lambda}{\lambda^{k+1}} P^{n / N}(\lambda) \delta h_{k+1}= \\
& =-\oint_{\infty} d \lambda P^{n / N}(\lambda) \delta \ln P(\lambda)= \\
& =-\frac{N}{n} \oint_{\infty} \delta P^{n / N}(\lambda) d \lambda=\delta \mathcal{H}_{n+1}
\end{aligned}
$$

и, наконец,

$$
\left.\operatorname{Res}\right|_{\infty} w^{n / N} d \omega_{i}=\left.\operatorname{Res}\right|_{\infty} P^{n / N}(\lambda) d \omega_{i}=\frac{\partial \mathcal{H}_{n+1}}{\partial a_{i}}
$$

Следовательно

$$
\frac{\partial^{2} \mathcal{F}}{\partial \alpha_{i} \partial t_{n}}=\left.\frac{N}{i \pi n} \operatorname{Res}\right|_{\infty} P^{n / N}(\lambda) d \omega_{i}=\frac{N}{i \pi n} \frac{\partial \mathcal{H}_{n+1}}{\partial a_{i}}
$$


B. Вторые производные по $t$. Выражение (277) не применимо непосредственно к вычислению вторых производных $\mathcal{F}$, так как $P(\lambda)$ зависит от $t$ через коэффициенты $u_{k}$, где $u(t)$ - решения трансцендентных уравнений (99). Поэтому легче использовать общие формулы (92):

$$
\begin{aligned}
\frac{\partial^{2} \mathcal{F}}{\partial t_{m} \partial t_{n}} & =\left.\frac{1}{2 \pi i n} \operatorname{Res}\right|_{0} \xi^{-n} d \Omega_{n}= \\
& =\frac{N}{2 \pi i n}\left(\left.\operatorname{Res}\right|_{\infty_{+}} w^{n / N} d \Omega_{m}+\left.\operatorname{Res}\right|_{\infty_{-}} w^{-n / N} d \Omega_{m}\right)
\end{aligned}
$$

Легче вычислять вычеты с дифференциалами $d \widetilde{\Omega}$ вместо $d \Omega$. Таким образом, нам нужно выражение для $d \Omega_{n}^{ \pm}$через $d \widetilde{\Omega}_{n}^{ \pm}$, которое легко получить из асимптотики вблизи точки $\lambda=\infty$ :

$$
\begin{aligned}
d \Omega_{n}^{ \pm} & = \pm\left(w^{ \pm n / N}+O(1)\right) \frac{d w}{w}=\frac{N}{n} d w^{ \pm n / N}+\cdots=\frac{N}{n} d P^{n / N}+\cdots= \\
& =\frac{N}{n} \sum_{k=1}^{n} k p_{n k}^{(N)} \lambda^{k-1} d \lambda+\cdots=\frac{N}{n} \sum_{k=1}^{n} k p_{n k}^{(N)} d \widetilde{\Omega}_{k}^{ \pm}
\end{aligned}
$$

Здесь многоточием обозначены несингулярные члены, которые определяются сингулярными. Поэтому чтобы установить справедливость формулы (293), достаточно сравнить лишь сингулярные члены $d \Omega_{n}$ и $d \widetilde{\Omega}_{n}$ (так как оба набора отвечают полному базису дифференциалов с нулевыми $\mathbf{A}$-периодами и голоморфными везде, кроме точек $\pm \infty)$.

Подставляя (293) и (283) в (292), для $m, n<2 N$ получим

$$
\frac{\partial^{2} \mathcal{F}}{\partial t_{m} \partial t_{n}}=-\left.\frac{N^{2}}{i \pi m n} \sum_{l=1}^{m} l p_{m l}^{(N)} \operatorname{Res}\right|_{\infty} w^{n / N} d \widetilde{\Omega}_{l}, \quad d \widetilde{\Omega}_{l}=d \widetilde{\Omega}_{l}^{+}+d \widetilde{\Omega}_{l}^{-}
$$

Г. Заключительный шаг. Наконец, можно завершить вычисление (294) для $m, n=$ $1, \ldots, N-1$ :

$$
\begin{aligned}
\frac{\partial^{2} \mathcal{F}}{\partial t_{m} \partial t_{n}} & =\left.\frac{N^{2}}{i \pi m n} \sum_{l=1}^{m} l p_{m l}^{(N)} \operatorname{Res}\right|_{\infty} w^{n / N} d \widetilde{\Omega}_{l}^{+}= \\
& =\left.\frac{N^{2}}{i \pi m n} \sum_{l=1}^{m} l p_{m l}^{(N)} \operatorname{Res}\right|_{\infty} w^{n / N}\left(\lambda^{l-1} d \lambda-2 \rho_{l}^{i} d \omega_{i}(\lambda)\right)
\end{aligned}
$$

Первый член в правой части можно переписать в виде

$$
\begin{aligned}
& \left.\sum_{l=1}^{m} l p_{m l}^{(N)} \operatorname{Res}\right|_{\infty} w^{n / N} \lambda^{l-1} d \lambda=\left.\operatorname{Res}\right|_{\infty}\left(w^{n / N} d P_{+}^{m / N}(\lambda)\right)= \\
& \quad=\left.\operatorname{Res}\right|_{\infty}\left(P^{n / N}(\lambda) d P_{+}^{m / N}(\lambda)\right),
\end{aligned}
$$


второй член может быть вычислен с помощью (290), (289):

$$
-\left.2\left(\sum_{l=1}^{m} l p_{m l}^{(N)} \rho_{l}^{i}\right) \operatorname{Res}\right|_{\infty} w^{n / N} d \omega_{i}(\lambda)=-2 \frac{\partial \mathcal{H}_{m+1}}{\partial a_{j}} \partial_{i j}^{2} \ln \theta_{E}(0 \mid T) \frac{\partial \mathcal{H}_{n+1}}{\partial a_{i}}
$$

Сложив выражения (296) и (297), получим

$$
\begin{aligned}
\frac{\partial^{2} \mathcal{F}}{\partial t_{m} \partial t_{n}}= & \frac{N^{2}}{i \pi m n}\left(\left.\operatorname{Res}\right|_{\infty}\left(P^{n / N}(\lambda) d P_{+}^{m / N}(\lambda)\right)-\right. \\
& \left.-2 \frac{\partial \mathcal{H}_{m+1}}{\partial a_{i}} \frac{\partial \mathcal{H}_{n+1}}{\partial a_{j}} \partial_{i j}^{2} \ln \theta_{E}(0 \mid T)\right)
\end{aligned}
$$

Остальные вторые производные образуют матрицу периодов спектральной кривой (260):

$$
\frac{\partial^{2} \mathcal{F}}{\partial \alpha_{i} \partial \alpha_{j}}=\oint_{B_{i}} d \omega_{j}=T_{i j}
$$

Наконец, обратимся к непосредственному доказательству уравнения (254). С точки зрения общего подхода выражение (254) является частным случаем формулы (247), имеющим смысл в рамках анзаца Виттена-Зайберга без какого-то расширения на всю иерархию Уизема. Поэтому этот случай заслуживает специального рассмотрения, не опираюшегося на иерархии Уизема.

Выведем соотношение (254) из (268). Для этого нужно знать А-периоды дифференциала $(P+Y) / Y d \lambda=2 w d \lambda / Y$. Согласно формулам разложения квадрата ядра Сегё (подробнее см. работу [36] и приведенную в ней библиографию)

$$
\Psi_{E}^{2}(\lambda, \mu)=\frac{P_{N}(\lambda) P_{N}(\mu)-4 \Lambda^{2 N}+Y(\lambda) Y(\mu)}{2 Y(\lambda) Y(\mu)} \frac{d \lambda d \mu}{(\lambda-\mu)^{2}}
$$

около $\mu=\infty_{ \pm}$

$$
\Psi_{E}^{2}(\lambda, \mu)=\sum_{n \geqslant 1} \widehat{\Psi}_{E}^{2}(\lambda) \frac{n \lambda^{n-1} d \mu}{\mu^{n+1}}\left(1+O\left(P_{N}^{-1}(\mu)\right)\right)
$$

где

$$
\widehat{\Psi}_{E}^{2}(\lambda) \equiv \frac{P_{N}(\lambda) \pm Y(\lambda)}{2 Y(\lambda)} d \lambda= \begin{cases}\left(1+O\left(\lambda^{-2 N}\right)\right) d \lambda & \text { около } \infty_{ \pm}, \\ O\left(\lambda^{-2 N}\right) d \lambda & \text { около } \infty_{\mp},\end{cases}
$$

этот дифференциал совпадает с удвоенным квадратом ядра Сегё $2 \Psi_{E}^{2}(\lambda, \infty)$ для конкретной четной тэта-характеристики $E$ и в точке $\mu=\infty$.

А-периоды $\Psi^{2}$ легко вычисляются с помошью [20]

$$
\Psi_{e}\left(P, P^{\prime}\right) \Psi_{-e}\left(P, P^{\prime}\right)=W\left(P, P^{\prime}\right)+d \omega_{i}(P) d \omega_{j}\left(P^{\prime}\right) \frac{\partial^{2}}{\partial A_{i} \partial A_{j}} \ln \theta_{e}(0 \mid T),
$$


поскольку А-периоды $W\left(P, P^{\prime}\right)$ равны нулю (см. (83)). Из (268) и (303) получаем

$$
\begin{aligned}
-\frac{\partial u_{k}}{\partial \ln \Lambda} \frac{\partial a_{i}}{\partial u_{k}} & =2 N \Lambda^{N} \oint_{A_{i}} \frac{w d \lambda}{Y}=N \oint_{A_{i}} \frac{P+Y}{Y} d \lambda= \\
& =2 N \oint_{A_{i}} \widehat{\Psi}_{E}^{2}(\lambda)=2 N \frac{\partial h_{2}}{\partial a_{j}} \partial_{i j}^{2} \ln \theta_{E}(0 \mid T) .
\end{aligned}
$$

Это доказьвает соотношение (254):

$$
\frac{\partial u_{k}}{\partial \ln \Lambda}=-2 N \frac{\partial u_{k}}{\partial a_{i}} \frac{\partial u_{2}}{\partial a_{j}} \partial_{i j}^{2} \ln \theta_{E}(0 \mid T),
$$

здесь можно заменить $u_{k}$ в обеих частях равенства на любую функцию от $u_{k}$ (но не других аргументов!), например на $h_{k}$ или $\mathcal{H}_{k+1}$.

В заключение сделаем несколько замечаний.

1. О производных по $\ln \Lambda$ и $\ln t_{1}$. Согласно соотношению (261) можно записать

$$
P^{\prime} \delta \lambda-\sum_{k} \lambda^{N-k} \delta u_{k}=N P \delta \ln \Lambda
$$

откуда следует, что

$$
\delta a_{i}=\oint_{A_{i}} \delta \lambda \frac{d w}{w}=\sum_{k} \delta u_{k} \oint_{A_{i}} \frac{\lambda^{N-k}}{P^{\prime}} \frac{d w}{w}+N \delta \ln \Lambda \oint_{A_{i}} \frac{P}{P^{\prime}} \frac{d w}{w}
$$

и

$$
\left.\sum_{k} \oint_{A_{i}} d v_{k} \frac{\partial u_{k}}{\partial \ln \Lambda}\right|_{a=\mathrm{const}}=-N \oint_{A_{i}} \frac{P}{P^{\prime}} \frac{d w}{w}=-N \oint_{A_{i}} \frac{P d \lambda}{Y} .
$$

С другой стороны, для $\alpha_{i}=t_{1} a_{i}+O\left(t_{2}, t_{3}, \ldots\right)$,

$$
\delta \alpha_{i}=\alpha_{i} \delta \ln t_{1}+t_{1} \oint_{A_{i}} \delta \lambda \frac{d w}{w}+O\left(t_{2}, t_{3}, \ldots\right)
$$

и постоянных $\Lambda$ и $t_{n}=\delta_{n, 1}$ выполняется

$$
\left.\sum_{k} \oint_{A_{i}} d v_{k} \frac{\partial u_{k}}{\partial \ln t_{1}}\right|_{\alpha=\mathrm{const}}=-\frac{\alpha_{i}}{t_{1}}=-\oint_{A_{i}} \frac{\lambda d P}{Y} .
$$

Поскольку

$$
\lambda d P=N P d \lambda+\sum_{k} k u_{k} \lambda^{N-k} d \lambda
$$

из (308) и (310) следует, что

$$
\left.\frac{\partial u_{k}}{\partial \ln t_{1}}\right|_{\alpha=\text { const }}=\left.\frac{\partial u_{k}}{\partial \ln \Lambda}\right|_{a=\text { const }}-k u_{k}=-a_{i} \frac{\partial u_{k}}{\partial a_{i}},
$$

или в точности соотношение (271). Соотношение (311) безусловно верно для любой однородной алгебраической комбинации $u_{k}$, в частности для $h_{k}$ и $\mathcal{H}_{k}$. 
2. Проверка непротиворечивости. Чтобы проверить согласованность (247) с (254), используем соотношение (245), откуда следует, что

$$
\frac{\beta}{2 \pi i} \mathcal{H}_{k+1}=\frac{k}{t_{1}} \frac{\partial \mathcal{F}}{\partial t_{k}}
$$

и продифференцируем полученное выражение по $t_{1}$. Выражая левую часть с помощью (311) через производную $\mathcal{H}_{k+1}$ по $\ln \Lambda$ и используя (254), найдем

$$
\begin{aligned}
\frac{\beta}{2 \pi i} \frac{\partial \mathcal{H}_{k+1}}{\partial \ln t_{1}} & =\frac{\beta}{2 \pi i}\left(\frac{\partial \mathcal{H}_{k+1}}{\partial \ln \Lambda}-(k+1) \mathcal{H}_{k+1}\right)= \\
& =-\frac{\beta^{2}}{2 \pi i} \frac{\partial \mathcal{H}_{k+1}}{\partial a_{i}} \rho_{1}^{i}-(k+1) \frac{\beta}{2 \pi i} \mathcal{H}_{k+1} .
\end{aligned}
$$

Выразим правую часть (313) через вторые производные препотенциала и применим (247):

$$
\begin{aligned}
\frac{\beta}{2 \pi i} \frac{\partial \mathcal{H}_{k+1}}{\partial \ln t_{1}} & =k \frac{\partial^{2} \mathcal{F}}{\partial t_{1} \partial t_{k}}-\frac{\beta}{2 \pi i} \mathcal{H}_{k+1}= \\
& =\frac{\beta^{2}}{2 \pi i}\left(\left.\frac{1}{2} \operatorname{Res}\right|_{\infty}\left(P^{k / N}(\lambda) d P_{+}^{1 / N}(\lambda)\right)-\frac{\partial \mathcal{H}_{k+1}}{\partial a_{i}} \rho_{1}^{i}\right)-\frac{\beta}{2 \pi i} \mathcal{H}_{k+1}
\end{aligned}
$$

Поскольку $P_{+}^{1 / N}(\lambda)=\lambda$, можно воспользоваться формулой $(281)$ :

$$
\left.\beta \operatorname{Res}\right|_{\infty}\left(P^{k / N}(\lambda) d P_{+}^{1 / N}(\lambda)\right)=-2 k \mathcal{H}_{k+1}
$$

(так как $\beta=2 N$ ). Мы видим, что правые части (313) и (314) идентичны. Это доказывает непротиворечивость (247) и (254) или, другими словами, представляет собой прямой вывод соотношения (254) из (245) и (247). Уравнение (254) является частным случаем (247) при $t_{2}=t_{3}=\cdots=0$.

Обобщение соотношения (271) на общий случай ненулевых времен может быть получено подстановкой формул (245)-(247) в соотношение однородности (94), которое подразумевает, что

$$
\frac{\partial \mathcal{F}}{\partial t_{n}}=\sum_{i} \alpha_{i} \frac{\partial^{2} \mathcal{F}}{\partial \alpha_{i} \partial t_{n}}+\sum_{m} t_{m} \frac{\partial^{2} \mathcal{F}}{\partial t_{m} \partial t_{n}} .
$$

Подстановка (245)-(247) приводит для $n=1, \ldots, N-1$ и $t_{m \geqslant N}=0 \mathrm{k}$

$$
\alpha_{i} \frac{\partial \mathcal{H}_{n+1}}{\partial a_{i}}=\sum_{m} t_{m}\left((m+n) \mathcal{A}_{m n}+\frac{\beta}{m} \frac{\partial \mathcal{H}_{m+1}}{\partial a_{i}} \frac{\partial \mathcal{H}_{n+1}}{\partial a_{j}} \partial_{i j}^{2} \ln \theta_{E}(0 \mid T)\right)
$$

При $t_{m}=\delta_{m, 1}$ с учетом (254) формула (317) преврашается в $(271)$. 


\section{5. ЗАКЛЮЧЕНИЕ}

В этой работе мы постарались продемонстрировать, как общие постулаты непертурбативной теории струн дают возможность получить точную информацию о вакуумной структуре низкоэнергетических эффективных квантовых теорий поля. Мы также показали, что структура пространств модулей струнных компактификаций естественным образом приводит к возникновению интегрируемых структур, которые, как известно, возникают при описании точных решений топологических струнных моделей и эффективных теорий Виттена-Зайберга.

Благодарности. Я благодарен Г. Брадену, А. Вайнштейну, А. Герасимову, А. Горскому, И. Кричеверу, А. Лосеву, А. Миронову, А. Морозову, А. Рослому, В. Фоку, А. Ханани, С. Харчеву, С. Черкису, Дж. Шварцу и А. Юнгу за обсуждение различных проблем, рассмотренных в данной работе, и А. Погребкову за предложение написать настоящую статью.

Работа частично поддержана грантами РФФИ № 99-02-16122 и INTAS № 97-0103.

\section{Список литературы}

[1] М. Грин, Дж. Швари, Э. Виттен. Теория суперструн. Т. 1, 2. М.: Мир, 1990.

[2] R. Dijkgraaf, E. Verlinde, H. Verlinde. Commun. Math. Phys. 1988. V. 115. P. 649; P. Ginsparg. Nucl. Phys. B. 1988. V. 295 [FS21]. P. 153.

[3] J. Polchinski. Phys. Rev. Lett. 1995. V. 75. P. 4724; hep-th/9510017; Progr. Theor. Phys. Suppl. 1996. V. 123. P. 9; hep-th/9511157; Rev. Mod. Phys. 1996. V. 68. P. 1245; hep-th/9607050; TASI lectures on D-branes. hep-th/9611050.

[4] P. Townsend. Phys. Lett. B. 1995. V. 350. P. 184; hep-th/9501068; E. Witten. Nucl. Phys. B. 1995. V. 443. P. 85; hep-th/9503124; P. Townsend. Four lectures on M-theory. hep-th/9612121; J. Schwarz. Lectures on superstring and M-theory dualities. hep-th/9607201; C. Vafa. Lectures on strings and dualities. hep-th/9702201.

[5] E. Witten. Vector bundles and F-theory. hep-th/9703166.

[6] E. Witten. Nucl. Phys. B. 1996. V. 460. P. 335; hep-th/9510135.

[7] D.-E. Diaconescu. D-branes, monopoles, and Nahm equations. hep-th/9608163.

[8] A. Hanany, E. Witten. Nucl. Phys. B. 1997. V. 492. P. 152; hep-th/9611230.

[9] R. Donagi, E. Witten. Nucl. Phys. B. 1996. V. 460. P. 299; hep-th/9510101; E. Martinec. Phys. Lett. B. 1996. V. 367. P. 91; hep-th/9510204; A. Gorsky, A. Marshakov. Phys. Lett. B. 1996. V. 375. P. 127; hep-th/9510224; H. Itoyama, A. Morozov. Nucl. Phys. B. 1996. V. 477. P. 855; hep-th/9511125; E. D'Hoker, D. Phong. Calogero-Moser systems in $S U(N)$ Seiberg-Witten theory. hep-th/9709053; Spectral curves for super-Yang-Mills with adjoint hypermultiplet for general Lie algebras. hep-th/9804126.

[10] N. Seiberg, E. Witten. Nucl. Phys. B. 1994. V. 426. P. 19; hep-th/9407087.

[11] N. Seiberg, E. Witten. Nucl. Phys. B. 1994. V. 431. P. 484; hep-th/9408099.

[12] A. Klemm, W. Lerche, S. Theisen, S. Yankielowicz. Phys. Lett. B. 1995. V. 344. P. 169; hep-th/9411048; P. Argyres, A. Faraggi. Phys. Rev. Lett. 1995. V. 73. P. 3931; hep-th/9411057.

[13] K. Wilson, J. Kogut. Phys. Rep. C. 1974. V. 12. P. 75.

[14] M. Shifman, A. Vainshtein. Nucl. Phys. B. 1986. V. 277. P. 456.

[15] N. Seiberg. The power of holomorphy - exact results in 4D SUSY field theory. hep-th/9408013.

[16] A. Gorsky, I. Krichever, A. Marshakov, A. Mironov, A. Morozov. Phys. Lett. B. 1995. V. 355. P. 466; hep-th/9505035. 
[17] В. Захаров, С. Манаков, С. Новиков, Л. Питаевский. Теория солитонов: Методобратной задачи. М.: Наука, 1980.

[18] Б. Дубровин. УМН. 1981. Т. 36. № 2. С. 12.

[19] И. Кричевер. Функц. анализ и его прилож. 1977. Т. 11. С. 15; УМН. 1977. Т. 32. С. 186.

[20] J. Fay. Theta-functions on Riemann surfaces. Lect. Notes in Math. V.352. N. Y.: Springer, 1973.

[21] D. Mumford. Tata Lectures on Theta. V. I, II. Boston-Basel-Stuttgart: Birkhäuser, 1983, 1984.

[22] Б. Дубровин, И. Кричевер, С. Новиков. Интегрируемые системы. І.В сб.: Итоги науки и техники. Сер.: Совр. пробл. матем. Динамические системы - IV. Ред. Р. В. Гамкрелидзе. М.: ВИНИТИ, 1985. С. 179.

[23] N. Hitchin. Duke Math. J. 1987. V. 54. P. 91.

[24] A. Gorsky, N. Nekrasov. Elliptic Calogero-Moser system from two dimensional current algebra. hep-th/9401021.

[25] N. Nekrasov. Holomorphic bundles and many-body systems. hep-th/9503157.

[26] A. Levin, M. Olshanetsky. Painlevé-Calogero correspondence. alg-geom/9706010; Classical limit of the Knizhnik-Zamolodchikov-Bernard equations as hierarchy of isomonodromic deformations. Free fields approach. hep-th/9709207.

[27] A. Marshakov. Seiberg-Witten theory, integrable systems and D-branes. In: New Developments in Quantum Field Theory. NATO ASI Series B: Physics. V. 366. Eds. P.H. Damgaard, J. Jurkiewicz. N. Y.: Plenum Press, 1999. P. 279; hep-th/9709001.

[28] А. Гуревич, Л. Питаевский. ЖЭТФ. 1973. Т. 65. С. 590.

[29] H. Flaschka, D. McLaughlin. Progr. Theor. Phys. 1976. V. 55. P. 438.

[30] H. Flaschka, M. Forest, D. McLaughlin. Commun. Pure. Appl. Math. 1980. V. 33. P. 739.

[31] I. Krichever. Funct. Anal. Appl. 1988. V. 22. P. 37; Commun. Math. Phys. 1991. V. 143. P. 415.

[32] Б. Дубровин, С. Новиков. УМН. 1989. Т. 44. № 6. С. 29.

[33] I. Krichever. Commun. Pure Appl. Math. 1994. V. 47. P. 437; The tau function of the universal Whitham hierarchy, matrix models, and topological field theories. Preprint LPTENS-92-18. Paris: Ecole Normale Superiore, 1992.

[34] B. Dubrovin. Geometry of 2d topological field theory. hep-th/9407018; Nucl. Phys. B. 1992. V. 379. P. 627.

[35] H. Itoyama, A. Morozov. Nucl. Phys. B. 1997. V. 491. P. 529; hep-th/9512161.

[36] A. Gorsky, A. Marshakov, A. Mironov, A. Morozov. Nucl. Phys. B. 1998. V. 527. P. 690; hep-th/9802007.

[37] A. Marshakov, M. Martellini, A. Morozov. Phys. Lett. B. 1998. V. 418. P. 294; hep-th $/ 9706050$.

[38] A. Мариаков. ТМФ. 1997. Т. 112. C. 3; hep-th/9702187.

[39] V. Inozemtsev. Commun. Math. Phys. 1989. V. 121. P. 629.

[40] И. Кричевер. Функц. анализ и его прилож. 1980. V. 14. Р. 45.

[41] E. Witten. Branes and the dynamics of QCD. hep-th/9706109.

[42] G. Korchemsky, I. Krichever. Solitons in high-energy QCD. hep-th/9704079.

[43] H. Braden, A. Marshakov, A. Mironov, A. Morozov. Seiberg-Witten theory for a non-trivial compactification from five to four dimensions. hep-th/9812078; The Ruijsenaars-Schneider model in the context of Seiberg-Witten theory. hep-th/9902205; A. Marshakov. Seiberg-Witten curves and integrable systems. hep-th/9903252.

[44] I. Affleck, J. Harvey, E. Witten. Nucl. Phys. B. 1982. V. 206. P. 413.

[45] N. Seiberg, E. Witten. Gauge dynamics and compactification to three dimensions. hep-th/9607163.

3 Теоретическая и математическая физика, т. 121, № 2, 1999 г. 
[46] S. Katz, C. Vafa. Geometric engineering of $N=1$ quantum field theory. hep-th/9611090; O. Aharony, A. Hanany, K. Intriligator, N. Seiberg, M. Strassler. Aspects of $N=1$ supersymmetric gauge theories in three dimensions. hep-th/9703110; C. Vafa. On $N=1$ Yang-Mills in four dimensions. hep-th/9801139.

[47] M. Davies, T. Hollowood, V. Khoze, M. Mattis. Gluino condensate and magnetic monopoles in supersymmetric gluodynamics. hep-th/9905015.

[48] K. Li. Nucl. Phys. B. 1991. V. 354. P. 711; P. 725; T. Eguchi, S.-K. Yang. Mod. Phys. Lett. A. 1990. V. 5. P. 1693; C. Vafa. Mod. Phys. Lett. A. 1991. V. 6. P. 337; E. Witten. Mirror manifolds and topological field theory. hep-th/9112056.

[49] E. Witten. Surv. Diff. Geom. 1991. V. 1. P. 243; R. Dijkgraaf, E. Verlinde, H. Verlinde. Nucl. Phys. B. 1991. V. 352. P. 59.

[50] S. Cecotti, C. Vafa. Phys. Rev. Lett. 1992. V. 68. P. 903; hep-th/9111016; Commun. Math. Phys. 1993. V. 157. P. 139; hep-th/9209085; Commun. Math. Phys. 1993. V. 158. P. 569; hep-th/9211097.

[51] A. Marshakov, A. Mironov, A. Morozov. Phys. Lett. B. 1996. V. 389. P. 43; hep-th/9607109.

[52] E. Witten. Commun. Math. Phys. 1988. V. 118. P. 411.

[53] M. Kontsevich, Yu. Manin. Commun. Math. Phys. 1994. V. 164. P. 525; hep-th/9402147; M. Kontsevich. Enumeration of rational curves via torus actions. hep-th/9405035; Homological algebra of mirror symmetry. alg-geom/9411018; M. Kontsevich, Yu. Manin (with Appendix by $R$. Kaufmann). Quantum cohomology of a product. q-alg/9502009; M. Kontsevich, $Y u$. Manin. Relations between the correlators of the topological sigma-model coupled to gravity. alg-geom/9708024.

[54] A. Givental, B. Kim. Commun. Math. Phys. 1995. V. 168. P. 609; hep-th/9312096; A. Givental. Equivariant Gromov-Witten invariants. alg-geom/9603021; Stationary phase integrals, quantum Toda lattices, flag manifolds, and the mirror conjecture. alg-geom/9612001; A mirror theorem for toric complete intersections. alg-geom/9701016.

[55] A. Marshakov, A. Mironov, A. Morozov. More evidence for the WDVV equations in $N=2$ SUSY Yang-Mills theories. hep-th/9701123.

[56] M. Концевич. Функц. анализ и его прилож. 1991. Т. 25. С. 50.

[57] S. Kharchev, A. Marshakov, A. Mironov, A. Morozov, A. Zabrodin. Phys. Lett. B. 1992. V. 275. P. 311; hep-th/9111037; Nucl. Phys. B. 1992. V. 380. P. 181; hep-th $/ 9201013$; M. Kontsevich. Commun. Math. Phys. 1992. V. 147. P. 1; C. Itzykson, J.-B. Zuber. Int. J. Mod. Phys. A. 1992. V. 7. P. 5661; M. Adler, P. van Moerbeke. Commun. Math. Phys. 1992. V. 147. P. 25.

[58] E. Witten. On the Kontsevich model and other models of two-dimensional gravity. Preprint IASSNS-HEP-91/24. Princeton (New Jersey), USA: Institute of Advanced Study, 1991.

[59] A. Marshakov, A. Mironov, A. Morozov. Phys. Lett. B. 1992. V. 274. P. 280.

[60] S. Kharchev, A. Marshakov, A. Mironov, A. Morozov. Mod. Phys. Lett. A. 1993. V. 8. P. 1047; hep-th/9208046.

[61] S. Kharchev, A. Marshakov. Topological versus non-topological theories and $p-q$-duality in $c<1$ 2D gravity models. In: String Theory, Quantum Gravity and the Unification of Fundamental Interactions. Eds. M. Bianchi et al. Singapore: World Scientific, 1993. P. 331; hep-th/9210072; Int. J. Mod. Phys. A. 1995. V. 10. P. 1219; hep-th/9303100.

[62] S. Kharchev. Kadomtsev-Petviashvili hierarchy and generalized Kontsevich model. Preprint ITEP/TH-78/97; hep-th/9810091.

[63] J. Ambjørn, B. Durhuus, J. Fröhlich. Nucl. Phys. B. 1985. V. 257 [FS14]. P. 433; F. David. Nucl. Phys. B. 1985. V. 257 [FS14]. P. 45; V. Kazakov. Phys. Lett. B. 1985. V. 150. P. 282; V. Kazakov, I. Kostov, A. Migdal. Phys. Lett. B. 1985. V. 157. P. 295. 
[64] V. Kazakov. Mod. Phys. Lett. A. 1989. V. 4. P. 2125; E. Brézin, V. Kazakov. Phys. Lett. B. 1990. V. 236. P. 144; M. Douglas, S. Shenker. Nucl. Phys. B. 1990. V. 335. P. 635; D. Gross, A. Migdal. Phys. Rev. Lett. 1990. V. 64. P. 127.

[65] M. Fukuma, H. Kawai, R. Nakayama. Int. J. Mod. Phys. A. 1991. V. 6. P. 1385; R. Dijkgraaf, E. Verlinde, H. Verlinde. Nucl. Phys. B. 1991. V. 348. P. 435.

[66] T. Eguchi, K. Hori, C. Xiong. Int. J. Mod. Phys. A. 1997. V. 12. P. 1743; hep-th/9605225; Phys. Lett. B. 1997. V. 402. P. 71; hep-th/9703086; T. Eguchi, M. Jinzenji, C. Xiong. Nucl. Phys. B. 1998. V. 510. P. 608; hep-th/9709152; T. Eguchi, C. Xiong. Quantum cohomology at higher genus: topological recursion relations and Virasoro conditions. hep-th/9801010; E. Getzler. Intersection theory on $\overline{\mathcal{M}}_{1,4}$ and elliptic Gromov-Witten invariants. alg-geom/9612004; Topological recursion relations in genus 2. math/9801003; E. Getzler, R. Pandharipande. Frobenius manifolds and Virasoro constraints. math/9805114; B. Dubrovin, Y. Zhang. Virasoro constraints and the Chern classes of the Hodge bundle. math/9808048.

[67] A. Gerasimov, A. Marshakov, A. Mironov, A. Morozov, A. Orlov. Nucl. Phys. B. 1991. V. 357. P. 565.

[68] S. Kharchev, A. Marshakov, A. Mironov, A. Orlov, A. Zabrodin. Nucl. Phys. B. 1991. V. 366. P. 569.

[69] M. Douglas. Phys. Lett. B. 1990. V. 238. P. 176.

[70] I. Krichever. On Heisenberg relations for the ordinary linear differential operators. Preprint. Zuerich: ETH, 1990; С.П. Новиков. Функц. анализ и его прилож. 1990. Т. 24. С. 43; G. Moore. Commun. Math. Phys. 1990. V. 133. P. 261; G. Moore. Matrix models of 2D gravity and isomonodromic deformations. Preprint Yale University, 1990; R. Schimmrigk. Phys. Rev. Lett. 1990. V. 65. P. 2483; P. Grinevich, S. Novikov. solv-int/9501002.

[71] A. Marshakov, A. Mironov. Seiberg-Witten systems and Whitham hierarchies: a short review. Preprint FIAN/TD-04/98, ITEP/TH-51/98; hep-th/9809196.

[72] M. Matone. Phys. Lett. B. 1995. V. 357. P. 342; hep-th/9506102; Phys. Rev. D. 1996. V. 53. P. 7354; hep-th/9506181; J. Sonnenschein, S. Theisen, S. Yankielowicz. On the relation between the holomorphic prepotential and the quantum moduli in SUSY gauge theories. hep-th/9510129; T. Eguchi, S. K. Yang. Mod. Phys. Lett. A. 1996. V. 11. P. 131; hep-th/9510183; P. Howe, P. West. Nucl. Phys. B. 1997. V. 486. P. 425; hep-th/9607239.

[73] A. Losev, N. Nekrasov, S. Shatashvili. Issues in topological gauge theoires. hep-th/9711108.

[74] G. Moore, E. Witten. Integration over the u-plane in Donaldson theory. hep-th/9709193; G. Moore, M. Mariño. Integration over the Coulomb branch in $N=2$ gauge theory. hep-th/9712062; The Donaldson-Witten function for gauge groups of rank larger then one. hep-th/9802185.

Поступила в редакцию 14.V.1999 г. 